\title{
SN 2012fr: Ultraviolet, Optical, and Near-infrared Light Curves of a Type Ia Supernova Observed within a Day of Explosion*
}

\author{
Carlos Contreras $^{1,2}$ (10), M. M. Phillips ${ }^{2}$ (1) , Christopher R. Burns $^{3}$ (1) Anthony L. Piro $^{3}$ (1) B. J. Shappee ${ }^{3}$,
} Maximilian D. Stritzinger ${ }^{1}$ (i) , C. Baltay ${ }^{4}$, Peter J. Brown ${ }^{5}$ (i) , Emmanuel Conseil ${ }^{6}$, Alain Klotz ${ }^{7}$, Peter E. Nugent ${ }^{8,9}$ (i), Damien Turpin $^{7}$ (1) Stu Parker ${ }^{10,24}$, D. Rabinowitz ${ }^{4}$, Eric Y. Hsiao ${ }^{1,2,11}$ (1) , Nidia Morrell ${ }^{2}$ (1) , Abdo Campillay ${ }^{2,12}$, Sergio Castellón ${ }^{2}$, Carlos Corco $^{2,13}$, Consuelo González ${ }^{2}$, Kevin Krisciunas ${ }^{5}$ (1) , Jacqueline Serón ${ }^{2,14}$, Brad E. Tucker ${ }^{15,16}$ (1) , E. S. Walker ${ }^{4,17}$,

E. Baron ${ }^{18}(1)$, C. Cain ${ }^{18}$, Michael J. Childress ${ }^{15,16,19}$, Gastón Folatelli ${ }^{20}$, Wendy L. Freedman ${ }^{3,21}$, Mario Hamuy ${ }^{22}$, P. Hoeflich ${ }^{11}$ (i), S. E. Persson ${ }^{3}$, Richard Scalzo ${ }^{15,16,23}$, Brian Schmidt ${ }^{15}$ (i), and Nicholas B. Suntzeff ${ }^{5}$

${ }^{1}$ Department of Physics and Astronomy, Aarhus University, Ny Munkegade 120, DK-8000 Aarhus C, Denmark

${ }^{2}$ Las Campanas Observatory, Carnegie Observatories, Casilla 601, La Serena, Chile; carlos.astro@gmail.com

${ }^{3}$ Observatories of the Carnegie Institution for Science, 813 Santa Barbara Street, Pasadena, CA 91101, USA

${ }^{4}$ Physics Department, Yale University, 217 Prospect Street, New Haven, CT 06511-8499, USA

${ }^{5}$ George P. and Cynthia Woods Mitchell Institute for Fundamental Physics and Astronomy, Department of Physics and Astronomy, Texas A\&M University, College Station, TX 77843, USA

${ }^{6}$ Association Française des Observateurs d'Étoiles Variables (AFOEV), 11, rue de l'université F-67000 Strasbourg, France

${ }^{7}$ Université de Toulouse, UPS-OMP, IRAP, 14, avenue Edouard Belin, F-31400 Toulouse, France

${ }^{8}$ Lawrence Berkeley National Laboratory, Department of Physics, 1 Cyclotron Road, Berkeley, CA 94720, USA

${ }^{9}$ Astronomy Department, University of California at Berkeley, Berkeley, CA 94720, USA

${ }^{0}$ Backyard Observatory Supernova Search (BOSS), Parkdale Observatory, New Zealand

${ }^{11}$ Department of Physics, Florida State University, Tallahassee, FL 32306, USA
Departamento de Física y Astronomía, Universidad de La Serena, Av. Cisternas 1200, La Serena, Chile

${ }^{13}$ SOAR Telescope, Casilla 603, La Serena, Chile

${ }^{14}$ Cerro Tololo Interamerican Observatory, Casilla 603, La Serena, Chile

15 Research School of Astronomy and Astrophysics, Australian National University, Canberra, ACT 2611, Australia

${ }^{16}$ ARC Centre of Excellence for All-sky Astrophysics (CAASTRO), ANU, Canberra, Australia

${ }^{17}$ Qriously Corporation, 1 Hartwick Street, London EC1R4RB, UK

${ }^{18}$ Homer L. Dodge Department of Physics and Astronomy, 440 W. Brooks Street, Rm 100, Norman, OK 73019, USA

${ }^{19}$ School of Physics and Astronomy, University of Southampton, Southampton, SO17 1BJ, UK

${ }^{20}$ Facultad de Ciencias Astronómicas y Geofísicas, Universidad Nacional de La Plata, Instituto de Astrofísica de La Plata (IALP), CONICET, Paseo del Bosque S/N, B1900FWA La Plata, Argentina

${ }^{21}$ Department of Astronomy and Astrophysics, University of Chicago, 5640 South Ellis Avenue, Chicago, IL 60637, USA

${ }^{22}$ Departamento de Astronomía, Universidad de Chile, Casilla 36-D, Santiago, Chile

23 Centre for Translational Data Science, University of Sydney, Darlington, NSW 2008, Australia

Received 2018 February 21; revised 2018 March 26; accepted 2018 March 29; published 2018 May 18

\begin{abstract}
We present detailed ultraviolet, optical, and near-infrared light curves of the Type Ia supernova (SN) 2012fr, which exploded in the Fornax cluster member NGC 1365. These precise high-cadence light curves provide a dense coverage of the flux evolution from -12 to +140 days with respect to the epoch of $B$-band maximum $\left(t_{B_{\max }}\right)$. Supplementary imaging at the earliest epochs reveals an initial slow and nearly linear rise in luminosity with a duration of $\sim 2.5$ days, followed by a faster rising phase that is well reproduced by an explosion model with a moderate amount of ${ }^{56} \mathrm{Ni}$ mixing in the ejecta. From our analysis of the light curves, we conclude that: (i) the explosion occurred $<22 \mathrm{hr}$ before the first detection of the supernova, (ii) the rise time to peak bolometric $(\lambda>1800 \AA$ ) luminosity was $16.5 \pm 0.6$ days, (iii) the supernova suffered little or no host-galaxy dust reddening, (iv) the peak luminosity in both the optical and near-infrared was consistent with the bright end of normal Type Ia diversity, and (v) $0.60 \pm 0.15 M_{\odot}$ of ${ }^{56} \mathrm{Ni}$ was synthesized in the explosion. Despite its normal luminosity, SN 2012fr displayed unusually prevalent high-velocity Ca II and Si II absorption features, and a nearly constant photospheric velocity of the Si II $\lambda 6355$ line at $\sim 12,000 \mathrm{~km} \mathrm{~s}^{-1}$ that began $\sim 5$ days before $t_{B_{\max }}$. We also highlight some of the other peculiarities in the early phase photometry and the spectral evolution. SN $2012 \mathrm{fr}$ also adds to a growing number of Type Ia supernovae that are hosted by galaxies with direct Cepheid distance measurements.
\end{abstract}

Key words: supernovae: general - supernovae: individual (SN 2012fr)

\section{Introduction}

Type Ia supernovae (SNe Ia) are the major producers of iron in the Galaxy (Tinsley 1979) and, therefore, they are intimately tied

\footnotetext{
* This paper includes data gathered with the $6.5 \mathrm{~m}$ Magellan Baade Telescope, located at Las Campanas Observatory, Chile.

24 http://bosssupernova.com/
}

to its chemical evolution (e.g., see McWilliam 1997). The fact that they are among the brightest and most homogenous of the supernovae ( $\mathrm{SNe}$ ) has allowed them to be used with great success as distance indicators to measure the expansion history of the universe (e.g., see Betoule et al. 2014). Nevertheless, progress has remained disappointingly slow in identifying the progenitor systems of these objects and understanding the details of their explosion mechanisms. More than 50 years ago, Hoyle \& Fowler 




Figure 1. Swope telescope color image of NGC 1365 with local sequence stars and SN $2012 \mathrm{fr}$ indicated.

(1960) recognized that SNe Ia were thermonuclear disruptions of white dwarfs in binary systems. However, there is still considerable debate as to whether the companion is a main-sequence or giant star (single-degenerate model), or is instead another white dwarf (double-degenerate model). The collision of two white dwarfs (as opposed to their merger) has recently garnered interest as a third possible way of producing SNe Ia (Raskin et al. 2009; Rosswog et al. 2009; Thompson 2011; Kushnir et al. 2013). As to the explosion mechanism, the deflagration (subsonic burning) of a Chandrasekhar mass white dwarf that at some point transitions to a supersonic detonation (Khokhlov 1991) provides the best match to the observational properties of SNe Ia (Höflich \& Khokhlov 1996; Höflich et al. 1996; Hoeflich et al. 2017), but the details of how or why this occurs are still a mystery. Meanwhile, detonations of subChandrasekhar-mass white dwarfs also have many attractive properties for explaining SNe Ia (Sim et al. 2010; Shen et al. 2018). These detonations can be triggered in a number of ways, including the double detonation mechanism (e.g., Woosley \& Weaver 1994) from the accretion of a helium shell (e.g., Fink et al. 2010), a detonation in an accretion stream (Guillochon et al. 2010; Dan et al. 2012) in a violent merger involving a massive WD (Pakmor et al. 2012), or even in a more long-term evolution of a merger remnant (Shen et al. 2012).

Howell (2011) emphasized that large samples of SNe Ia help to improve our understanding of the progenitors and explosion mechanisms. For example, measurements of the relative rates in different types of galaxies can be used to infer the delay time distribution, which in turn can serve as a discriminant between different progenitor scenarios (Totani et al. 2008; Maoz et al. 2010). The high precision photometry and spectroscopy of individual SNe Ia also provides an insight into the progenitors and the physics of the explosion mechanism (e.g., see Höflich et al. 2010; Hsiao et al. 2013). Photometric observations of $\mathrm{SNe}$ Ia at the very earliest epochs following an explosion can be used to provide important constraints on the initial radius of the primary star (Piro et al. 2010; Bloom et al. 2012), the size of its companion star (Kasen 2010; Hosseinzadeh et al. 2017), and the distribution of ${ }^{56} \mathrm{Ni}$ in the outermost ejecta and/or the possible presence of circumstellar material (Piro \& Morozova 2016).

SN 2012fr was discovered by the TAROT (Télescopes à Action Rapide pour les Objets Transitoires) collaboration in images taken with their robotic telescope at the La Silla Observatory, Chile (Klotz 2012). With J2000.0 coordinates of $\alpha=03^{\mathrm{h}} 33^{\mathrm{m}} 35^{\mathrm{s}} .99$ and $\delta=-36^{\circ} 07^{\prime} 37^{\prime \prime}$, the transient was located $3^{\prime \prime}$ west and $52^{\prime \prime}$ north from the center of the SBb host-galaxy NGC 1365 (see Figure 1). Within a day and a half of the discovery, SN 2012fr was spectroscopically classified as a young, normal SN Ia, which was caught well before maximum light (Childress et al. 2012). Childress et al. (2013) analyzed an extensive set of optical spectral data of SN 2012fr, and documented the presence of high-velocity $\mathrm{Si}$ II and $\mathrm{Ca}$ II absorption components in the first spectrum obtained 


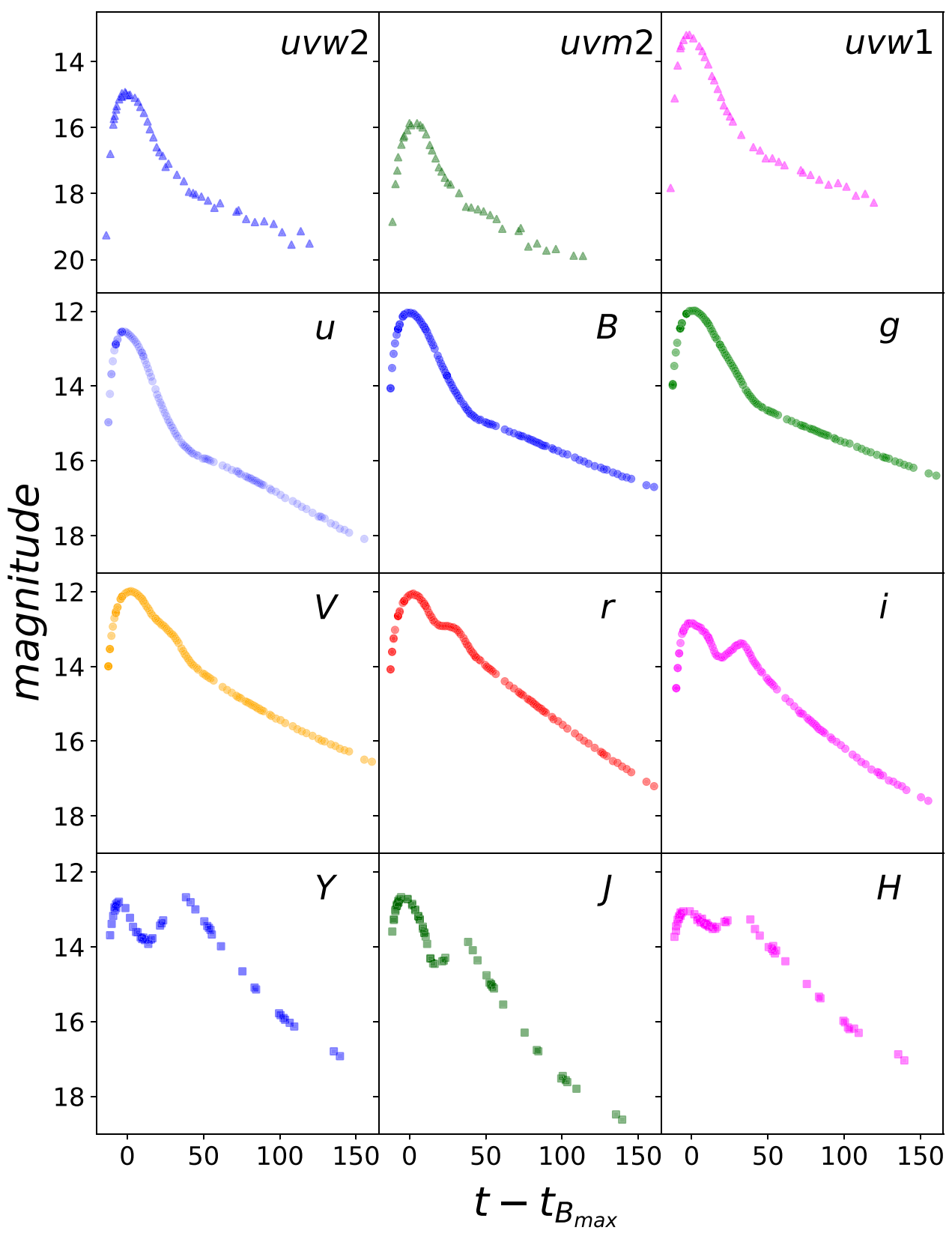

Figure 2. UV, optical, and NIR light curves of SN 2012fr. For the Swift UV bands, the error bars are omitted for clarity. For the optical and NIR bands, the error bars are smaller in size than the symbols.

more than 14 days before $t_{B_{\max }}$, which persisted for the two weeks leading up to maximum. Although the presence of such highvelocity features (HVFs) at early epochs is common, it is relatively unusual for them to remain visible at maximum light (Mazzali et al. 2005). The evolution of the photospheric velocity as inferred from the Si II $\lambda 6355$ absorption was also unusually flat. Nevertheless, the overall spectral evolution of SN 2012fr was that of a normal SN Ia. Maund et al. (2013) published spectropolarimetry at four epochs between 11 days before and 24 days after $t_{B_{\max }}$. They found that although the continuum polarization of the $\mathrm{SN}$ was low $(<0.01 \%)$ throughout this period, the Si II and Ca II HVFs were highly polarized at the earliest epoch. Zhang et al. (2014) also published extensive ultraviolet and optical photometry of SN 2012fr, along with optical spectroscopy, and they conjectured that SN 2012fr might be related to the 1991T-like events but was viewed from a direction where the ejecta presented a clumpy structure. More recently, Graham et al. (2017) presented Las Cumbres Observatory early-time gri and BVRI photometry of SN 2012fr, and Childress et al. (2015) and Graham et al. (2017) published nebular-phase spectra.

Between 2004 and 2009, the Carnegie Supernova Project (CSP-I, Hamuy et al. 2006) carried out precise optical and nearinfrared (NIR) photometry of more than 100 nearby SNe Ia using the facilities at the Las Campanas Observatory (LCO). A second phase of the CSP (CSP-II) was initiated in 2011 to obtain the optical and NIR light curves of $\sim 100$ SNe Ia located further in the Hubble flow at $z \simeq 0.03-0.08$ (M. M. Phillips et al. 2018, in preparation). In addition, CSP-II obtained light curves of a sample of $\sim 100$ more nearby SNe Ia at $z \leqslant 0.04$, for which detailed NIR spectroscopic time-series observations could be made. In this paper we present comprehensive optical and NIR light curves of one of the latter objects, SN $2012 \mathrm{fr}$. 
Table 1

UVOT Photometry of SN 2012fr in the Vega System

\begin{tabular}{|c|c|c|c|c|c|c|}
\hline JD (days) & $u v w 2$ (mag) & JD (days) & uvm2 (mag) & JD (days) & $u v w 1$ (mag) & Phase (days) \\
\hline 2456229.32 & $19.139(186)$ & $\ldots$ & $\ldots$ & 2456229.31 & $17.795(115)$ & -13.78 \\
\hline 2456231.99 & $16.798(077)$ & 2456232.00 & $18.815(118)$ & 2456231.99 & $15.090(058)$ & -11.11 \\
\hline 2456233.93 & $15.936(074)$ & 2456233.94 & $17.676(101)$ & 2456233.92 & $14.096(046)$ & -9.17 \\
\hline 2456235.68 & $15.625(074)$ & 2456235.68 & $16.977(081)$ & 2456235.71 & $13.559(044)$ & -7.42 \\
\hline 2456236.17 & $15.303(064)$ & $\ldots$ & $\ldots$ & 2456236.16 & $13.502(042)$ & -6.93 \\
\hline 2456237.80 & $15.099(063)$ & 2456237.80 & $16.603(070)$ & 2456237.70 & $13.332(044)$ & -5.30 \\
\hline$\ldots$ & $\ldots$ & 2456239.17 & $16.372(072)$ & $\ldots$ & $\ldots$ & -3.93 \\
\hline 2456239.60 & $14.913(075)$ & 2456239.60 & $16.327(108)$ & 2456239.60 & $13.176(044)$ & -3.50 \\
\hline 2456241.64 & $14.878(068)$ & 2456241.64 & $16.091(084)$ & 2456241.64 & $13.165(043)$ & -1.46 \\
\hline 2456242.14 & $14.914(058)$ & $\ldots$ & $\ldots$ & $\ldots$ & $\ldots$ & -0.96 \\
\hline 2456243.13 & $14.980(063)$ & 2456243.11 & $15.919(078)$ & $\ldots$ & $\ldots$ & +0.03 \\
\hline 2456244.90 & $14.958(054)$ & $\ldots$ & $\ldots$ & $\ldots$ & $\ldots$ & +1.80 \\
\hline 2456250.13 & $15.170(072)$ & 2456250.14 & $15.950(073)$ & 2456250.13 & $13.655(045)$ & +7.03 \\
\hline 2456251.70 & $15.351(075)$ & 2456251.70 & $16.021(074)$ & 2456251.69 & $13.839(047)$ & +8.60 \\
\hline 2456254.00 & $15.548(077)$ & 2456254.00 & $16.242(077)$ & 2456253.99 & $14.062(050)$ & +10.90 \\
\hline 2456262.36 & $16.540(088)$ & 2456262.36 & $17.191(095)$ & 2456262.35 & $15.089(067)$ & +19.26 \\
\hline 2456264.15 & $16.692(097)$ & 2456264.15 & $17.296(111)$ & 2456264.15 & $15.329(072)$ & +21.05 \\
\hline 2456268.23 & $17.077(103)$ & 2456268.23 & $17.630(117)$ & 2456268.22 & $15.673(075)$ & +25.13 \\
\hline 2456270.09 & $17.035(104)$ & 2456270.10 & $17.685(124)$ & 2456270.09 & $15.827(078)$ & +26.99 \\
\hline 2456280.18 & $17.578(110)$ & 2456280.19 & $18.356(140)$ & 2456280.18 & $16.822(091)$ & +37.08 \\
\hline 2456287.96 & $17.953(118)$ & 2456287.96 & $18.434(137)$ & 2456287.96 & $16.665(082)$ & +44.86 \\
\hline 2456296.02 & $18.132(131)$ & 2456296.02 & $18.606(165)$ & 2456296.01 & $16.899(090)$ & +52.92 \\
\hline 2456300.22 & $18.345(144)$ & 2456300.23 & $18.726(166)$ & 2456300.22 & $17.005(094)$ & +57.12 \\
\hline 2456303.96 & 18.207(139) & 2456303.97 & $19.020(201)$ & 2456303.96 & $17.109(099)$ & +60.86 \\
\hline 2456314.69 & $18.453(133)$ & 2456314.69 & $19.082(177)$ & 2456314.68 & $17.258(091)$ & +71.59 \\
\hline 2456332.85 & $18.727(166)$ & 2456332.86 & $19.668(288)$ & 2456332.85 & $17.690(116)$ & +89.75 \\
\hline 2456350.74 & $19.386(232)$ & 2456350.75 & $19.815(306)$ & 2456350.74 & $18.016(130)$ & +107.64 \\
\hline 2456356.82 & $19.010(178)$ & 2456356.83 & $19.823(280)$ & 2456356.82 & $17.963(121)$ & +113.72 \\
\hline
\end{tabular}

Note. The values in parentheses are $1 \sigma$ measurement uncertainties and they are given in millimag. The phase is relative to $t_{B_{\max }}=\mathrm{JD} 2456243.1$.

We also analyze the earliest images of the SN, including prediscovery observations obtained by the La Silla Quest survey less than one day after the explosion.

The host-galaxy of SN 2012fr, NGC 1365, is a member of the Fornax cluster and it has numerous published Tully-Fisher and Cepheid distance measurements. In the following, we adopt the Freedman et al. (2001) Cepheid distance modulus of $\mu=31.27 \pm 0.05$ mag because of its internal consistency with the SNooPy analysis tool that we employ in this paper. ${ }^{25}$ The galactic reddening in the direction of NGC 1365 as computed from the Schlafly \& Finkbeiner (2011) recalibration of the Schlegel et al. (1998) infrared dust maps is $E(B-V)_{\mathrm{MW}}=$ $0.018 \pm 0.003$ mag. When adopting a Fitzpatrick (1999) reddening law characterized by $R_{V}=3.1$, this corresponds to $A_{V}=0.056 \pm 0.009 \mathrm{mag}$. As discussed in Section 3.2, an analysis of the optical and NIR light curves indicates that SN 2012fr suffered little or no host-galaxy reddening.

The rest of this paper is organized as follows. Section 2 presents the observational data, consisting of the broadband ultraviolet (UV), optical, and NIR photometry. In Section 3, these observations are analyzed to derive the light-curve parameters, the host-galaxy reddening, an independent estimate of the distance based on the $\mathrm{SN}$ properties, a bolometric light curve, the rise time to maximum, and the mass of the ${ }^{56} \mathrm{Ni}$ produced in the explosion. Section 4 discusses the early light curve and makes an assessment

\footnotetext{
25 Freedman et al.'s (2001) distance modulus for NGC 1365 is consistent within the errors with those obtained more recently by Riess et al. (2016) from Cepheid variables and by Jang et al. (2018) from the tip of the red giant branch method.
}

of the normality of SN 2012fr. Finally, the main conclusions of this study are summarized in Section 5.

\section{Observations}

\subsection{Ultraviolet Photometry}

The imaging of SN 2012fr was performed from space with the Swift UltraViolet Optical Telescope (UVOT). In this paper, we only present the $u v w 2$, $u v m 2$, and $u v w 1$ passbands. According to Brown et al. (2016), the effective wavelengths of these filters (convolved with an $\mathrm{SN}$ Ia spectrum of SN $1992 \mathrm{~A}$, at +5 epoch) are $2010 \AA, 2360 \AA$, and $2890 \AA$, respectively.

The photometry of SN 2012fr was computed from these images following the techniques described in detail by Brown et al. (2009, see their Section 2). In short, a $3^{\prime \prime}$ to $5^{\prime \prime}$ source aperture was used for each image, depending on the signal-tonoise ratio, to measure the counts at the position of the $\mathrm{SN}$. The count rate from the underlying galaxy was then subtracted from these counts using images without the supernova present. The corrected counts were converted to the UVOT photometric system (Poole et al. 2008), while adopting the zero points provided by Breeveld et al. (2011). The measured instrumental magnitudes are corrected for a coincidence loss correction factor that is based on measurements made with a $5^{\prime \prime}$ aperture. In addition, an aperture correction is applied based on an average point-spread function (PSF) in the Swift calibration database.

Covering $\sim 40$ epochs ranging from -13 days to +120 days relative to the epoch of $t_{B_{\max }}$ (see Section 3), the high-cadence UV light curves of SN $2012 \mathrm{fr}$ are plotted in Figure 2. The corresponding photometry is listed in Table 1 . 
Table 2

Photometry of SN 2012fr Local Sequence Stars in the Standard System

\begin{tabular}{|c|c|c|c|c|c|c|c|c|c|c|c|}
\hline Star ID & R.A. & Decl. & $u$ (mag) & $g$ (mag) & $r$ (mag) & $\overline{i \text { (mag) }}$ & $B$ (mag) & $V$ (mag) & $Y(\mathrm{mag})$ & $J$ (mag) & 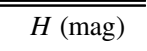 \\
\hline 01 & $03: 33: 55.48$ & $-36: 07: 19.0$ & $16.836(010)$ & $14.087(003)$ & $12.846(002)$ & $12.332(009)$ & $14.748(003)$ & $13.423(002)$ & $\ldots$ & $\ldots$ & $\ldots$ \\
\hline 02 & 03:33:33.67 & $-36: 07: 14.1$ & $15.314(004)$ & $13.879(002)$ & $13.343(002)$ & $13.160(008)$ & $14.293(003)$ & $13.560(002)$ & $12.467(034)$ & $12.167(023)$ & $11.821(035)$ \\
\hline 03 & 03:33:49.55 & $-36: 07: 42.8$ & 15.781(006) & $14.774(003)$ & $14.450(003)$ & $14.370(008)$ & $15.062(004)$ & $14.569(003)$ & $\ldots$ & $\ldots$ & $\ldots$ \\
\hline 04 & 03:33:55.66 & $-36: 08: 38.2$ & 16.881(007) & $15.385(004)$ & $14.833(003)$ & 14.641(009) & $15.803(006)$ & $15.050(004)$ & $\ldots$ & $\ldots$ & $\ldots$ \\
\hline 05 & 03:33:40.67 & $-36: 10: 52.9$ & $16.565(007)$ & $15.368(004)$ & $14.924(004)$ & $14.789(009)$ & $15.732(006)$ & 15.091(005) & $\cdots$ & $\ldots$ & $\ldots$ \\
\hline 06 & 03:33:57.36 & $-36: 09: 24.0$ & $18.750(074)$ & 17.019(011) & $16.429(007)$ & $16.244(014)$ & 17.463(014) & $16.689(014)$ & $\ldots$ & $\ldots$ & $\ldots$ \\
\hline 07 & 03:33:54.96 & $-36: 09: 09.6$ & $18.769(020)$ & $17.017(015)$ & $16.423(010)$ & $16.237(014)$ & $17.487(030)$ & $16.659(013)$ & $\ldots$ & $\ldots$ & $\ldots$ \\
\hline 08 & $03: 33: 46.83$ & $-36: 03: 04.1$ & $15.247(007)$ & $14.249(005)$ & $13.916(005)$ & $13.790(005)$ & $\ldots$ & $\cdots$ & $\ldots$ & $\ldots$ & $\ldots$ \\
\hline 12 & 03:33:16.73 & $-36: 05: 08.9$ & $18.748(001)$ & $16.033(012)$ & $14.789(005)$ & $14.267(010)$ & $\ldots$ & $\ldots$ & $\ldots$ & $\ldots$ & $\ldots$ \\
\hline 13 & $03: 33: 15.45$ & $-36: 03: 47.4$ & $18.102(007)$ & $16.955(014)$ & $16.555(007)$ & $16.417(012)$ & $\ldots$ & $\ldots$ & $\ldots$ & $\ldots$ & $\ldots$ \\
\hline 102 & 03:33:31.07 & $-36: 08: 11.8$ & $\ldots$ & $\ldots$ & $\ldots$ & $\ldots$ & $\ldots$ & $\ldots$ & $14.303(040)$ & $13.933(173)$ & $13.432(030)$ \\
\hline 103 & $03: 33: 45.26$ & $-36: 07: 29.4$ & $\ldots$ & $\ldots$ & $\ldots$ & $\ldots$ & $\ldots$ & $\ldots$ & $15.506(035)$ & $15.011(033)$ & $14.463(061)$ \\
\hline
\end{tabular}

Note. Values in parentheses are $1 \sigma$ measurement uncertainties in millimag. 
Table 3

Optical Photometry of SN 2012fr in the Natural System of the Swope

\begin{tabular}{|c|c|c|c|c|c|c|c|}
\hline JD (days) & Phase (days) & $u$ (mag) & $g$ (mag) & $r$ (mag) & $i$ (mag) & $B$ (mag) & $V$ (mag) \\
\hline 2456230.70 & -12.40 & $14.975(015)$ & 13.952(009) & $14.079(008)$ & $14.576(009)$ & $14.066(027)$ & $13.990(015)$ \\
\hline 2456231.70 & -11.40 & $14.220(017)$ & $13.458(011)$ & $13.609(010)$ & $14.028(011)$ & $13.524(025)$ & $13.528(011)$ \\
\hline 2456232.70 & -10.40 & $13.688(016)$ & 13.098(010) & $13.248(009)$ & 13.646(009) & $13.126(025)$ & $13.178(009)$ \\
\hline 2456233.62 & -9.48 & $13.351(018)$ & $12.841(010)$ & 13.022(009) & $13.369(010)$ & $12.846(026)$ & $12.931(010)$ \\
\hline 2456234.66 & -8.44 & 13.061(019) & $\cdots$ & $12.778(012)$ & $13.136(012)$ & $12.621(026)$ & $12.707(011)$ \\
\hline 2456235.60 & -7.50 & $12.901(017)$ & $12.450(011)$ & $12.647(012)$ & $13.026(013)$ & $12.465(026)$ & $12.569(013)$ \\
\hline 2456236.69 & -6.41 & $12.758(017)$ & $12.312(012)$ & $12.515(012)$ & $12.941(013)$ & $12.333(028)$ & $12.417(013)$ \\
\hline 2456238.72 & -4.38 & $12.594(017)$ & $\cdots$ & $12.298(010)$ & $12.867(013)$ & 12.113(030) & $12.196(015)$ \\
\hline 2456239.67 & -3.43 & $12.564(015)$ & 12.058(009) & $12.248(009)$ & $12.843(010)$ & $12.109(027)$ & $12.128(011)$ \\
\hline 2456241.67 & -1.43 & $12.559(015)$ & $12.001(015)$ & $12.149(009)$ & $12.842(009)$ & $12.021(025)$ & $12.051(010)$ \\
\hline 2456242.64 & -0.46 & $12.582(016)$ & 11.994(010) & $12.107(008)$ & $12.851(010)$ & $12.051(027)$ & $12.016(011)$ \\
\hline 2456243.63 & +0.53 & $12.621(019)$ & $11.985(012)$ & 12.084(012) & $12.888(017)$ & $12.018(030)$ & $12.002(012)$ \\
\hline 2456244.65 & +1.55 & $12.649(015)$ & $11.988(011)$ & 12.075(009) & $12.913(011)$ & $12.060(028)$ & $11.994(010)$ \\
\hline 2456245.61 & +2.51 & $12.687(019)$ & $12.004(013)$ & $12.061(015)$ & $12.920(018)$ & $12.057(030)$ & $11.981(016)$ \\
\hline 2456246.63 & +3.53 & $12.734(017)$ & $12.010(011)$ & $12.093(014)$ & $12.946(014)$ & $12.102(028)$ & $12.000(013)$ \\
\hline 2456247.65 & +4.55 & $12.776(018)$ & $12.044(012)$ & $12.096(011)$ & $12.966(015)$ & $12.140(029)$ & $12.015(012)$ \\
\hline 2456248.62 & +5.52 & $12.833(015)$ & $12.072(011)$ & $12.116(010)$ & $13.043(013)$ & $12.168(029)$ & $12.035(010)$ \\
\hline 2456249.66 & +6.56 & $12.890(016)$ & $12.109(011)$ & $12.148(009)$ & $13.060(012)$ & $12.237(029)$ & $12.060(011)$ \\
\hline 2456250.67 & +7.57 & $12.967(021)$ & $12.153(014)$ & $12.227(014)$ & $13.094(016)$ & $12.294(030)$ & $12.102(017)$ \\
\hline 2456251.63 & +8.53 & $13.053(019)$ & $12.213(015)$ & $12.253(014)$ & $13.164(013)$ & $12.342(033)$ & $12.141(015)$ \\
\hline 2456252.68 & +9.58 & $13.125(016)$ & $12.269(009)$ & $12.322(008)$ & $13.214(010)$ & $12.416(025)$ & $12.193(010)$ \\
\hline 2456253.61 & +10.51 & $13.222(016)$ & $12.324(009)$ & $12.387(008)$ & $13.310(009)$ & $12.480(025)$ & $12.265(011)$ \\
\hline 2456254.64 & +11.54 & $13.329(017)$ & $12.370(011)$ & $12.456(010)$ & $13.378(011)$ & $12.544(026)$ & $12.311(010)$ \\
\hline 2456255.67 & +12.57 & $13.434(018)$ & $12.464(013)$ & $12.554(013)$ & $13.475(014)$ & $12.646(026)$ & $12.390(013)$ \\
\hline 2456256.60 & +13.50 & $13.537(018)$ & $12.528(013)$ & $12.623(013)$ & $13.571(014)$ & $12.745(030)$ & $12.433(015)$ \\
\hline 2456257.60 & +14.50 & $13.655(018)$ & $12.612(012)$ & $12.682(013)$ & $13.658(013)$ & $12.835(028)$ & $12.497(012)$ \\
\hline 2456258.62 & +15.52 & $13.765(018)$ & $12.692(011)$ & $12.755(011)$ & $13.712(012)$ & $12.891(028)$ & $12.582(013)$ \\
\hline 2456259.59 & +16.49 & $13.884(018)$ & $12.749(010)$ & $12.808(009)$ & 13.732(012) & $13.000(030)$ & $12.629(011)$ \\
\hline 2456261.62 & +18.52 & $14.096(016)$ & $12.890(009)$ & $12.874(009)$ & $13.747(010)$ & 13.174(027) & $12.716(010)$ \\
\hline 2456262.65 & +19.55 & $14.240(016)$ & $12.959(012)$ & $12.901(012)$ & $13.759(013)$ & $13.268(027)$ & $12.772(012)$ \\
\hline 2456263.65 & +20.55 & $14.334(015)$ & 13.022(009) & $12.916(009)$ & $13.714(011)$ & 13.392(028) & $12.791(011)$ \\
\hline 2456264.69 & +21.59 & $14.445(016)$ & $13.114(010)$ & $12.920(009)$ & 13.683(011) & 13.464(027) & $12.837(011)$ \\
\hline 2456265.63 & +22.53 & $14.523(017)$ & 13.171(011) & $12.921(011)$ & $13.648(013)$ & $13.543(027)$ & $12.885(012)$ \\
\hline 2456266.64 & +23.54 & $14.630(016)$ & $13.234(011)$ & $12.922(010)$ & 13.632(011) & $13.642(028)$ & $12.896(011)$ \\
\hline 2456267.65 & +24.55 & $14.726(017)$ & $13.308(012)$ & $12.915(013)$ & $13.579(013)$ & $13.762(038)$ & $12.935(013)$ \\
\hline 2456268.74 & +25.64 & $14.816(015)$ & 13.382(009) & $12.926(008)$ & 13.554(009) & 13.817(027) & $12.985(010)$ \\
\hline 2456269.78 & +26.68 & $14.912(017)$ & 13.451(010) & $12.930(010)$ & $13.518(013)$ & $13.900(029)$ & $13.028(013)$ \\
\hline 2456270.74 & +27.64 & $14.985(015)$ & $13.518(010)$ & $12.946(010)$ & $13.470(012)$ & $13.982(027)$ & $13.065(011)$ \\
\hline 2456271.74 & +28.64 & $15.065(017)$ & $13.590(012)$ & $12.950(011)$ & $13.429(013)$ & $14.080(030)$ & $13.116(013)$ \\
\hline 2456272.74 & +29.64 & $15.125(015)$ & 13.674(011) & $12.983(010)$ & $13.421(011)$ & $14.105(029)$ & $13.141(012)$ \\
\hline 2456273.71 & +30.61 & $15.209(016)$ & 13.731(011) & $12.995(011)$ & $13.411(012)$ & $14.165(028)$ & $13.186(012)$ \\
\hline 2456274.69 & +31.59 & $15.284(016)$ & 13.806(011) & 13.034(010) & 13.374(011) & $14.258(028)$ & $13.250(012)$ \\
\hline 2456275.72 & +32.62 & $15.341(017)$ & $13.880(011)$ & 13.082(012) & $13.375(014)$ & $14.340(030)$ & $13.305(014)$ \\
\hline 2456276.75 & +33.65 & $15.409(017)$ & $13.966(012)$ & 13.141(010) & $13.388(014)$ & 14.401(030) & $13.371(013)$ \\
\hline 2456278.61 & +35.51 & $15.520(016)$ & 14.104(011) & $13.244(011)$ & $13.477(012)$ & $14.486(028)$ & $13.515(011)$ \\
\hline 2456279.74 & +36.64 & $15.581(017)$ & $14.165(011)$ & $13.334(010)$ & $13.546(013)$ & $14.545(036)$ & $13.587(013)$ \\
\hline 2456280.73 & +37.63 & $15.617(016)$ & $14.229(010)$ & $13.409(010)$ & $13.633(010)$ & $14.608(026)$ & $13.667(012)$ \\
\hline 2456281.67 & +38.57 & $15.648(015)$ & $14.275(010)$ & 13.456(009) & $13.694(010)$ & $14.646(025)$ & $13.716(011)$ \\
\hline 2456282.76 & +39.66 & $15.683(016)$ & $14.330(010)$ & $13.539(008)$ & $13.789(009)$ & $14.730(026)$ & $13.784(009)$ \\
\hline 2456283.68 & +40.58 & $15.736(016)$ & $14.384(011)$ & $13.595(010)$ & $13.850(012)$ & $14.747(026)$ & $13.831(011)$ \\
\hline 2456284.72 & +41.62 & $15.754(016)$ & $14.423(009)$ & $13.645(008)$ & $13.910(010)$ & $14.784(025)$ & $13.897(009)$ \\
\hline 2456285.65 & +42.55 & $15.806(016)$ & $14.466(011)$ & $13.698(012)$ & $13.960(011)$ & $14.828(027)$ & $13.938(013)$ \\
\hline 2456286.65 & +43.55 & $15.820(017)$ & 14.492(011) & $13.745(010)$ & $14.009(012)$ & $14.859(027)$ & $13.973(012)$ \\
\hline 2456288.73 & +45.63 & $15.860(017)$ & $14.548(010)$ & 13.816(009) & $14.127(011)$ & $14.908(026)$ & $14.041(011)$ \\
\hline 2456289.60 & +46.50 & $15.877(017)$ & $14.574(012)$ & $13.846(011)$ & $14.158(013)$ & $14.906(038)$ & $14.079(012)$ \\
\hline 2456292.65 & +49.55 & $15.950(016)$ & $14.642(011)$ & $13.960(010)$ & $14.299(012)$ & $14.963(027)$ & $14.184(011)$ \\
\hline 2456293.69 & +50.59 & $15.944(016)$ & $14.656(012)$ & $14.005(011)$ & $14.348(015)$ & $14.969(026)$ & $14.205(011)$ \\
\hline 2456294.68 & +51.58 & $15.958(015)$ & $14.687(009)$ & $14.040(009)$ & $14.407(011)$ & $14.999(026)$ & $14.245(011)$ \\
\hline 2456295.66 & +52.56 & $15.963(016)$ & $14.692(009)$ & $14.070(010)$ & $14.435(012)$ & $15.006(026)$ & $14.266(011)$ \\
\hline 2456296.60 & +53.50 & $15.988(016)$ & $14.718(010)$ & $14.088(011)$ & $14.476(012)$ & $15.016(025)$ & $14.294(013)$ \\
\hline 2456297.67 & +54.57 & $16.010(015)$ & $14.738(009)$ & $14.136(008)$ & $14.518(008)$ & $15.039(025)$ & $14.319(009)$ \\
\hline 2456299.69 & +56.59 & $16.041(016)$ & 14.774(009) & $14.207(009)$ & $14.608(008)$ & $15.066(024)$ & $14.374(009)$ \\
\hline 2456305.60 & +62.50 & 16.134(016) & 14.881(010) & $14.398(009)$ & $14.840(010)$ & $15.157(025)$ & $14.547(011)$ \\
\hline 2456308.55 & +65.45 & $16.188(017)$ & $14.928(013)$ & $14.496(014)$ & $14.940(015)$ & $15.205(027)$ & $14.623(013)$ \\
\hline
\end{tabular}


Table 3

(Continued)

\begin{tabular}{|c|c|c|c|c|c|c|c|}
\hline JD (days) & Phase (days) & $u$ (mag) & $g(\mathrm{mag})$ & $r(\mathrm{mag})$ & $i$ (mag) & $B$ (mag) & $V(\mathrm{mag})$ \\
\hline 2456311.61 & +68.51 & $16.253(017)$ & $14.982(011)$ & $14.588(011)$ & $15.059(014)$ & $15.250(028)$ & $14.700(012)$ \\
\hline 2456314.59 & +71.49 & $16.289(016)$ & $15.039(011)$ & $14.682(010)$ & $15.176(011)$ & $15.303(027)$ & $14.781(011)$ \\
\hline 2456315.63 & +72.53 & $16.297(019)$ & $15.057(010)$ & $14.718(010)$ & $15.239(021)$ & $15.335(028)$ & $14.815(012)$ \\
\hline 2456316.56 & +73.46 & $16.351(017)$ & $15.066(010)$ & $14.743(010)$ & $15.247(011)$ & $15.329(028)$ & $14.825(011)$ \\
\hline 2456317.58 & +74.48 & $16.369(017)$ & $15.085(012)$ & $14.769(011)$ & $15.265(014)$ & $15.343(027)$ & $14.850(012)$ \\
\hline 2456320.57 & +77.47 & $16.420(018)$ & $15.131(009)$ & $14.866(009)$ & $15.367(011)$ & $15.408(028)$ & $14.929(011)$ \\
\hline 2456321.56 & +78.46 & $16.439(017)$ & $15.152(010)$ & $14.894(009)$ & $15.418(011)$ & $15.426(028)$ & $14.947(011)$ \\
\hline 2456322.59 & +79.49 & $16.470(016)$ & $15.153(011)$ & $14.920(011)$ & $15.432(013)$ & $15.450(028)$ & $14.968(012)$ \\
\hline 2456323.62 & +80.52 & $16.472(016)$ & $15.178(010)$ & $14.947(011)$ & $15.480(014)$ & $15.439(027)$ & $14.986(013)$ \\
\hline 2456324.57 & +81.47 & $16.492(016)$ & $15.198(010)$ & $14.997(009)$ & $15.515(011)$ & $15.474(027)$ & $15.022(012)$ \\
\hline 2456325.56 & +82.46 & $16.532(016)$ & $15.214(011)$ & $15.023(011)$ & $15.531(014)$ & $15.475(028)$ & $15.033(012)$ \\
\hline 2456326.59 & +83.49 & $16.540(016)$ & $15.231(011)$ & $15.057(010)$ & $15.574(011)$ & $15.512(028)$ & $15.055(011)$ \\
\hline 2456327.56 & +84.46 & $16.544(017)$ & $15.255(010)$ & $15.093(009)$ & $15.626(011)$ & $15.502(028)$ & $15.083(011)$ \\
\hline 2456328.57 & +85.47 & $16.588(017)$ & $15.269(009)$ & $15.117(009)$ & $15.663(013)$ & $15.534(027)$ & $15.119(011)$ \\
\hline 2456329.56 & +86.46 & $16.602(016)$ & $15.279(011)$ & $15.134(011)$ & $15.681(014)$ & $15.568(028)$ & $15.120(013)$ \\
\hline 2456330.55 & +87.45 & $16.620(017)$ & $15.296(010)$ & $15.188(010)$ & $15.709(014)$ & $15.616(030)$ & $15.165(013)$ \\
\hline 2456331.53 & +88.43 & $16.651(016)$ & $15.313(010)$ & $15.206(010)$ & $\ldots$ & $15.596(027)$ & $15.175(011)$ \\
\hline 2456332.52 & +89.42 & $16.663(016)$ & $15.324(010)$ & $15.238(009)$ & $15.779(013)$ & $15.603(027)$ & $15.192(011)$ \\
\hline 2456336.56 & +93.46 & $16.758(016)$ & $15.389(010)$ & $15.353(010)$ & $15.895(013)$ & $15.663(028)$ & $15.288(011)$ \\
\hline 2456337.57 & +94.47 & $16.794(017)$ & $15.420(009)$ & $15.409(009)$ & $15.941(013)$ & $15.700(028)$ & $15.320(011)$ \\
\hline 2456340.57 & +97.47 & $16.840(016)$ & $15.464(011)$ & $15.466(012)$ & $16.012(015)$ & $15.725(028)$ & $15.390(012)$ \\
\hline 2456343.57 & +100.47 & $16.922(017)$ & $15.507(009)$ & $15.559(010)$ & $16.099(013)$ & $15.806(028)$ & $15.432(011)$ \\
\hline 2456346.60 & +103.50 & $17.012(027)$ & $15.535(012)$ & $15.657(011)$ & $16.193(017)$ & $15.839(029)$ & $15.510(014)$ \\
\hline 2456351.58 & +108.48 & $17.088(018)$ & $15.627(010)$ & $15.793(010)$ & $16.349(013)$ & $15.904(026)$ & $15.593(011)$ \\
\hline 2456354.55 & +111.45 & $17.163(016)$ & $15.676(010)$ & $15.897(011)$ & $16.430(017)$ & $15.975(029)$ & $15.676(013)$ \\
\hline 2456357.55 & +114.45 & $17.242(017)$ & $15.736(009)$ & $15.990(011)$ & $16.543(014)$ & $16.005(027)$ & $15.731(011)$ \\
\hline 2456360.51 & +117.41 & $17.300(016)$ & $15.772(009)$ & $16.058(011)$ & $16.605(016)$ & $16.066(027)$ & $15.780(011)$ \\
\hline 2456364.53 & +121.43 & $17.396(018)$ & $15.838(010)$ & $16.177(010)$ & $16.740(015)$ & $16.142(027)$ & $15.850(011)$ \\
\hline 2456366.51 & +123.41 & $17.449(017)$ & $15.870(009)$ & $16.227(011)$ & $16.748(016)$ & $16.166(027)$ & $15.897(011)$ \\
\hline 2456368.51 & +125.41 & $17.506(017)$ & $15.894(009)$ & $16.274(013)$ & $16.799(017)$ & $16.176(027)$ & $15.932(011)$ \\
\hline 2456369.51 & +126.41 & $17.500(017)$ & $15.909(010)$ & $16.305(013)$ & $16.822(017)$ & $\ldots$ & $\ldots$ \\
\hline 2456370.55 & +127.45 & $17.524(032)$ & $15.919(009)$ & $16.345(012)$ & $16.881(015)$ & $16.227(026)$ & $15.978(010)$ \\
\hline 2456372.50 & +129.40 & $17.551(018)$ & $15.942(014)$ & $16.395(012)$ & $16.904(016)$ & $16.237(026)$ & $16.000(011)$ \\
\hline 2456376.50 & +133.40 & $17.683(022)$ & $16.010(009)$ & $16.518(013)$ & $17.021(016)$ & $16.309(026)$ & $16.081(010)$ \\
\hline 2456379.52 & +136.42 & $17.724(025)$ & $16.049(011)$ & $16.575(013)$ & $17.051(018)$ & $16.354(027)$ & $16.124(012)$ \\
\hline 2456382.49 & +139.39 & $\ldots$ & 16.102(009) & $16.666(012)$ & $17.142(016)$ & $16.412(026)$ & 16.194(011) \\
\hline 2456385.49 & +142.39 & 17.852(019) & $16.141(009)$ & $16.748(012)$ & $17.206(016)$ & $16.435(026)$ & $16.237(011)$ \\
\hline 2456388.48 & +145.38 & $17.933(021)$ & $16.181(010)$ & $16.830(013)$ & $17.279(018)$ & $16.480(030)$ & $16.270(012)$ \\
\hline 2456398.48 & +155.38 & $18.100(030)$ & $16.336(015)$ & $17.085(016)$ & $17.474(020)$ & $16.636(029)$ & $16.484(018)$ \\
\hline 2456403.47 & +160.37 & $\ldots$ & $16.392(012)$ & $17.195(014)$ & $17.573(019)$ & $16.697(030)$ & $16.542(013)$ \\
\hline 2456412.46 & +169.36 & $\ldots$ & $16.524(013)$ & $17.405(020)$ & $\ldots$ & $\cdots$ & $\cdots$ \\
\hline
\end{tabular}

Note. The phase is relative to $t_{B_{\max }}=\mathrm{JD} 2456243.1$. The values in parentheses are $1 \sigma$ measurement uncertainties and they are given in millimag.

\subsection{Optical Photometry}

\subsubsection{CSP}

Optical imaging of SN 2012fr was obtained with the $1 \mathrm{~m}$ Henrietta Swope telescope located at LCO, which was equipped with the same Johnson $(B V)$ and Sloan (ugri) filter set and CCD detector that were used for the CSP-I (Contreras et al. 2010; Stritzinger et al. 2011; Krisciunas et al. 2017). All of the images were reduced in the manner described by Contreras et al. (2010) and Krisciunas et al. (2017), including the subtraction of the host-galaxy reference images that were obtained after the SN had fully disappeared. PSF photometry of the SN was computed with respect to a local sequence of stars that were calibrated to Landolt (1992) and Smith et al. (2002) standard fields, which were observed over the course of more than 60 photometric nights. The photometry of the local sequence in the standard system is given in Table 2.
The definitive $u B g V r i$-band photometry of SN 2012fr in the Swope natural system (Krisciunas et al. 2017) is given in Table 3 and the corresponding light curves are plotted in individual panels contained within Figure 2. The color curves are also plotted in Figure 3, showing the high quality of the data set. Comprising $\sim 120$ epochs, the light curves track the flux evolution from -12 days to +160 days relative to $t_{B_{\max }}$, representing one of the most comprehensive data sets yet obtained of a Type Ia SN.

As discussed in Contreras et al. (2010), photometry in the natural system is the purest form of the data and it provides the most transparent way to combine CSP photometry with data sets from other groups. Nevertheless, as requested by the referee, we provide S-corrections in Table 4 that, when added to the corresponding optical natural photometry magnitudes in Table 3, convert the photometry to the standard systems; i.e., Landolt (1992) for $B$ and $V$, and Smith et al. (2002) for $u, g, r$, 


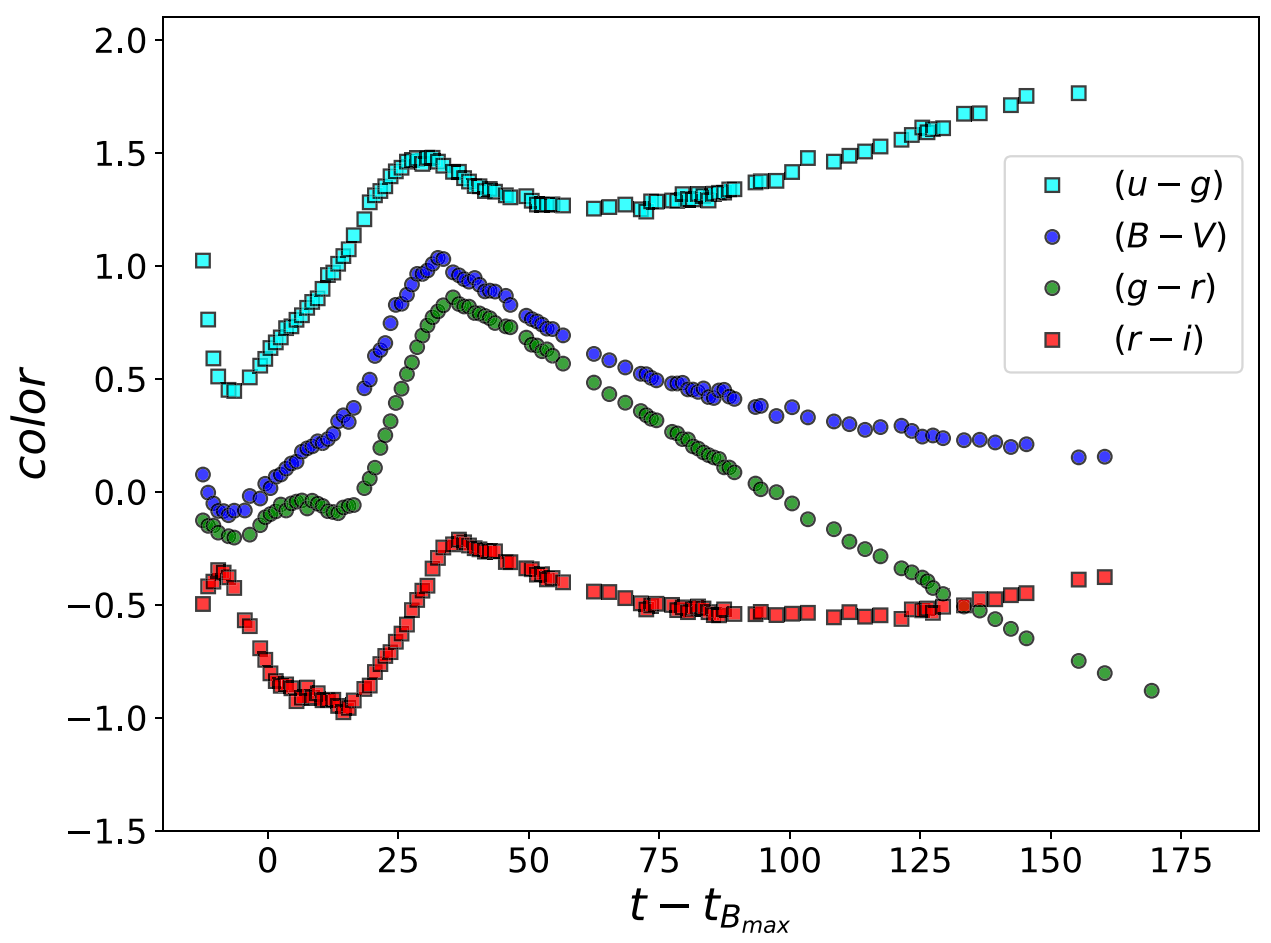

Figure 3. Optical colors of SN 2012fr. The error bars are omitted for the sake of clarity.

and $i$. The S-corrections were calculated using the Hsiao et al. (2007) spectral template and SN 2012fr spectra (Childress et al. 2013) when available (these values are given in parenthesis). The S-corrections are measured after the spectra are slightly altered to match the photometric colors of the corresponding phases.

\subsubsection{TAROT}

SN 2012fr was discovered in images obtained with the robotic telescope that is operated by the TAROT collaboration at the La Silla Observatory. The discovery image was taken on 2012 October 27.05 UT (JD 2456227.55) with an open filter (i.e., without a filter) and follow-up $B V R$ images were obtained beginning two nights later. The telescope optics, filters, and detector are described in detail by Klotz et al. (2008). Throughput functions are presented in Appendix A for the open and $V$ filters. The magnitudes for SN 2012fr and the local standards in the field are derived in the natural systems of these two filters.

\subsubsection{La Silla-QUEST}

In an attempt to better constrain the rise time of the $\mathrm{SN}$, we examined the observing log of the La Silla-QUEST (LSQ) Low Redshift Supernova Survey (Baltay et al. 2013), which went into routine operations approximately one year before the discovery of SN 2012fr. The log indicated that the field of NGC 1365 was observed through a wide-band gr filter every night from 2012 October 23 to 27 UT. Subsequent examination of the images revealed that the $\mathrm{SN}$ was clearly visible on 2012 October 26.19 UT (see Figure 4) but was absent in the images obtained before this date. Unfortunately, the image of the SN was saturated in all of the images that were obtained after 2012 October 26 UT. The details of the telescope and detector system employed for the LSQ survey are given in
Baltay et al. (2007, 2013). Appendix A derives the throughput function of the gr filter, and the natural system magnitudes for the SN and the local standards are presented.

\subsubsection{Slooh}

Klotz \& Conseil (2012) reported that SN 2012fr was confirmed by E. Conseil from images obtained with the $0.5 \mathrm{~m}$ Slooh Space Camera robotic telescope at Mt. Teide, on the island of Tenerife. These observations were made with a set of Astrodon TruBalance $L R G B$ E-Series filters using an FLI PL09000 CCD camera on 2012 October 27.13 UT; i.e., less than two hours after the TAROT discovery image. The Slooh observations are extremely important because they provide information on both the brightness of the $\mathrm{SN}$ and the color shortly after discovery. The throughput functions of the $L R G B$ filters are estimated in Appendix A, and the natural system magnitudes are derived for the SN and the local standards in the field of SN $2012 \mathrm{fr}$.

\subsubsection{BOSS}

Confirming images of SN 2012fr were taken by Stu Parker at Parkdale Observatory in New Zealand, starting 1.3 days after discovery by the TAROT collaboration (Parker 2012). Parker, who is a member of the Backyard Observatory Supernova Search (BOSS), used a $0.36 \mathrm{~m}$ Celestron telescope with an SBIG ST-10XME CCD camera and no filter for these observations. The throughput function of this combination is calculated in Appendix A, which we henceforth refer to as the BOSS open system, and the natural system magnitudes for the $\mathrm{SN}$ and the local standards are derived.

\subsection{NIR Photometry}

The majority of our NIR imaging was obtained on the LCO du Pont $2.5 \mathrm{~m}$ telescope with RetroCam, which is a $Y J H$-band 
Table 4

S-corrections to Standard Photometric Systems

\begin{tabular}{|c|c|c|c|c|c|c|}
\hline Filter & $B$ & $V$ & $u^{\prime}$ & $g^{\prime}$ & $r^{\prime}$ & $i^{\prime}$ \\
\hline 2456230.70 & -0.040 & -0.014 & -0.014 & -0.004 & -0.000 & -0.020 \\
\hline 2456231.70 & -0.036 & -0.015 & 0.002 & -0.004 & -0.000 & -0.016 \\
\hline 2456232.70 & -0.031 & -0.022 & 0.015 & -0.004 & -0.000 & -0.011 \\
\hline 2456233.62 & -0.031 & -0.020 & 0.015 & -0.005 & -0.000 & -0.012 \\
\hline 2456234.66 & -0.024 & -0.014 & 0.030 & -0.006 & -0.000 & -0.018 \\
\hline 2456235.60 & -0.026 & -0.012 & 0.023 & -0.006 & -0.000 & -0.017 \\
\hline 2456236.69 & -0.025 & -0.013 & 0.011 & -0.006 & -0.001 & -0.017 \\
\hline 2456238.72 & -0.020 & $-0.014(-0.008)$ & -0.005 & $-0.005(-0.005)$ & $-0.000(-0.002)$ & $-0.017(-0.013)$ \\
\hline 2456239.67 & -0.025 & $-0.015(-0.004)$ & 0.007 & $-0.005(-0.005)$ & $-0.000(-0.002)$ & $-0.015(-0.013)$ \\
\hline 2456241.67 & -0.026 & -0.016 & 0.039 & -0.005 & 0.000 & -0.016 \\
\hline 2456242.64 & -0.025 & $-0.016(-0.016)$ & 0.041 & $-0.006(-0.005)$ & $0.000(-0.002)$ & $-0.018(-0.022)$ \\
\hline 2456243.63 & -0.023 & -0.017 & 0.051 & -0.006 & 0.000 & -0.020 \\
\hline 2456244.65 & -0.022 & $-0.018(-0.020)$ & 0.053 & $-0.006(-0.006)$ & $0.000(-0.002)$ & $-0.022(-0.025)$ \\
\hline 2456245.61 & -0.020 & $-0.019(-0.011)$ & 0.058 & $-0.006(-0.006)$ & $0.000(-0.002)$ & $-0.024(-0.024)$ \\
\hline 2456246.63 & -0.019 & $-0.020(-0.024)$ & 0.066 & $-0.006(-0.006)$ & $0.000(-0.002)$ & $-0.026(-0.032)$ \\
\hline 2456247.65 & -0.018 & $-0.021(-0.014)$ & 0.078 & $-0.006(-0.007)$ & $0.001(-0.002)$ & $-0.026(-0.031)$ \\
\hline 2456248.62 & -0.016 & $-0.022(-0.015)$ & 0.089 & $-0.006(-0.007)$ & $0.001(-0.002)$ & $-0.028(-0.032)$ \\
\hline 2456249.66 & -0.014 & $-0.022(-0.015)$ & 0.100 & $-0.006(-0.008)$ & $0.001(-0.002)$ & $-0.029(-0.033)$ \\
\hline 2456250.67 & -0.012 & $-0.023(-0.028)$ & 0.112 & $-0.006(-0.007)$ & $0.000(-0.002)$ & $-0.030(-0.038)$ \\
\hline 2456251.63 & -0.011 & $-0.022(-0.029)$ & 0.114 & $-0.006(-0.007)$ & $0.000(-0.002)$ & $-0.030(-0.038)$ \\
\hline 2456252.68 & -0.010 & $-0.021(-0.028)$ & 0.128 & $-0.005(-0.008)$ & $0.000(-0.002)$ & $-0.029(-0.038)$ \\
\hline 2456253.61 & -0.008 & -0.021 & 0.137 & -0.005 & 0.000 & -0.028 \\
\hline 2456254.64 & -0.005 & -0.020 & 0.135 & -0.004 & 0.000 & -0.025 \\
\hline 2456255.67 & -0.005 & -0.020 & 0.136 & -0.002 & 0.000 & -0.022 \\
\hline 2456256.60 & -0.008 & -0.021 & 0.143 & 0.000 & 0.001 & -0.019 \\
\hline 2456257.60 & -0.008 & -0.021 & 0.149 & 0.002 & 0.001 & -0.016 \\
\hline 2456258.62 & -0.003 & -0.022 & 0.153 & 0.002 & 0.000 & -0.014 \\
\hline 2456259.59 & -0.001 & -0.023 & 0.157 & 0.002 & 0.000 & -0.013 \\
\hline 2456261.62 & $0.011(0.006)$ & $-0.025(-0.036)$ & 0.165 & $0.004(-0.002)$ & $0.001(-0.002)$ & $-0.012(-0.034)$ \\
\hline 2456262.65 & 0.013 & -0.027 & 0.161 & 0.006 & 0.002 & -0.013 \\
\hline 2456263.65 & 0.014 & -0.029 & 0.157 & 0.008 & 0.002 & -0.013 \\
\hline 2456264.69 & 0.015 & -0.034 & 0.159 & 0.009 & 0.002 & -0.011 \\
\hline 2456265.63 & 0.014 & -0.039 & 0.162 & 0.012 & 0.003 & -0.008 \\
\hline 2456266.64 & 0.013 & -0.044 & 0.163 & 0.014 & 0.003 & -0.005 \\
\hline 2456267.65 & 0.010 & -0.048 & 0.159 & 0.017 & 0.003 & -0.000 \\
\hline 2456268.74 & 0.007 & -0.052 & 0.154 & 0.020 & 0.003 & 0.004 \\
\hline 2456269.78 & 0.003 & -0.055 & 0.148 & 0.023 & 0.003 & 0.008 \\
\hline 2456270.74 & 0.002 & -0.059 & 0.146 & 0.024 & 0.003 & 0.010 \\
\hline 2456271.74 & 0.005 & -0.061 & 0.144 & 0.025 & 0.003 & 0.012 \\
\hline 2456272.74 & 0.010 & -0.061 & 0.143 & 0.024 & 0.004 & 0.015 \\
\hline 2456273.71 & 0.015 & -0.060 & 0.142 & 0.022 & 0.004 & 0.017 \\
\hline 2456274.69 & 0.018 & -0.059 & 0.138 & 0.021 & 0.004 & 0.019 \\
\hline 2456275.72 & 0.017 & -0.060 & 0.129 & 0.022 & 0.004 & 0.021 \\
\hline 2456276.75 & 0.017 & -0.060 & 0.120 & 0.023 & 0.004 & 0.022 \\
\hline 2456278.61 & $0.016(0.019)$ & $-0.060(-0.068)$ & 0.109 & $0.024(0.021)$ & $0.004(0.002)$ & $0.024(0.002)$ \\
\hline 2456279.74 & 0.015 & -0.058 & 0.100 & 0.024 & 0.004 & 0.025 \\
\hline 2456280.73 & 0.015 & -0.056 & 0.103 & 0.023 & 0.004 & 0.025 \\
\hline 2456281.67 & 0.016 & -0.054 & 0.107 & 0.022 & 0.004 & 0.025 \\
\hline 2456282.76 & 0.017 & -0.050 & 0.112 & 0.020 & 0.003 & 0.025 \\
\hline 2456283.68 & 0.018 & -0.049 & 0.117 & 0.018 & 0.003 & 0.025 \\
\hline 2456284.72 & 0.016 & -0.049 & 0.121 & 0.018 & 0.003 & 0.026 \\
\hline 2456285.65 & 0.013 & -0.050 & 0.125 & 0.018 & 0.003 & 0.028 \\
\hline 2456286.65 & 0.010 & -0.052 & 0.128 & 0.019 & 0.003 & 0.029 \\
\hline 2456288.73 & 0.006 & -0.055 & 0.131 & 0.021 & 0.003 & 0.030 \\
\hline 2456289.60 & 0.006 & -0.055 & 0.134 & 0.021 & 0.003 & 0.031 \\
\hline 2456292.65 & 0.009 & -0.052 & 0.140 & 0.020 & 0.003 & 0.033 \\
\hline 2456293.69 & 0.010 & -0.051 & 0.142 & 0.019 & 0.002 & 0.034 \\
\hline 2456294.68 & 0.012 & -0.050 & 0.144 & 0.018 & 0.002 & 0.035 \\
\hline 2456295.66 & 0.012 & -0.047 & 0.144 & 0.017 & 0.002 & 0.036 \\
\hline 2456296.60 & 0.013 & $-0.048(-0.075)$ & 0.144 & 0.018 & $0.002(-0.002)$ & $0.036(0.015)$ \\
\hline 2456297.67 & 0.014 & -0.047 & 0.144 & 0.018 & 0.002 & 0.037 \\
\hline 2456299.69 & 0.016 & -0.046 & 0.144 & 0.017 & 0.002 & 0.039 \\
\hline 2456305.60 & 0.012 & -0.040 & 0.145 & 0.013 & 0.001 & 0.039 \\
\hline 2456308.55 & 0.010 & -0.043 & 0.148 & 0.014 & 0.001 & 0.036 \\
\hline
\end{tabular}


Table 4

(Continued)

\begin{tabular}{lrrrrrr}
\hline \hline Filter & $B$ & $V$ & $u^{\prime}$ & $g^{\prime}$ & $r^{\prime}$ \\
\hline 2456311.61 & -0.003 & -0.068 & 0.151 & 0.017 & 0.003 & 0.040 \\
2456314.59 & 0.010 & -0.039 & 0.153 & 0.014 & -0.000 & 0.000 \\
2456315.63 & 0.011 & -0.043 & 0.151 & 0.014 & -0.000 & 0.038 \\
2456316.56 & 0.012 & -0.043 & 0.150 & 0.014 & -0.000 & 0.037 \\
2456317.58 & 0.013 & -0.042 & 0.148 & 0.013 & -0.001 & 0.037 \\
2456320.57 & 0.017 & -0.039 & 0.144 & 0.011 & -0.001 & 0.035 \\
2456321.56 & 0.018 & -0.038 & 0.142 & 0.011 & -0.001 & 0.034 \\
2456322.59 & 0.018 & -0.038 & 0.142 & 0.011 & -0.001 \\
2456323.62 & 0.019 & -0.037 & 0.140 & 0.010 & 0.034 & 0.033 \\
\hline
\end{tabular}

Note. S-corrections, as given by $M_{\text {std }}=M_{\text {nat }}+S_{M}$, are calculated for all phases using the Hsiao et al. (2007) spectral template. For those phases where a spectrum of SN 2012fr exists (Childress et al. 2013), the S-correction calculated from that spectrum is given in parentheses. For $B$ and $V$, the filter functions and zero points are taken from Stritzinger et al. (2005). For $u, g$, r, and $i$, the BD $+17^{\circ} 4708$ spectrum that is given by Bohlin et al. (2014) is used, along with Fukugita et al.'s (1996) filter functions and Smith et al.'s (2002) standard magnitudes to obtain photometric zero points.

imager employing a Hawaii-1 HgCdTe $1024 \times 1024$ pixel array. The field of view of RetroCam at the du Pont telescope is $3.5 \times 3 ! 5$, with a pixel scale of 0 !' 20 . Additional NIR imaging of SN 2012fr was obtained in $J 1, J$, and $H$ filters using the FourStar camera attached to the $6.5 \mathrm{~m}$ Magellan Baade telescope (Persson et al. 2013). FourStar consists of a mosaic of four Hawaii-2RG HgCdTe detectors and each chip yields a field of view of $5^{\prime} \times 5^{\prime}$. The RetroCam and FourStar bandpasses are illustrated in Figure 5. It can be seen from this figure that the FourStar $J 1$ filter covers $\sim 75 \%$ of the wavelength range of the RetroCam $Y$ filter.

The NIR images were reduced in the standard manner following the steps described by Contreras et al. (2010). In short, images were dark subtracted, flat fielded (and sky + fringing subtracted in the case of RetroCam images), and each dithered frame was aligned and combined. Host-galaxy reference images were subtracted from each combined image on a $15^{\prime \prime}$ radius circle around the $\mathrm{SN}$.

The NIR photometry of SN 2012fr was computed differentially with respect to a local sequence of stars, which were defined using RetroCam observations. The local sequence was calibrated in the Persson et al. (1998) $J H$ photometric system using standard star fields that were observed during 10 photometric nights. For the $Y$-band, the local sequence stars were calibrated using the magnitudes for a subset of Persson et al. standards, as published by Krisciunas et al. (2017). The final NIR $Y$, J, and $H$ magnitudes for the local sequence stars in these standard systems are listed in Table 2.

To compute magnitudes for the local sequence stars in the natural system of the three FourStar filters (hereafter, referred to as $J 1_{\mathrm{FS}}, J_{\mathrm{FS}}$, and $H_{\mathrm{FS}}$ ), the following set of transformation relations were obtained via synthetic photometry of Castelli \& Kurucz's (2003) model atmospheres:

$$
\begin{gathered}
Y_{\mathrm{std}}=J 1_{\mathrm{FS}}+0.1064(J-H)_{\mathrm{std}}+0.0052, \\
J_{\mathrm{std}}=J_{\mathrm{FS}}+0.0008(J-H)_{\mathrm{std}}+0.0047, \\
H_{\mathrm{std}}=H_{\mathrm{FS}}-0.0395(J-H)_{\mathrm{std}}+0.0062 .
\end{gathered}
$$

In these equations, $J_{\text {std }}$ and $H_{\text {std }}$ are the magnitudes in Persson et al.'s standard system; $Y_{\text {std }}$ is the magnitude in the RetroCam $Y$-band standard system, as defined by Krisciunas et al. (2017); $J_{\mathrm{FS}}, J 1_{\mathrm{FS}}$, and $H_{\mathrm{FS}}$ are the magnitudes in natural system of
FourStar; and $(J-H)_{\text {std }}$ is the color index of the star in Persson et al.'s system. The Persson et al. and FourStar filter functions that are used to derive the synthetic magntiudes are made available on the CSP website. ${ }^{26}$

Making use of the local sequence, we now proceeded to compute the definitive NIR photometry of SN 2012fr in the natural system of the RetroCam $Y J H$ filters (hereafter, referred to as $Y_{\mathrm{RC}}, J_{\mathrm{RC}}$, and $H_{\mathrm{RC}}$, as in Krisciunas et al. 2017), which is listed in Table 5.

This photometry is plotted in individual panels contained within Figure 2. Consisting of more than 40 epochs, the light curves follow the flux evolution from -11 to +140 days with respect to $t_{B_{\max }}$. The FourStar $J 1_{\mathrm{FS}}, J_{\mathrm{FS}}$, and $H_{\mathrm{FS}}$ photometry was S-corrected (Suntzeff 2000; Stritzinger et al. 2002) to match the RetroCam $Y_{\mathrm{RC}}, J_{\mathrm{RC}}$, and $H_{\mathrm{RC}}$ system using the following spectrophotometric prescription:

1. Spectral templates (Hsiao et al. 2007) matching the phases of each FourStar photometry epoch were employed. The template spectrum for each FourStar epoch was color matched separately to the photometry in the $J 1_{\mathrm{FS}}, J_{\mathrm{FS}}$, and $H_{\mathrm{FS}}$ bands, respectively.

2. For the $J 1_{\mathrm{FS}}$ band, the color-matching function was a second order polynomial calculated using CSP $i$ and FourStar $J 1_{\mathrm{FS}}$ and $J_{\mathrm{FS}}$ photometry. Likewise, for the $J_{\mathrm{FS}}$ band, a second order polynomial was derived from the $J 1_{\mathrm{FS}}, J_{\mathrm{FS}}$, and $H_{\mathrm{FS}}$ photometry. For the $H_{\mathrm{FS}}$ band, a linear polynomial was employed to color match the template to the $J_{\mathrm{FS}}$ and $H_{\mathrm{FS}}$ magnitudes.

3. The S-correction was then derived as the difference between the synthetic RetroCam and FourStar magnitudes derived from the mangled spectrum. Specifically: $S_{J 1_{\mathrm{FS}}}=Y_{\mathrm{RC}}-J 1_{\mathrm{FS}} ; \quad S_{J_{\mathrm{FS}}}=J_{\mathrm{RC}}-J_{\mathrm{FS}} ; \quad$ and $\quad S_{H_{\mathrm{FS}}}=$ $H_{\mathrm{RC}}-H_{\mathrm{FS}}$.

The resulting $\mathrm{S}$-corrections are listed in Table 6. The excellent consistency between the $S$-corrected FourStar photometry and the RetroCam observations is illustrated in Figure 6. This is confirmed by looking at nights where nearly simultaneous measurements were made with both instruments. For the $Y$-band at phases of -8.3 and -7.3 days, we find differences between the S-corrected FourStar and RetroCam photometry of $-0.022 \pm 0.030$ and $-0.006 \pm 0.031 \mathrm{mag}$,

\footnotetext{
${ }^{26}$ http://csp.obs.carnegiescience.edu/
} 

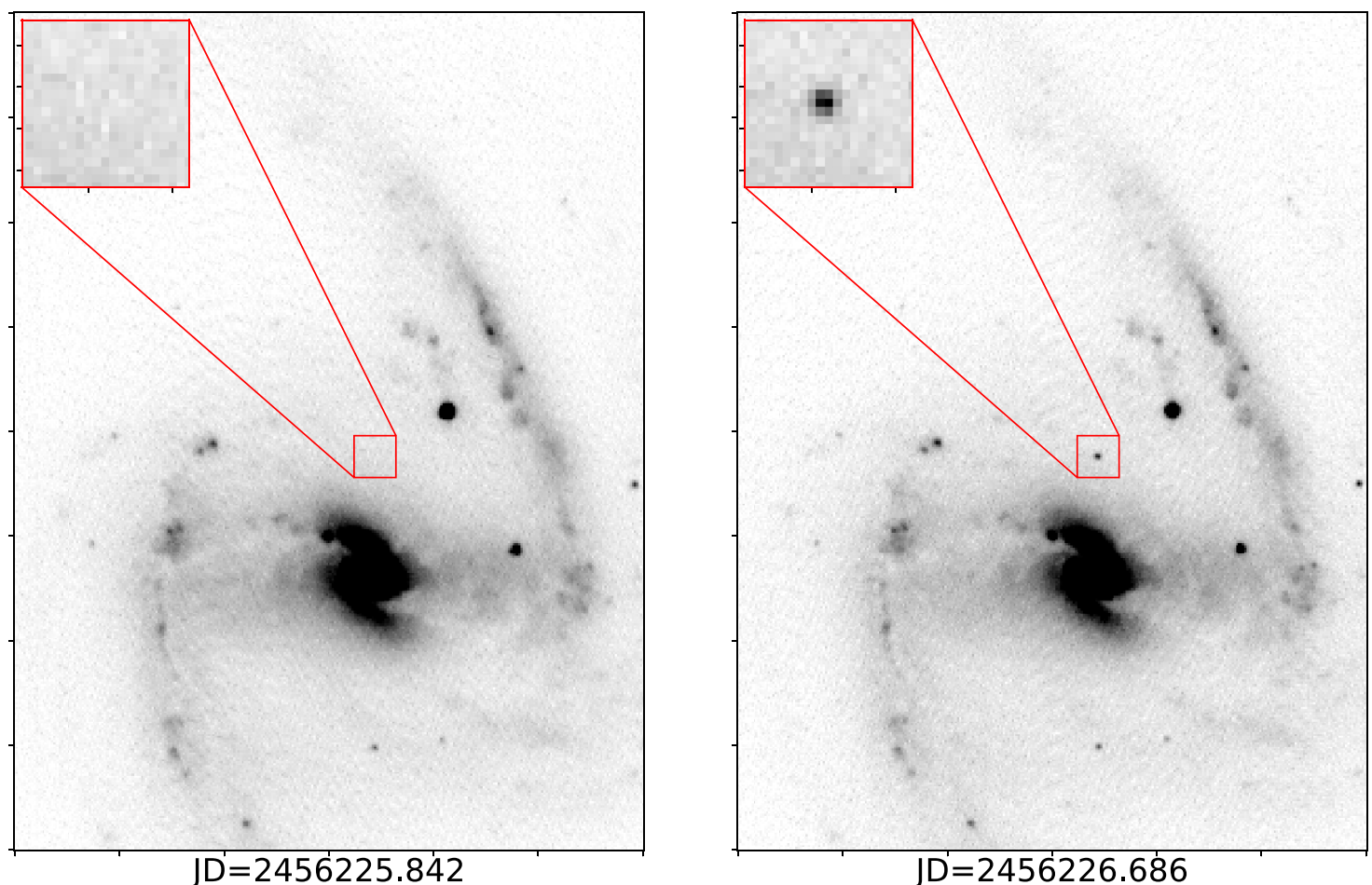

Figure 4. LSQ Survey images of NGC 1365 taken on 2012 October 25.34 UT and 2012 October 26.19 UT. The position of SN $2012 \mathrm{fr}$ is magnified in the upper lefthand corner of both images.

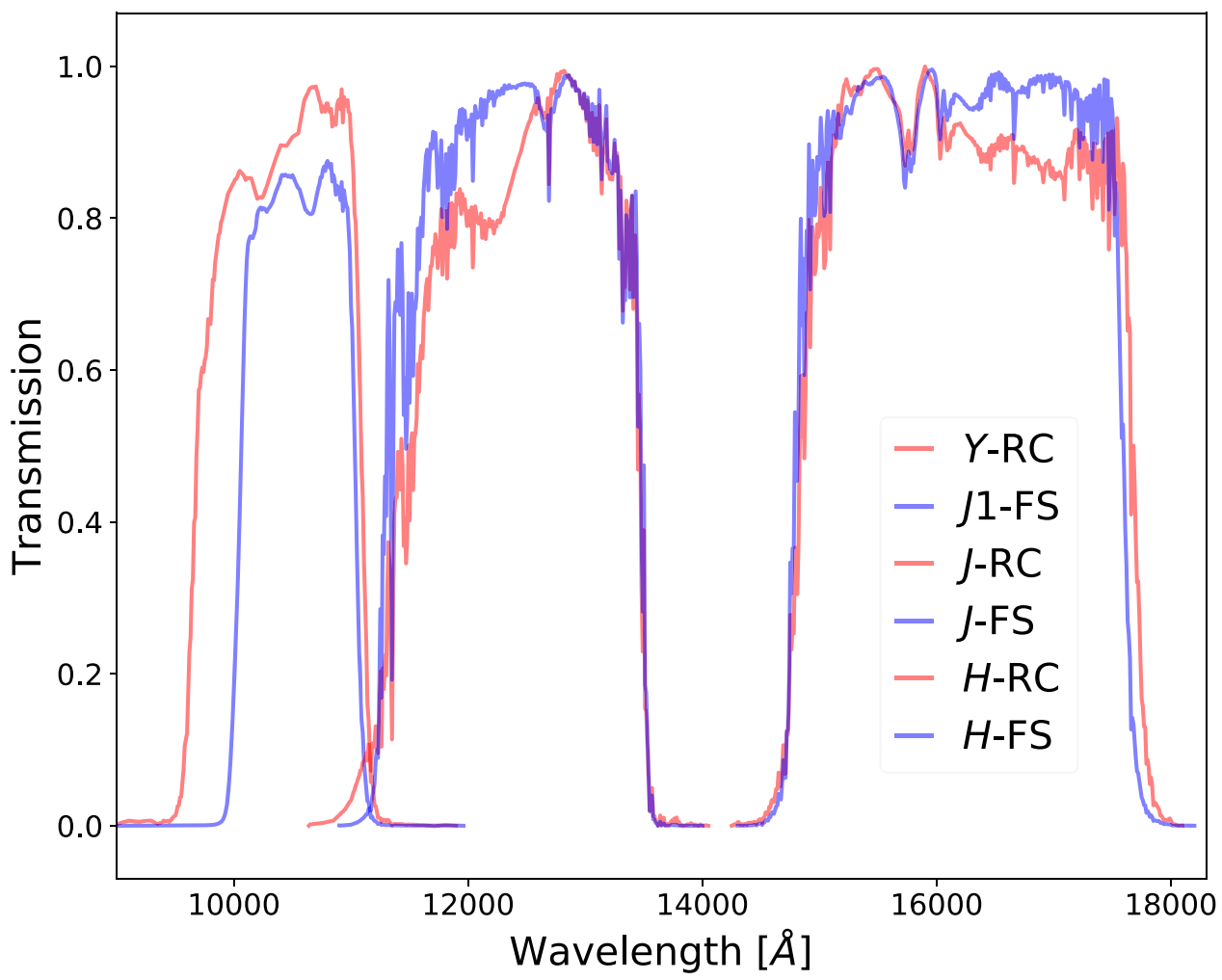

Figure 5. Comparison between the transmission functions of the Swope + RetroCam $Y_{\mathrm{RC}}, J_{\mathrm{RC}}$, and $H_{\mathrm{RC}}$ filters and the Magellan Baade + FourStar $J 1_{\mathrm{FS}}, J_{\mathrm{FS}}$, and $H_{\mathrm{FS}}$ filters.

respectively. For the $J$ band at phases of $-8.3,-7.3$, and +53.5 days, the differences are $-0.016 \pm 0.028,-0.017 \pm$ 0.028 , and $+0.035 \pm 0.041 \mathrm{mag}$, respectively, while for the $H$ band at the same phases, the differences are $-0.061 \pm 0.068$, $-0.020 \pm 0.060$, and $0.000 \pm 0.001 \mathrm{mag}$.

\section{Analysis}

\subsection{Light-Curve Parameters}

The densely sampled light curves of SN 2012fr allow us to accurately measure the apparent magnitude at maximum and 
Table 5

Near-infrared Photometry of SN 2012fr in the Natural System of du Pont

\begin{tabular}{|c|c|c|c|c|c|}
\hline JD (days) & Phase (days) & $Y(\mathrm{mag})$ & $J$ (mag) & $H$ (mag) & Telescope \\
\hline 2456231.84 & -11.26 & $13.675(027)$ & $13.607(033)$ & $13.712(031)$ & BAA \\
\hline 2456232.87 & -10.23 & $13.390(022)$ & $13.302(020)$ & $13.507(029)$ & BAA \\
\hline 2456233.86 & -9.24 & $13.177(022)$ & $13.069(020)$ & $13.269(029)$ & BAA \\
\hline 2456234.71 & -8.39 & $13.061(025)$ & $12.953(020)$ & $13.148(061)$ & DUP \\
\hline 2456234.85 & -8.25 & $13.028(022)$ & $12.938(020)$ & 13.207(029) & BAA \\
\hline 2456235.73 & -7.37 & $12.979(022)$ & $12.848(020)$ & $13.094(061)$ & DUP \\
\hline 2456235.84 & -7.26 & $12.974(022)$ & $12.834(020)$ & $13.072(031)$ & BAA \\
\hline 2456236.67 & -6.43 & $12.929(025)$ & $12.799(020)$ & $12.974(062)$ & DUP \\
\hline 2456237.60 & -5.50 & $12.894(025)$ & $12.745(020)$ & $12.990(061)$ & DUP \\
\hline 2456241.86 & -1.24 & $12.988(026)$ & $12.776(025)$ & $13.040(027)$ & BAA \\
\hline 2456244.87 & +1.77 & $13.224(022)$ & $12.901(020)$ & $13.134(027)$ & BAA \\
\hline 2456246.86 & +3.76 & $13.482(022)$ & $13.048(020)$ & $13.185(029)$ & BAA \\
\hline 2456248.87 & +5.77 & $13.624(022)$ & $13.176(020)$ & $13.235(028)$ & BAA \\
\hline 2456249.87 & +6.77 & $13.648(022)$ & $13.286(020)$ & $13.316(030)$ & BAA \\
\hline 2456251.86 & +8.76 & $13.780(022)$ & $13.545(020)$ & $13.398(040)$ & BAA \\
\hline 2456252.84 & +9.74 & $13.885(027)$ & $13.639(020)$ & $13.389(027)$ & BAA \\
\hline 2456253.69 & +10.59 & $13.861(022)$ & $13.807(020)$ & $13.383(027)$ & DUP \\
\hline 2456254.68 & +11.58 & $13.899(022)$ & $13.984(020)$ & $13.440(027)$ & DUP \\
\hline 2456256.84 & +13.74 & $13.949(022)$ & $14.335(020)$ & $13.450(028)$ & BAA \\
\hline 2456258.59 & +15.49 & $13.911(026)$ & $14.521(020)$ & $13.444(062)$ & DUP \\
\hline 2456259.60 & +16.50 & $13.875(025)$ & $14.530(020)$ & $13.398(061)$ & DUP \\
\hline 2456264.59 & +21.49 & $13.525(022)$ & $14.459(020)$ & $13.287(027)$ & DUP \\
\hline 2456265.61 & +22.51 & $13.459(026)$ & $14.432(025)$ & $13.254(030)$ & DUP \\
\hline 2456266.61 & +23.51 & $13.381(026)$ & $14.393(025)$ & $13.218(030)$ & DUP \\
\hline 2456281.57 & +38.47 & $12.660(022)$ & $13.898(020)$ & 13.291(027) & BAA \\
\hline 2456284.60 & +41.50 & $12.911(022)$ & $14.163(020)$ & $13.502(027)$ & DUP \\
\hline 2456287.75 & +44.65 & $13.094(022)$ & $14.438(025)$ & $13.698(028)$ & DUP \\
\hline 2456293.61 & +50.51 & $13.437(026)$ & $14.871(025)$ & $13.909(030)$ & DUP \\
\hline 2456295.56 & +52.46 & $13.522(022)$ & $15.017(020)$ & $13.972(027)$ & DUP \\
\hline 2456296.54 & +53.44 & $\cdots$ & $15.051(033)$ & $14.039(027)$ & BAA \\
\hline 2456296.62 & +53.52 & $13.595(026)$ & $15.092(025)$ & $14.041(030)$ & DUP \\
\hline 2456297.66 & +54.56 & $13.655(026)$ & $15.148(025)$ & $14.085(030)$ & DUP \\
\hline 2456298.53 & +55.43 & $13.661(022)$ & $15.131(020)$ & $14.128(027)$ & BAA \\
\hline 2456304.54 & +61.44 & $13.967(022)$ & $15.555(020)$ & $14.350(029)$ & BAA \\
\hline 2456318.59 & +75.49 & $14.767(022)$ & $16.388(021)$ & $14.955(035)$ & DUP \\
\hline 2456326.57 & +83.47 & $15.170(022)$ & $16.859(021)$ & $15.280(029)$ & DUP \\
\hline 2456327.56 & +84.46 & $15.222(022)$ & $16.900(021)$ & 15.293(061) & DUP \\
\hline 2456342.60 & +99.50 & $15.861(025)$ & $17.587(025)$ & $15.926(063)$ & DUP \\
\hline 2456343.59 & +100.49 & $15.905(034)$ & $17.565(028)$ & $16.028(054)$ & DUP \\
\hline 2456345.57 & +102.47 & $16.003(026)$ & $17.693(039)$ & $16.092(033)$ & DUP \\
\hline 2456346.55 & +103.45 & $16.023(027)$ & $17.709(033)$ & $16.122(034)$ & DUP \\
\hline 2456349.58 & +106.48 & $16.138(026)$ & $17.741(031)$ & $16.134(064)$ & DUP \\
\hline 2456352.58 & +109.48 & $16.215(025)$ & $17.871(022)$ & $16.223(062)$ & DUP \\
\hline 2456378.51 & +135.41 & $16.911(022)$ & $18.591(044)$ & $16.897(045)$ & DUP \\
\hline 2456382.53 & +139.43 & $17.003(023)$ & $18.723(036)$ & $16.984(044)$ & DUP \\
\hline
\end{tabular}

Note. The values in parentheses are $1 \sigma$ measurement uncertainties and they are given in millimag. The phase is relative to $t_{B_{\max }}=\mathrm{JD} 2456243.1$.

the decline-rate parameter, $\Delta \mathrm{m}_{15}(X),{ }^{27}$ in each filter, $X$, using a smooth Gaussian process's fitting curve. The values are summarized in Table 7. K-corrections were ignored as the small redshift $\left(z_{\text {helio }}=0.005457\right.$ according to $\mathrm{NED}^{28}$ ) of the hostgalaxy, NGC 1365, has very little effect on these quantities. In addition, absolute magnitudes are provided as computed using the adopted average Cepheid distance discussed in Section 3.3 and assuming a host-galaxy dust reddening of $E(B-V)_{\text {host }}=$

\footnotetext{
${ }^{27}$ The decline-rate parameters, $\Delta m_{15}(X)$, are here defined as the magnitude difference of the light curve in filter $X$ from peak to 15 days later. Historically, the value of $\Delta m_{15}(B)$ was shown by Phillips (1993) to correlate with the absolute peak magnitude in such a way that more luminous objects show slower light curve decline rates.

${ }^{28}$ NED is the NASA/IPAC Extragalactic Database.
}

$0.03 \pm 0.03$ mag (see Section 3.2). The fit to the $B$-band light curve indicates that $t_{B_{\max }}$ occurred on JD 2456243.1 \pm 0.3 and $\Delta m_{15}(B)=0.82 \pm 0.03 \mathrm{mag}$. This rather slow decline-rate implies that SN $2012 \mathrm{fr}$ should be moderately over-luminous compared to a standard SN Ia with $\Delta m_{15}(B)=1.1 \mathrm{mag}$ and an absolute $B$-band magnitude $M_{B}=-19.1 \mathrm{mag}$ (Folatelli et al. 2010). Indeed, as shown in Table 7, SN 2012fr was $\sim 0.3 \mathrm{mag}$ more luminous than this value.

As expected for a normal SN Ia, the NIR light curves reached primary maxima $\sim 4-5$ days prior to $t_{B_{\max }}$. The absolute magnitudes in $Y, J$, and $H$ were calculated using the Cepheid distance to NGC 1365 and they are given in Table 7. These values are also fully consistent with the average values for slow-to-mid-decliners given by Kattner et al. (2012) in Table 7 . 
Table 6

Baade FourStar S-corrections for SN 2012fr

\begin{tabular}{lccr}
\hline \hline Phase (days) & $S_{J_{\mathrm{FS}}}(\mathrm{mag})$ & $S_{J_{\mathrm{FS}}}(\mathrm{mag})$ & $S_{H_{\mathrm{FS}}}(\mathrm{mag})$ \\
\hline-11 & 0.044 & 0.002 & 0.001 \\
-10 & 0.032 & 0.001 & 0.002 \\
-09 & 0.024 & -0.001 & 0.002 \\
-08 & 0.011 & -0.001 & 0.002 \\
-07 & -0.001 & -0.003 & 0.002 \\
-01 & -0.036 & -0.003 & 0.000 \\
+02 & -0.012 & -0.007 & 0.000 \\
+04 & -0.021 & -0.010 & 0.001 \\
+06 & -0.016 & -0.009 & 0.003 \\
+07 & -0.017 & -0.007 & 0.005 \\
+09 & -0.024 & -0.005 & 0.006 \\
+10 & -0.017 & -0.007 & 0.006 \\
+14 & -0.027 & -0.006 & -0.013 \\
+38 & 0.010 & -0.004 & 0.001 \\
+53 & $\ldots$ & -0.006 & -0.002 \\
+55 & 0.063 & -0.008 & -0.001 \\
+61 & 0.048 & -0.013 & -0.004 \\
\hline
\end{tabular}

Note. The S-correction values were applied as: $X_{\text {Retrocam }}=X_{\text {Fourstar }}+\mathrm{S}$-corr. The phase was computed relative to $t_{B_{\max }}=\mathrm{JD} 2456243.1$ and rounded to match the phases of Hsiao et al. (2007) spectral templates.

\subsection{Host-Galaxy Reddening}

To determine if SN 2012fr suffered any reddening due to dust external to the Milky Way, we make use of three methods. In the first method, the $(B-V)$ color curve of SN 2012fr is compared to the Lira relation (Lira 1995; Phillips et al. 1999). The Lira relation relies on the fact that the $(B-V)$ color of normal unreddened SNe Ia follows a linear evolution with minimal scatter between 30 and 90 days past maximum (see Hoeflich et al. 2017 for a description of the physics underlying the Lira relation). Therefore, once corrected for galactic reddening, comparing the $(B-V)$ color curve of any given SN Ia to the Lira relation provides an indication of the amount of dust reddening external to the Milky Way. Burns et al. (2014) provide the following fit to the Lira relation for a $\mathrm{SN}$ with $\Delta m_{15}(B)=0.82 \mathrm{mag}$ based on the CSP-I data releases 1 and 2 (Contreras et al. 2010; Stritzinger et al. 2011):

$$
\begin{aligned}
(B-V)_{\text {Lira }}= & 0.78( \pm 0.04)-0.0094( \pm 0.0005) \\
& \times\left[t-t_{B_{\max }}-45\right] .
\end{aligned}
$$

This calibration differs somewhat from an analysis done previously by Folatelli et al. (2010), who used a smaller sample of $\mathrm{SNe}$ Ia that was presumed to be unreddened. Meanwhile, Burns et al. (2014) used all of the SNe in the CSP-I sample with good photometric coverage extending beyond 40 days past $t_{B_{\max }}$. The late-time slopes of the $(B-V)$ light curves were measured, in addition to the value of $(B-V)$, at 45 days after $t_{B_{\max }}$ for each object separately. The median slope is used for the Lira law and the median absolute deviation is taken as its uncertainty. The observed distribution of $(B-V)$ colors at day +45 is modeled as the convolution of an intrinsic Gaussian distribution and exponential tail, which is similar to Jha et al. (2007) . The maximum and standard deviation of the resulting Gaussian distribution are taken as the Lira intercept and uncertainty, respectively.

Figure 7 illustrates a plot of the galactic reddening-corrected $(B-V)$ color evolution of SN 2012fr from 25 to 95 days past $t_{B_{\max }}$. The Lira relation from Burns et al. (2014) is over-plotted as a solid line, as defined in Equation (4). Also shown for reference is the relation given by Folatelli et al. (2010). The comparison between the color evolution and the Lira relation from Burns et al. implies that SN 2012fr suffered minimal hostgalaxy reddening, $E(B-V)_{\text {host }}=0.03 \pm 0.04$ mag. However, the excellent precision and sampling of the observations clearly reveal that the $(B-V)$ temporal evolution of SN 2012fr was not linear at these epochs. From 35 to 60 days past $t_{B_{\max }}$, the slope is approximately -0.014 mag day $^{-1}$, whereas from 60-95 days it is approximately -0.008 mag day $^{-1}$. As shown by Burns et al. (2014) in their Figure 12, these values cover the range of slopes displayed by normal SNe Ia. Nevertheless, it is unusual to observe such a large change of slope in any single event.

The second method that we adopted to estimate the hostgalaxy $E(B-V)_{\text {host }}$ color excess relies on well-defined relations between maximum light intrinsic pseudo-colors29 and the decline-rate derived from a large sample of SNe Ia. This method is more often applicable to SNe Ia observations because it makes use of maximum light observations, which are normally more readily available than the post-maximum regime required for an accurate Lira relation analysis. This method is fully detailed in Burns et al. (2014) and we briefly describe it here.

The observed colors $(B-X)$, where $X$ represents each filter except for $B$, are modeled as the sum of an intrinsic color that depends on the decline-rate parameter of the SN, the color excess $E(B-X)_{\mathrm{MW}}$ from the Milky Way dust, and the color excess $E(B-X)_{\text {host }}$ from the host-galaxy ISM. The intrinsic colors are derived from an MCMC analysis of a training sample of SNe Ia from the CSP-I (Burns et al. 2014). The Milky Way component of the reddening is determined by assuming a value $E(B-V)_{\text {MW }}$ from the Schlafly \& Finkbeiner (2011) dust maps, a fixed value for the ratio of total-to-selective absorption $R_{V}^{\mathrm{MW}}=3.1$, and Fitzpatrick's (1999) reddening law. Finally, the host-galaxy component of the reddening is modeled with Fitzpatrick's (1999) reddening law and two free parameters: $E(B-V)_{\text {host }}$ and $R_{V}^{\text {host }}$, which are determined using MCMC methods. The resulting reddening $E(B-V)_{\text {host }}=$ $0.06 \pm 0.02 \mathrm{mag}$ is quite low and, as a result, the posterior of $R_{V}^{\text {host }}=4.2 \pm 1.3$ more closely resembles the population distribution of $R_{V}$ from the training sample rather than the observed colors of SN 2012fr.

The NaI D interstellar absorption lines can be used to provide a third estimate of the host-galaxy reddening of SN 2012fr. For this purpose, we use the Keck HIRES echelle spectrum that was published by Childress et al. (2012). These authors measured weak absorption in the D1 and D2 lines due to gas in the Milky Way at a combined equivalent width (EW) of $118 \pm 19 \mathrm{m \AA}$. Employing the fit to EW(Na I D) versus $A_{V}$ for Galactic stars using Munari \& Zwitter's (1997) relation, as shown in Phillips et al. (2013) in their Figure 9, this value implies $A_{V}=0.05 \pm 0.03 \mathrm{mag}$. This is in excellent agreement with the Schlafly \& Finkbeiner (2011) value given in Section 1. No Na ID absorption at the redshift of NGC 1365 is visible in the echelle spectrum of Childress et al. at a $3 \sigma$ upper limit of $\mathrm{EW}(\mathrm{Na}$ I D $)=82.8 \mathrm{m \AA}$, implying $A_{V} \leqslant 0.04 \pm 0.03 \mathrm{mag}$, or $E(B-V)_{\text {host }}=0.01 \pm 0.01 \mathrm{mag}$ if the interstellar gas has similar properties to the gas in the solar neighborhood.

\footnotetext{
29 A pseudo-color is defined as the difference between peak magnitudes of two passbands. In the case of SNe Ia, the time of peak brightness can vary by up to a few days from passband to passband.
} 

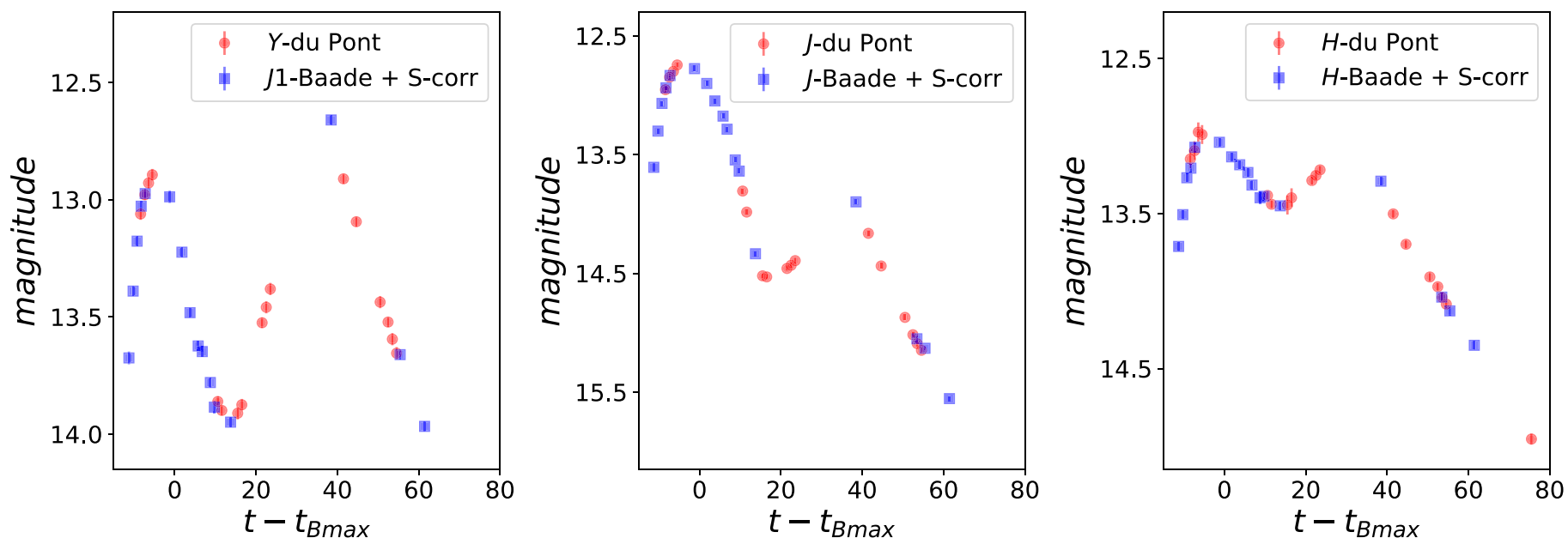

Figure 6. $Y J H$ light curves of SN $2012 \mathrm{fr}$ from -12 to +60 days relative to $t_{B_{\max }}$, acquired with the du Pont (+RetroCam) and the Magellan Baade (+FourStar) telescopes. The photometry obtained between the two facilities matches exceptionally well. In particular, note the excellent agreement between the FourStar S-corrected $J 1 \rightarrow Y$-band and RetroCam $Y$-band photometry.

Table 7

SN 2012fr: Light-curve Parameters and Absolute Magnitudes

\begin{tabular}{|c|c|c|c|c|c|c|}
\hline Band & $\begin{array}{c}t_{\max }(\mathrm{JD}) \\
\text { (days) }\end{array}$ & $\begin{array}{l}\Delta t_{\text {peak }} \\
\text { (days) }\end{array}$ & $\begin{array}{c}m_{\text {Xpeak }} \\
(\mathrm{mag})\end{array}$ & $\underset{(\mathrm{mag})}{m_{\text {Xpeak }}-A_{X}}$ & $\begin{array}{l}\Delta_{m 15} \\
\text { (mag) }\end{array}$ & $\begin{array}{c}M_{X \text { Ceph }} \\
(\mathrm{mag})\end{array}$ \\
\hline$u$ & $2456240.5 \pm 0.3$ & -2.8 & $12.59(02)$ & $12.36(15)$ & $0.87(03)$ & $-18.91(16)$ \\
\hline$B$ & $2456243.1 \pm 0.3$ & +0.0 & $12.04(02)$ & $11.84(13)$ & $0.82(03)$ & $-19.43(14)$ \\
\hline V & $2456245.1 \pm 0.3$ & +2.0 & $11.99(02)$ & $11.84(10)$ & $0.67(03)$ & $-19.43(11)$ \\
\hline$r$ & $2456245.7 \pm 0.3$ & +2.6 & $12.06(02)$ & $11.93(08)$ & $0.79(03)$ & $-19.34(09)$ \\
\hline$i$ & $2456240.1 \pm 0.3$ & -3.0 & $12.84(02)$ & $12.74(07)$ & $0.59(03)$ & $-18.53(09)$ \\
\hline$H$ & $2456238.6 \pm 1.0$ & -4.5 & $12.99(02)$ & $12.96(02)$ & $0.39(03)$ & $-18.31(05)$ \\
\hline$L_{\text {UVOIR }}$ & $2456242.7 \pm 0.3$ & -0.4 & ... & $\ldots$ & $\ldots$ & $\ldots$ \\
\hline
\end{tabular}

$\Delta t_{\text {peak }}=t_{\max }-t_{\mathrm{Bmax}}$

${ }_{A X}$ : Total reddening, i.e., Milky Way plus host-galaxy reddening.

Note. The values in parentheses are $1 \sigma$ measurement uncertainties and they are given in hundredths of a magnitude.

Taken together, these three estimates are consistent with zero or negligible host-galaxy reddening of SN 2012fr. In the rest of this paper, we adopt a value of $E(B-V)_{\text {host }}=$ $0.03 \pm 0.03 \mathrm{mag}$, which is consistent with all three estimates. We also assume $R_{V}=3.1$, but the exact value is not critical since the reddening is low.

\subsection{Distance to NGC 1365 as Derived from SN $2012 f r$}

From the fits to the observed light curves of SN 2012fr using the SNooPy EBV method (Burns et al. 2011), the SN-based distance to NGC 1365 is found to be $\mu_{\circ}=31.25 \pm$ $0.01_{\text {stat }} \pm 0.08_{\text {syst }}$ mag. SNooPy also offers an alternative way to estimate the distance to the host-galaxy using the broadband light curves through the Tripp (1998) method. The functional form of the Tripp method relates the distance modulus of a SN Ia to its decline rate and color via:

$$
\mu_{\circ}=m_{X}^{\max }-M_{X}(0)-b_{X} \cdot\left[\Delta m_{15}-1.1\right]-\beta_{X}^{Y Z} \cdot(Y-Z) .
$$

Here $m_{X}^{\max }$ is the observed K-corrected and galactic-reddening corrected magnitude at maximum, $M_{X}^{0}$ is the peak absolute magnitude of SNe Ia with $\Delta m_{15}=1.1$ and zero dust extinction, $b_{X}$ is the slope of the luminosity versus decline-rate relation, $\beta_{X}^{Y Z}$ is the slope of the luminosity-color relationship, and $(Y-Z)$ is a pseudo-color at maximum. Note that the SNooPy parameter, $\Delta m_{15}$, is the template-derived value of the declinerate parameter, which correlates strongly with the directly measured value, $\Delta m_{15}(B)$, but with some random and systematic deviations (see Figure 6 of Burns et al. 2011).

The Tripp method requires an accurate calibration between the relations of peak absolute magnitude versus $\Delta m_{15}$ and pseudo-color. Here the calibrations presented by Folatelli et al. (2010) in Table 8, lines 2 and 6 are adopted, which are based on 26 well-observed SN Ia light curves published by Contreras et al. (2010) for the $B$-band, and 21 well-observed SN Ia light curves for $J$ band of the same paper. The resulting estimates of distance modulus for NGC 1365 based on the Tripp method are $\mu_{B}=31.14 \pm 0.15 \mathrm{mag}$ and $\mu_{J}=31.34 \pm 0.14 \mathrm{mag}$.

These results are consistent with Freedman et al.'s (2001) Cepheid distance modulus of $\mu=31.27 \pm 0.05 \mathrm{mag}$, which was adopted in this work, indicating that SN 2012fr had a luminosity consistent with a normal SNe Ia with $\Delta m_{15}(B)=$ $0.82 \mathrm{mag}$. 


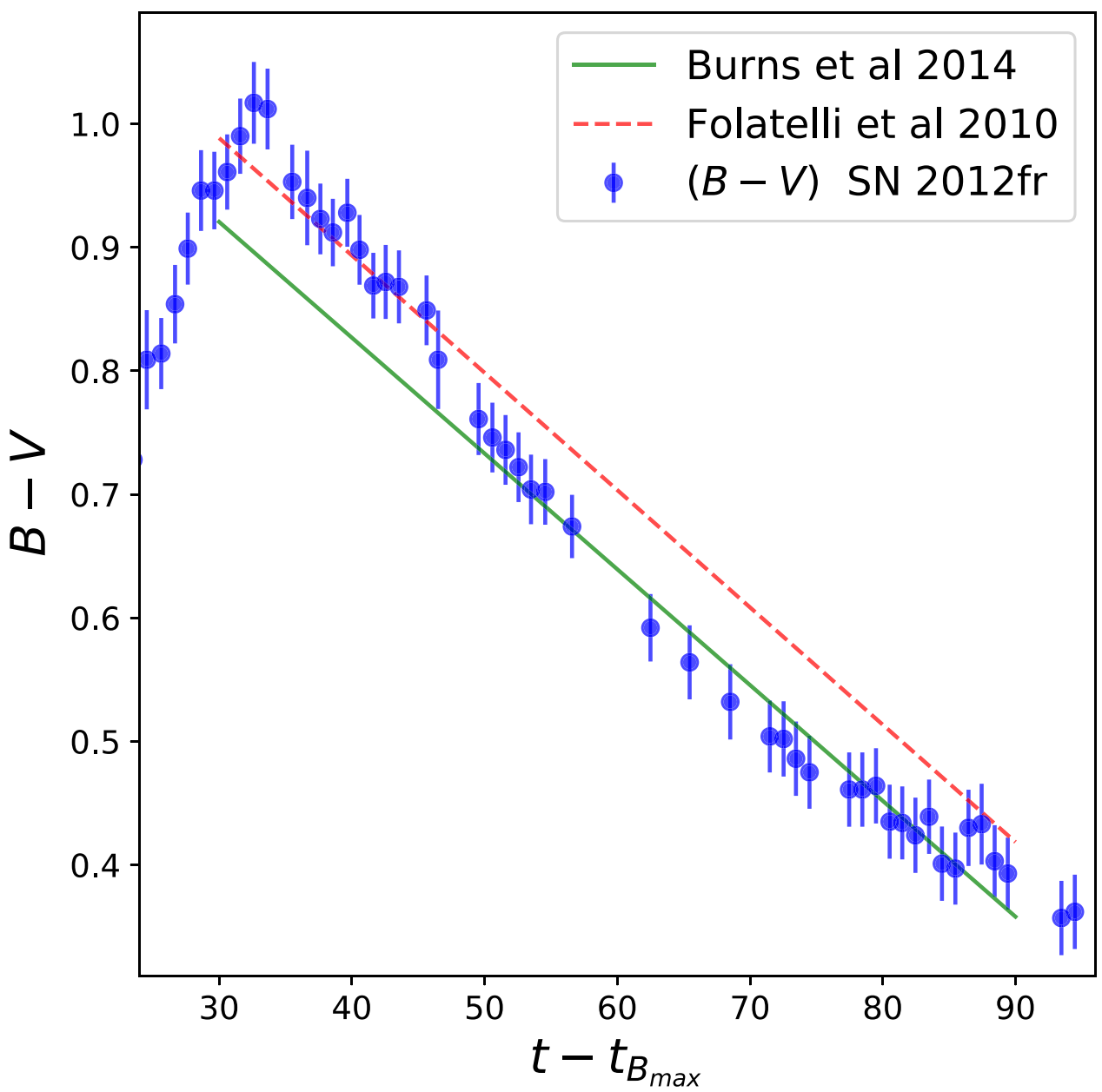

Figure 7. Galactic extinction-corrected $(B-V)$ color evolution of SN 2012fr. The Lira relation as determined by Folatelli et al. (2010) is over-plotted as a dashed line while the solid line is the recalibration presented by Burns et al. (2014). In both cases, if the overall range of the Lira relation is considered, then the implication is that SN $2012 \mathrm{fr}$ suffered little or no host-galaxy reddening.

\subsection{Bolometric Light Curve of SN $2012 \mathrm{fr}$}

The extended wavelength coverage of SN $2012 \mathrm{fr}$ afforded by observations spanning from UV through NIR wavelengths allows us to construct an essentially complete ultravioletoptical-infrared bolometric light curve, which is usually termed as UVOIR bolometric curve in the literature. However, as pointed out by Brown et al. (2016), this does not clearly specifiy the boundaries of the wavelength domain over which the flux estimate is done. Consequently, in this paper we define the bolometric luminosity, $L_{\mathrm{Bol}}$, as the luminosity between wavelengths of $1800 \AA$ and infinity, corresponding to the sum of $L_{\text {UVOIR }}(3000-16600 \AA)$ flux plus the contribution, $L_{\text {uvm } 2}(1800-3000 \AA)$, as deduced from the SWIFT photometry and the unobserved far-infrared $L_{\lambda>\lambda_{H}}(16600-\infty \AA)$.

Details of this calculation are given in Appendix B and the final bolometric light curve is plotted in Figure 8. The bolometric light curve derived by Pereira et al. (2013) for SN 2011fe is shown for comparison, which is one of the few other SNe Ia to have been well-observed in UV, optical, and NIR.

At peak, SN 2012fr reached a maximum luminosity of $L_{\mathrm{Bol}}=(1.35 \pm 0.14) \times 10^{43} \mathrm{erg} \mathrm{s}^{-1}$, which is on the bright end of the normal SNe Ia distribution (cf. Figure 6 of Scalzo et al. 2014). The relative fractions of the NIR $(\lambda>10000 \AA)$ integrated flux are plotted as a function of light-curve phase in
Figure 9, showing that the NIR contribution to the bolometric light curve of SN $2012 \mathrm{fr}$ is nearly $15 \%$ at -12 days. It then falls to a minimum of $\sim 4 \%$ a few days after $t_{B_{\max }}$ and rises again steeply to $19 \%$ at +40 days, at which point it begins to slowly decrease again. The latter behavior is similar to that shown by Scalzo et al. (2014) in Figure 3 and it is responsible for the prominent shoulder in the light curve that can be seen between +20 and +45 days in Figure 8. Figure 9 shows that the UV contribution $\left(\lambda<\lambda_{u}\right)$ to the bolometric light curve of SN 2012fr is generally of less importance than the NIR. The only exception is around the $t_{B_{\max }}$ epoch, when both contributions are similar, peak around $10 \%$ a few days before $t_{B_{\max }}$ and contribute $>5 \%$ of the integrated flux only during the early epochs; i.e., before 20 days after $t_{B_{\max }}$. This behavior is consistent with previous attempts to quantify the UV contribution to the bolometric luminosity (e.g., see Suntzeff 2003).

\subsection{Rise Time}

SN 2012fr was discovered by the TAROT collaboration on 2012 October 27.05 (UT), 15.6 days before $t_{B_{\max }}$. As reported by Klotz (2012), the SN was not visible to a limiting magnitude of $R>15.8$ in an image of NGC 1365 that was taken three days earlier on 2012 October 24.05 (UT) with the same telescope. A more stringent non-detection of $R>19.3$ on 2012 October 24.02 (UT) was obtained by J. Normand from 
Table 8

$\operatorname{gr}_{\text {LSQ }}$ Light Curve of SN 2012fr

\begin{tabular}{|c|c|c|c|}
\hline JD (days) & Phase $^{\mathrm{a}}$ (days) & $\mathrm{gr}_{\mathrm{LSQ}}(\mathrm{mag})$ & $\begin{array}{l}\text { Filter of } \\
\text { Observation }\end{array}$ \\
\hline 2456225.72 & -17.41 & $>20.38^{b}$ & $\mathrm{gr}_{\mathrm{LSQ}}$ \\
\hline 2456225.84 & -17.33 & $>20.34^{\mathrm{b}}$ & $\mathrm{gr}_{\mathrm{LSQ}}$ \\
\hline 2456226.69 & -16.41 & $17.71 \pm 0.03$ & $\mathrm{gr}_{\mathrm{LSQ}}$ \\
\hline 2456226.77 & -16.33 & $17.36 \pm 0.02$ & $\mathrm{gr}_{\mathrm{LSQ}}$ \\
\hline 2456227.55 & -15.55 & $16.03_{-0.10}^{+0.21}$ & open $_{\text {TAROT }}$ \\
\hline 2456227.62 & -15.48 & $16.24 \pm 0.06$ & $L_{\text {Slooh }}$ \\
\hline 2456228.86 & -14.24 & $15.21 \pm 0.03$ & open $_{\text {Boss }}$ \\
\hline 2456229.63 & -13.47 & $14.66 \pm 0.03$ & $V_{\text {TAROT }}$ \\
\hline 2456229.67 & -13.43 & $14.56 \pm 0.03$ & $V_{\text {TAROT }}$ \\
\hline 2456229.72 & -13.38 & $14.56 \pm 0.03$ & $V_{\text {TAROT }}$ \\
\hline 2456229.76 & -13.34 & $14.50 \pm 0.03$ & $V_{\text {TAROT }}$ \\
\hline 2456229.80 & -13.30 & $14.52 \pm 0.03$ & $V_{\text {TAROT }}$ \\
\hline 2456229.91 & -13.20 & $14.41 \pm 0.01$ & open $_{\text {Boss }}$ \\
\hline 2456230.63 & -12.47 & $13.99 \pm 0.01$ & $V_{\text {TAROT }}$ \\
\hline 2456230.67 & -12.43 & $13.94 \pm 0.02$ & $V_{\text {TAROT }}$ \\
\hline 2456230.70 & -12.40 & $13.95 \pm 0.01$ & $V_{\mathrm{CSP}}$ \\
\hline 2456230.72 & -12.38 & $13.89 \pm 0.01$ & $V_{\text {TAROT }}$ \\
\hline 2456230.76 & -12.34 & $13.91 \pm 0.03$ & $V_{\text {TAROT }}$ \\
\hline 2456230.80 & -12.30 & $13.87 \pm 0.01$ & $V_{\text {TAROT }}$ \\
\hline 2456230.94 & -12.16 & $13.79 \pm 0.02$ & open $_{\text {Boss }}$ \\
\hline 2456231.75 & -11.35 & $13.49 \pm 0.01$ & $V_{\mathrm{CSP}}$ \\
\hline 2456231.80 & -11.30 & $13.45 \pm 0.01$ & $V_{\text {TAROT }}$ \\
\hline 2456232.63 & -10.47 & $13.18 \pm 0.01$ & $V_{\text {TAROT }}$ \\
\hline 2456232.67 & -10.43 & $13.13 \pm 0.01$ & $V_{\text {TAROT }}$ \\
\hline 2456232.72 & -10.38 & $13.12 \pm 0.01$ & $V_{\text {TAROT }}$ \\
\hline 2456232.75 & -10.35 & $13.14 \pm 0.01$ & $V_{\mathrm{CSP}}$ \\
\hline 2456232.76 & -10.34 & $13.11 \pm 0.01$ & $V_{\text {TAROT }}$ \\
\hline 2456233.70 & -9.40 & $12.90 \pm 0.01$ & $V_{\mathrm{CSP}}$ \\
\hline 2456234.63 & -8.47 & $12.69 \pm 0.01$ & $V_{\text {TAROT }}$ \\
\hline 2456234.67 & -8.43 & $12.66 \pm 0.01$ & $V_{\text {TAROT }}$ \\
\hline 2456234.70 & -8.40 & $12.68 \pm 0.01$ & $V_{\mathrm{CSP}}$ \\
\hline 2456234.72 & -8.38 & $12.64 \pm 0.01$ & $V_{\text {TAROT }}$ \\
\hline 2456234.76 & -8.34 & $12.64 \pm 0.01$ & $V_{\text {TAROT }}$ \\
\hline 2456235.67 & -7.43 & $12.64 \pm 0.01$ & $V_{\mathrm{CSP}}$ \\
\hline 2456236.68 & -6.42 & $12.39 \pm 0.01$ & $V_{\mathrm{CSP}}$ \\
\hline 2456238.72 & -4.38 & $12.18 \pm 0.01$ & $V_{\mathrm{CSP}}$ \\
\hline 2456239.67 & -3.43 & $12.12 \pm 0.01$ & $V_{\mathrm{CSP}}$ \\
\hline 2456241.73 & -1.37 & $12.05 \pm 0.01$ & $V_{\mathrm{CSP}}$ \\
\hline 2456242.64 & -0.46 & $12.02 \pm 0.01$ & $V_{\mathrm{CSP}}$ \\
\hline 2456243.62 & +0.52 & $12.01 \pm 0.01$ & $V_{\mathrm{CSP}}$ \\
\hline 2456244.72 & +1.62 & $12.00 \pm 0.01$ & $V_{\mathrm{CSP}}$ \\
\hline 2456245.61 & +2.51 & $11.99 \pm 0.01$ & $V_{\mathrm{CSP}}$ \\
\hline 2456246.71 & +3.61 & $12.01 \pm 0.01$ & $V_{\mathrm{CSP}}$ \\
\hline 2456247.65 & +4.55 & $12.03 \pm 0.01$ & $V_{\mathrm{CSP}}$ \\
\hline 2456248.71 & +5.61 & $12.06 \pm 0.01$ & $V_{\mathrm{CSP}}$ \\
\hline
\end{tabular}

Notes.

a This phase was computed relative to $t_{B_{\max }}=$ JD 2456243.1.

b $3 \sigma$ upper limit for nondetection.

stacked images taken with an $0.6 \mathrm{~m}$ telescope at the Observatoire des Makes (Klotz 2012). Thus, the rise time to $t_{B_{\max }}$ was constrained to somewhere between 18.6 and 15.6 days.

Fortunately, the LSQ images that are presented in this paper provide a much tighter constraint on the rise time because the SN was clearly visible on 2012 October 26.19 (UT) but was absent in an image of similar depth that was obtained on 2012 October 25.34 (see Figure 4). Thus, the SN was detected less than a day after the explosion, which occurred some time between 17.3 and 16.5 days before $t_{B_{\max }}$.
Our observations of SN 2012fr present a rare opportunity to study the early rising light curve of SN 2012fr. However, we first must $\mathrm{S}$-correct the various measurements to the same filter bandpass. Given that the earliest detection and non-detection of SN 2012fr were made in the LSQ gr filter, we choose to convert the Slooh, TAROT, BOSS, and CSP photometry to LSQ gr magnitudes. The steps that are required are:

\section{Slooh L filter.}

As discussed in Appendix A, the Slooh L-band photometry is essentially in the same natural system as the $\mathrm{gr}_{\mathrm{LSQ}}$ observations. Hence, no S-correction is required for this measurement, which was obtained less than two hours after the TAROT discovery image.

\section{TAROT open filter.}

The TAROT discovery image was obtained within approximately 1-2 days of explosion. This is more than a day before the first spectroscopic observation. Fortunately, the Slooh $B$ and $G$ filter observations, which were obtained less than two hours after the TAROT discovery image, provide color information that can be used to estimate the S-correction under the assumption that the spectrum at this epoch can be approximated by a blackbody. Figure 10 shows the magnitude difference $\left(g r_{\mathrm{LSQ}}-\right.$ open $\left._{\mathrm{TAROT}}\right)$ as a function of $(B-G)_{\text {Slooh }}$ as derived from synthetic photometry of main-sequence stars from the Pickles (1998) stellar library spectra and black bodies of varying temperature. From the photometry given in Appendix A, $(B-G)_{\text {Slooh }}=$ $0.28 \pm 0.31 \mathrm{mag}$. Using the blackbody curve, this implies $\left(g r_{\mathrm{LSQ}}-\right.$ open $\left._{\mathrm{TAROT}}\right)=0.09_{-0.08}^{+0.20}$ mag.

As a sanity check on this result, we plot the $(B-V)$ color evolution for SN 2012fr in Figure 11. For comparison, observations of SN $2011 \mathrm{fe}$ are also shown. Combining the $B_{\text {Slooh }}$ and $G_{\text {Slooh }}$ magnitudes measured for the SN and the color-color plots in Appendix A gives $(B-V)=0.40 \pm 0.31 \mathrm{mag}$. This measurement is plotted in Figure 11 and it appears to be generally consistent with expectations if SN 2011fe is a valid comparison. Nevertheless, it should be kept in mind that the correction of the TAROT photometry to the LSQ system that was derived in the previous paragraph is strictly only valid for an object with a stellar or blackbody spectrum. Strong features such as the Ca II and Si II HVFs observed in the earliest spectra of SN 2012fr could affect the S-correction.

3. BOSS open filter.

The first BOSS open filter observation was made 14.2 days before $t_{B_{\max }}$. This is only $\sim 0.3$ days after the first spectrum of the SN obtained by Childress et al. (2012) and, therefore, we have used this spectrum (published by Childress et al. 2013) to calculate the S-correction. We find $\left(g r_{\mathrm{LSQ}}-\right.$ open $\left._{\mathrm{BOSS}}\right)=$ $-0.02 \pm 0.01 \mathrm{mag}$, where the error reflects the uncertainty in the spectrophotometric calibration of the spectrum. S-corrections for the BOSS open filter observations at -12.2 and -7.1 days were obtained from synthetic photometry of the Childress et al. (2013) spectra after color matching the spectra to the CSP photometry using first- or second- order polynomials (see Figure 12). Finally, the S-correction for the BOSS open filter observation at -13.2 days was obtained by interpolating the $\mathrm{S}$-corrections for the -14.2 and -12.2 day spectra. 


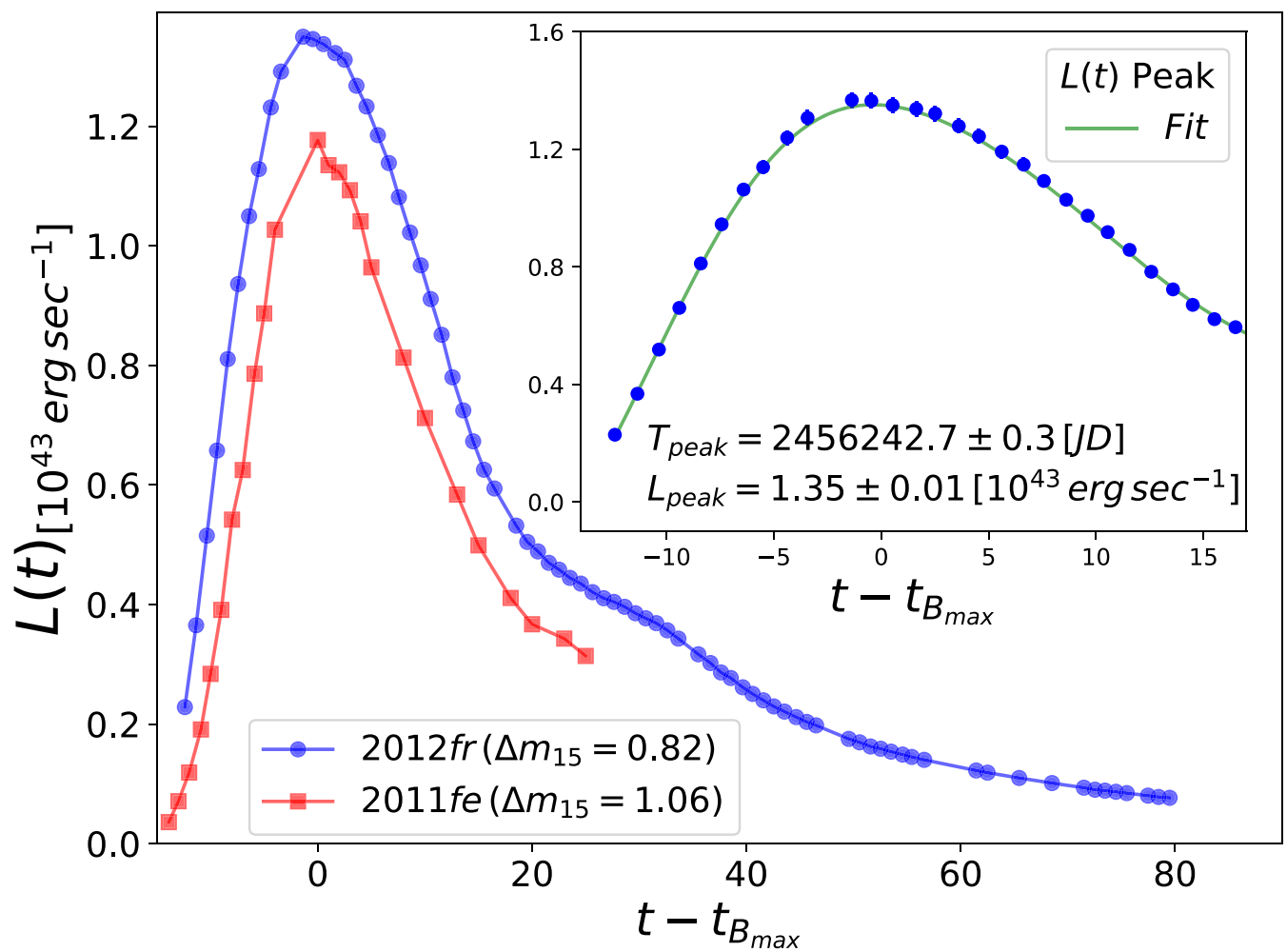

Figure 8. Bolometric light curve of SN 2012fr compared to that of the normal SN Ia 2011fe (Pereira et al. 2013). The bolometric light curve shown for SN 2012fr is the average of the trapezoidal rule integration and spectral template fitting methods (see Appendix B). Top right: a Gaussian process smooth curve is fitted to the bolometric data to recover the time and amplitude of peak.

\section{CSP and TAROT V filters.}

Although the $V$ filter is narrower than the LSQ gr filter, they are well-matched in central wavelength. The CSP $V$ observations began 12.4 days before $t_{B_{\max }}$ and the TAROT $V$ filter imaging started one night earlier at -13.5 days. S-corrections for both filters derived from the color-matched Childress et al. (2013) spectra are plotted in Figure 12.

The resulting LSQ gr light curve is given in Table 8 and it is plotted in normalized flux units in Figure 13. In addition, Figure 13 indicates the LSQ non-detections and the epoch of the first spectrum. From the first detection of the $\mathrm{SN}$ at -16.4 days with respect to $t_{B_{\max }}$ to the first BOSS observation at -14.2 days, the light curve rises very close to linearly. After the first BOSS observation, the light curve rises more steeply to a second nearly linear phase that lasts from approximately -11.5 to -6.5 days. The non-detection at -17.33 days and the first detection at -16.41 days constrain the time of explosion to have occurred at JD $2456226.23 \pm 0.46$, which is $16.87 \pm 0.46$ days before $t_{B_{\max }}$. The bolometric maximum was reached on JD $2456242.7 \pm 0.3$ (see Table 7). Hence, the rise to bolometric maximum took a total of $\sim 16.47 \pm 0.55$ days.

\section{6. ${ }^{56} \mathrm{Ni}$ Mass}

With a well-sampled bolometric light curve and a precise measurement of the bolometric rise time at hand, the amount of ${ }^{56} \mathrm{Ni}$ synthesized during the explosion can be estimated using Arnett's rule (Arnett 1982), which relates the bolometric rise time, $t_{r}$, and peak bolometric luminosity, $L_{\text {peak }}$, to the energy deposition, $E_{N i}$, within the expanding ejecta supplied by the radioactive decay chain ${ }^{56} \mathrm{Ni} \rightarrow{ }^{56} \mathrm{Co} \rightarrow{ }^{56} \mathrm{Fe}$ (see, e.g., Stritzinger \& Leibundgut 2005). This is formulated as follows:

$$
L_{\text {peak }}=\alpha E_{\mathrm{Ni}}\left(t_{r}\right) .
$$

Arnett's rule is derived from semi-analytical solutions to the radiative transfer problem of the expanding SN Ia ejecta. It explicitly assumes equality between energy generation and luminosity; i.e., the factor $\alpha=1$. A number of years ago, Branch (1992) surveyed the explosion models that were then available in the literature and concluded that $\alpha=1.2 \pm 0.2$ was a more appropriate value to use. While this value is still commonly employed for determining ${ }^{56} \mathrm{Ni}$ masses (see Scalzo et al. 2014, and the references therein), Höflich \& Khokhlov (1996) found that $\alpha$ ranged from 0.7 to 1.4 for a large variety of explosion models, with an average value of $1.0 \pm 0.2$. Meanwhile, Stritzinger \& Leibundgut (2005) cited radiative transport calculations for two modern 3D deflagration models from the MPA group that were both consistent with $\alpha=1.0$.

An expression for $E_{\mathrm{Ni}}\left(t_{r}\right)$ is provided by Nadyozhin (1994, see his Equation (18)). After plugging in various constants, this yields the following relation for $1 M_{\odot}$ of ${ }^{56} \mathrm{Ni}$ :

$$
\begin{aligned}
E_{\mathrm{Ni}}\left(1 M_{\odot}\right)= & {\left[6.45 \times e^{-t_{r} / 8.8}+1.45 \times e^{-t_{r} / 111.3}\right] } \\
& \times 10^{43} \mathrm{erg} \mathrm{s}^{-1} .
\end{aligned}
$$

By combining Equations (6) and (7), we obtain the following simple relation to estimate the ${ }^{56} \mathrm{Ni}$ mass:

$$
M_{\mathrm{Ni}}=\frac{L_{\text {peak }}}{\alpha E_{\mathrm{Ni}}\left(1 M_{\odot}\right)} M_{\odot} .
$$




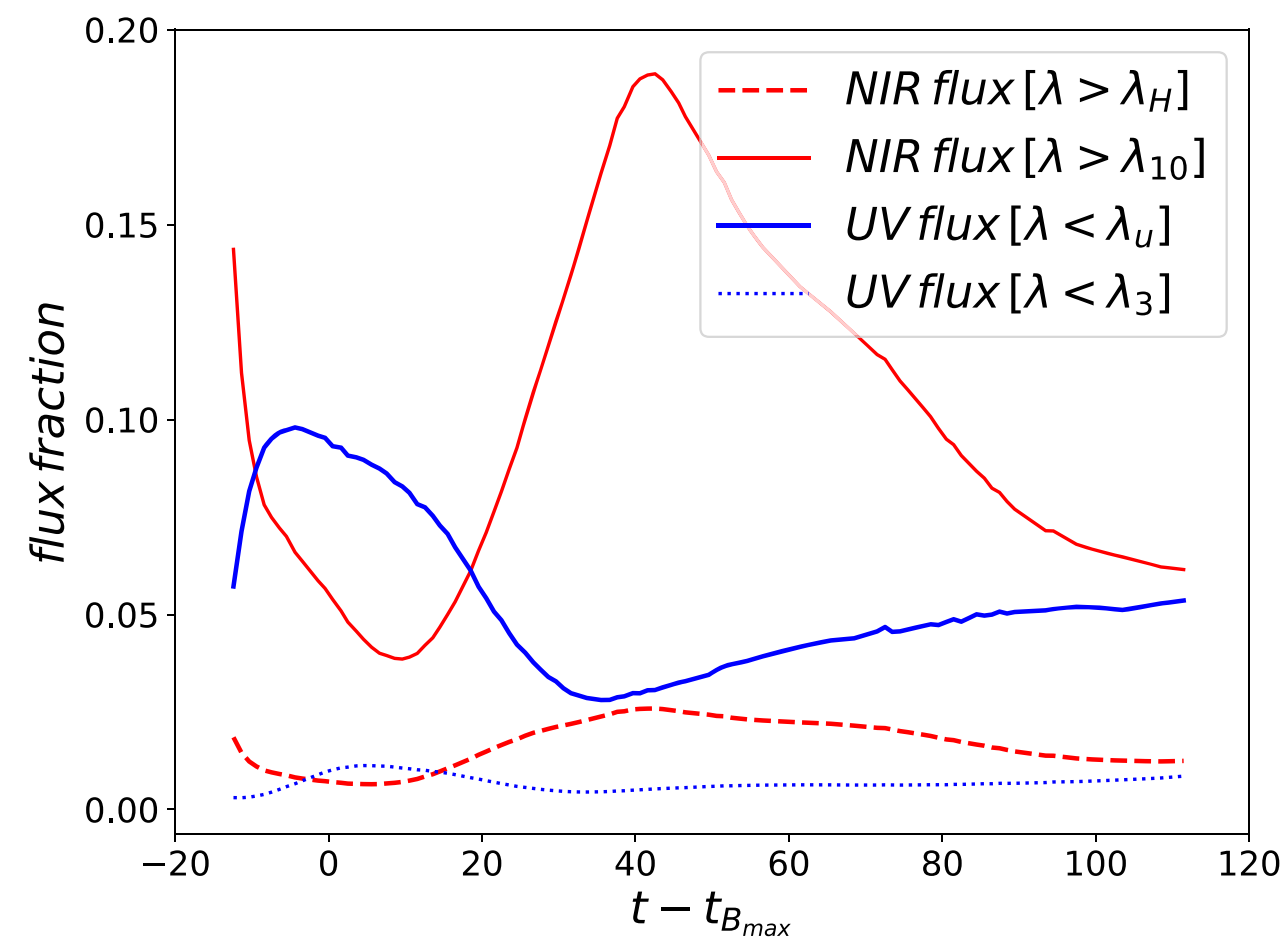

Figure 9. Estimated ratio of UV and NIR fluxes to the total flux for SN 2012fr. The dotted and dashed lines represent the ratios for estimated fluxes beyond the observed optical and NIR domain; i.e., fluxes for $\lambda$ less than $3000 \AA$ and for $\lambda$ greater than the effective wavelength of the $H$ band. For comparison purposes, the red solid line gives the flux beyond $10000 \AA$ and the blue solid line shows the UV flux for wavelengths less than $u$-band effective wavelength.

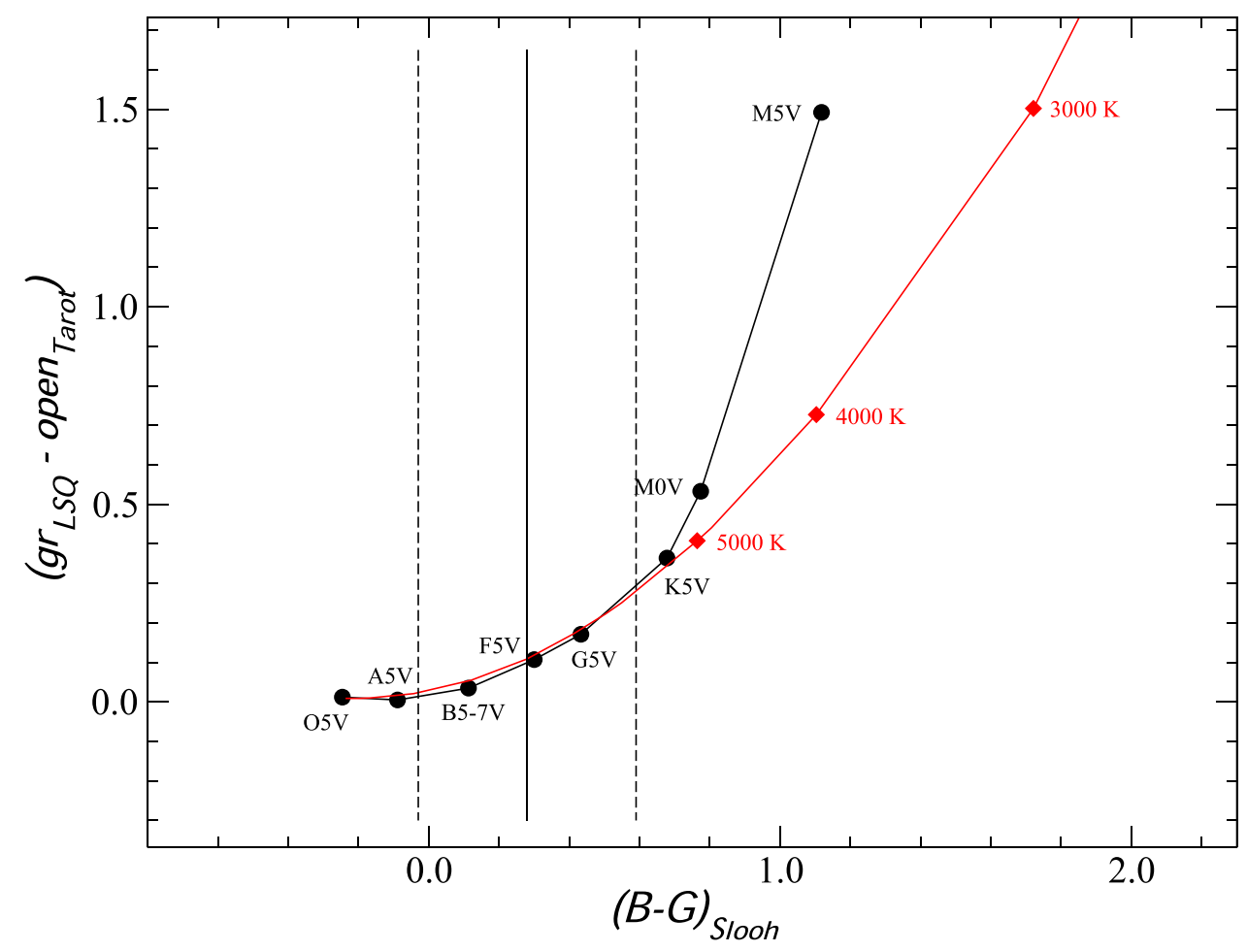

Figure 10. Color-color plot for the conversion of TAROT open filter magnitudes to the natural system magnitudes for the LSQ gr filter using the $(B-G)_{\text {Slooh }}$ measurement obtained less than two hours after the TAROT observation. The black curve show synthetic photometry carried out using the Pickles (1998) stellar atlas, and the red line corresponds to synthetic photometry of black bodies covering a range of temperature. The solid vertical line and the dashed lines on either side indicate the $(B-G)_{\text {Slooh }}$ color measurement of $0.28 \pm 0.31 \mathrm{mag}$.

Given the nonlinearity of Equation (8), we computed the error in $M_{N i}$ by simulating $10^{5}$ computations using randomly drawn uncorrelated values for $\alpha, E(B-V)_{\text {host }}, t_{r}$, the distance modulus, and the peak bolometric flux, $F_{\text {peak }}$, assuming that these parameters are described by Gaussian distributions with mean and standard deviation vectors of $\mu=[1.0,0.03,16.47$, 


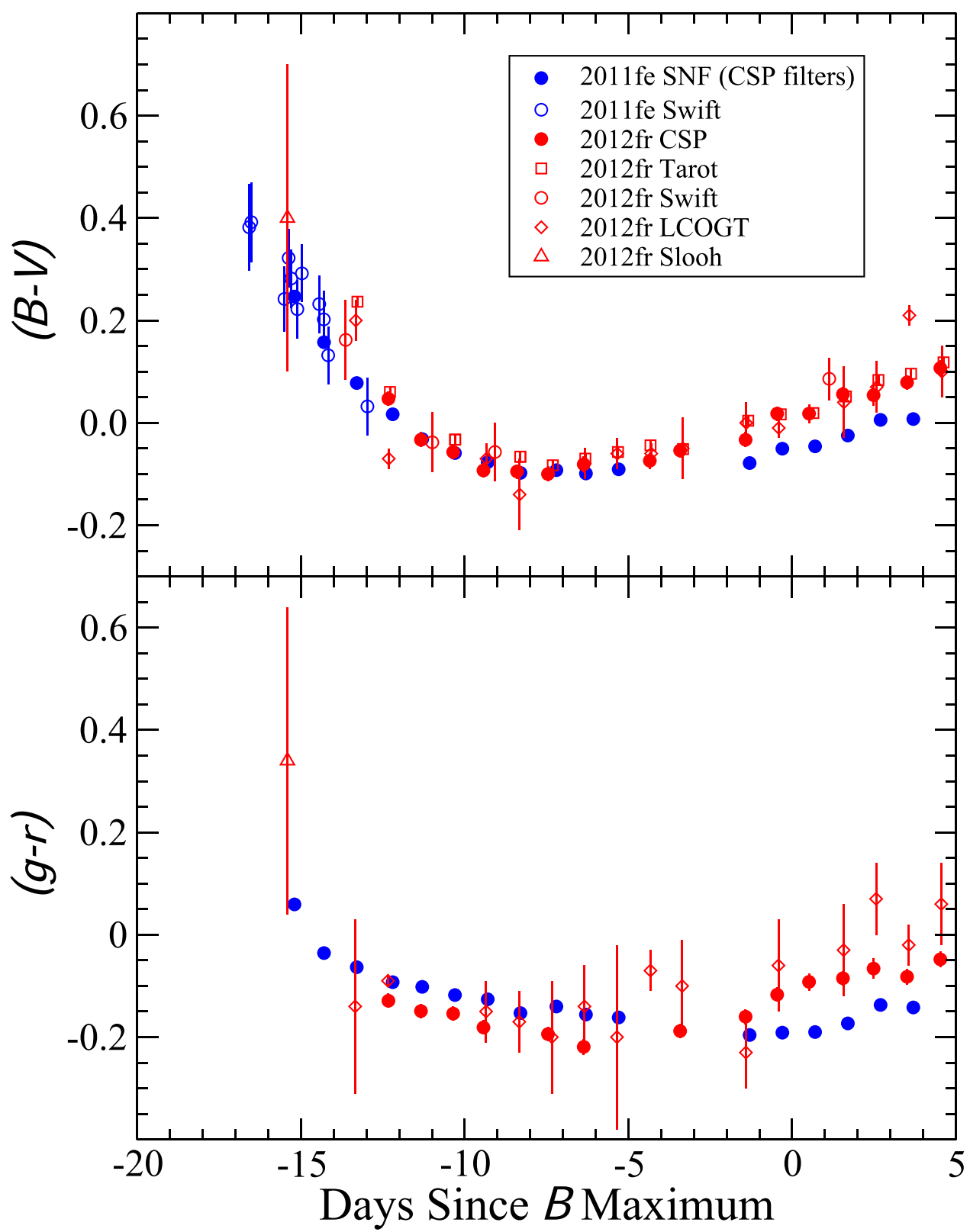

Figure 11. Early-time $(B-V)$ and $(g-r)$ color evolution of SN 2012fr. The color evolution of SN 2011fe is shown for comparison. The observations for SN 2012fr are taken from the CSP, Tarot, Swift, and LCOGT (Graham et al. 2017). The observations of SN 2011fe are from Swift (Brown et al. 2012) and synthetic photometry in the CSP filter bandpasses of the spectrophotometry of Pereira et al. (2013). The abscissa is corrected for time dilation.

$\left.31.27, F_{\text {peak }}\right]$ and $\sigma=\left[\begin{array}{lllll}0.2, & 0.03, & 0.60, & 0.05, & 0.05 F_{\text {peak }}\end{array}\right]$, respectively. Arnett's parameter, $\alpha$, the host-galaxy reddening, and the distance modulus are the dominant error sources, while the other parameters only exert a mild effect. The error in the host-galaxy reddening alone translates to an error of $10 \%$ in $M_{N i}$. The uncertainty in $\alpha$ has an even larger effect and introduces a slight asymmetry to the marginalized distribution of the ${ }^{56} \mathrm{Ni}$ mass. The final value derived from this analysis is $M_{\mathrm{Ni}}=0.60_{-0.14}^{+0.16} M_{\odot}$.

Childress et al. (2015) gave an independent estimate of $M_{N i}$ for SN 2012fr based on measurements of the [Co III] $\lambda 5893$ emission in nebular-phase spectra. Adjusting their value for the distance modulus for NGC 1365, which is adopted in the present paper, and assuming the same reddening of $E(B-V)_{\text {host }}=$ $0.03 \pm 0.03 \mathrm{mag}$ gives a value of $M_{\mathrm{Ni}}=0.61 \pm 0.07 M_{\odot}$. This is fully consistent with our estimate from the bolometric light curve.

We note that Zhang et al. (2014) derived a much higher ${ }^{56} \mathrm{Ni}$ mass from a bolometric light curve that was constructed from their own optical photometry, the same SWIFT ultraviolet observations used in the present paper, and NIR corrections taken from SN 2005cf (Wang et al. 2009). Adjusting their quoted value of $0.88 \pm 0.08 M_{\odot}$ to the same distance modulus and host-galaxy reddening that we assume in this paper gives a value of $0.84 \pm 0.08 M_{\odot}$. From J. Zhang (private communication), the peak bolometric luminosity of Zhang et al. (2014) was corrected to $L_{\text {peak }}=1.65 \times 10^{43} \mathrm{erg} \mathrm{s}^{-1}$. The mismatch is mainly due to an overestimation of the NIR contribution. After this correction and putting both measurements at the same distance modulus, the difference amounts to $7 \%$, half of which can be explained for our differences in the $u$-band domain flux. 


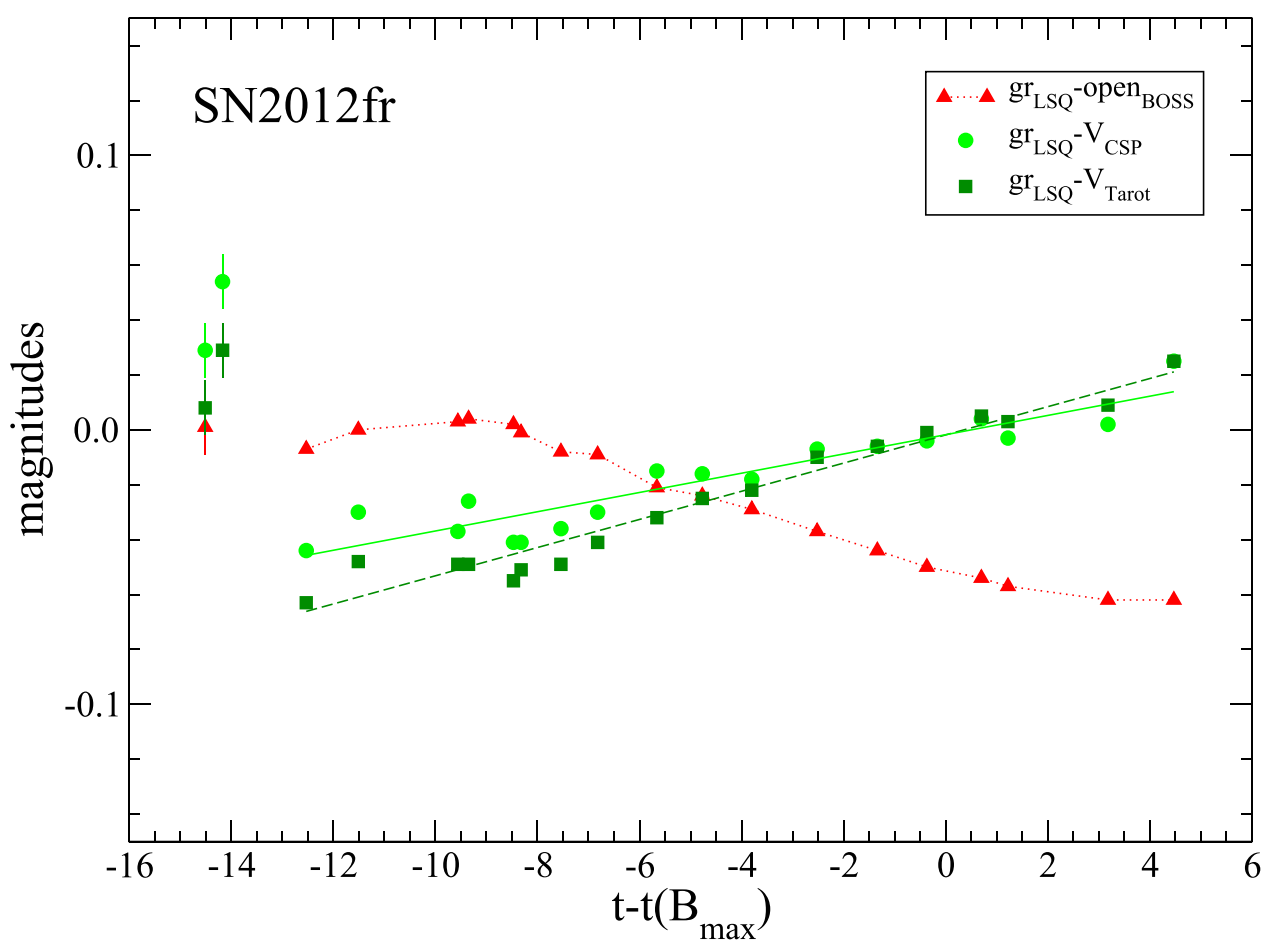

Figure 12. Magnitude differences between synthetic photometry of the Childress et al. (2013) spectra of SN 2012fr in the CSP $V$, TAROT $V$, and BOSS open filters and synthetic photometry of the same spectra in the LSQ gr filter. The spectra were first color matched to the CSP BVgri photometry to improve their spectrophotometric precision. The single BOSS point at -14.5 days was derived from the first spectrum obtained of the SN at -14.5 days (Childress et al. 2012). Likewise, the CSP $V$ and TAROT $V$ points at -14.5 and -14.2 days were calculated from the first two spectra obtained by (Childress et al. 2013). The larger errors on these points reflect the fact that these spectra could not be color matched because the CSP photometry did not begin until -12.4 days.

\section{Discussion}

\subsection{Early Light Curve}

The earliest emission of a SN Ia probes the location in the ejecta of the ${ }^{56} \mathrm{Ni}$ that powers the light curve and, therefore, is an important diagnostic of the explosion's physics (Piro \& Nakar 2013). Early studies of the rise times of SNe Ia (Riess et al. 1999; Aldering et al. 2000; Goldhaber et al. 2001; Conley et al. 2006; Hayden et al. 2010; Ganeshalingam et al. 2011; González-Gaitán et al. 2012) assumed or concluded that at the earliest epochs the flux increase is proportional to $t^{2}$. This socalled fireball model is physically motivated by the idea that the luminosity of a young SN Ia in a homologous expansion is most sensitive to its changing radius, and is less sensitive to changes in the temperature and photospheric velocity. More recently, Firth et al. (2015) also invoked a model with a single power law but they allowed the exponent to be a free parameter. In a variation of this, Shappee et al. (2016) also employed a power-law model but they allowed the exponent to be different for each filter.

Only recently have observations of individual SNe Ia allowed stringent tests of the validity of the power-law model. In two events, SNe 2011fe (Nugent et al. 2011) and 2012ht (Yamanaka et al. 2014), the fireball model (i.e., $n=2$ ) seems to provide a good fit to the earliest measurements. The most convincing of these two cases is SN 2011fe, which was discovered in M101 by the Palomar Transit Factory (PTF) with a $g$-band magnitude of 17.35 (Nugent et al. 2011). PTF images that were obtained in the previous night showed no source at a limiting magnitude of $g \geqslant 21.5$. Nugent et al. (2011) found that the fireball model provided a consistent fit to the first three nights of $g$-band measurements and they used it to infer a date of explosion only $11 \mathrm{hr}$ before discovery. However, as shown by Piro \& Nakar (2014), the bolometric light curve of SN 2011fe seems not to follow the fireball model.

Olling et al. (2015) presented observations of the light curves of three SN Ia that were followed nearly continuously by Kepler. The rising portion of the light curve of the brightest of these SNe, KSN 2011b, was found to be well-fitted by a single power-law but had an exponent of $n=2.44 \pm 0.14$.

Nevertheless, observations of SNe 2013dy (Zheng et al. 2013) and 2014J (Zheng et al. 2014; Goobar et al. 2015) have demonstrated quite clearly that the single power-law model does not apply to all SNe Ia. For both of these objects, highcadence (essentially daily) observations both before and after explosion revealed that the early-time light curves were well-described by a varying power-law exponent. The flux rose nearly linearly during the first day and then transitioned over the next 2-4 days to a relation closer to the $t^{2}$ law. Based on the results for these two events, Zheng et al. (2014) speculated that the varying power-law behavior may be common to SNe Ia and that previous results favoring the $t^{2}$ law may have been due to a lack of high-cadence observations constraining the shape of the light curve at the earliest epochs. This conclusion is supported by recently published observations of the early light curve of iPTF16abc (Miller et al. 2018), which also display a nearly linear rise during the first three days following explosion.

Figure 13 shows a comparison between our observations of the rising light curve of SN 2012fr with the broken power-law model used by Zheng et al. $(2013,2014)$ to fit the light curves of SNe 2013dy and 2014J. The time of first light for these fits has been adjusted to coincide with the value that we have determined for SN 2012fr. Note that for both of the latter SNe, 

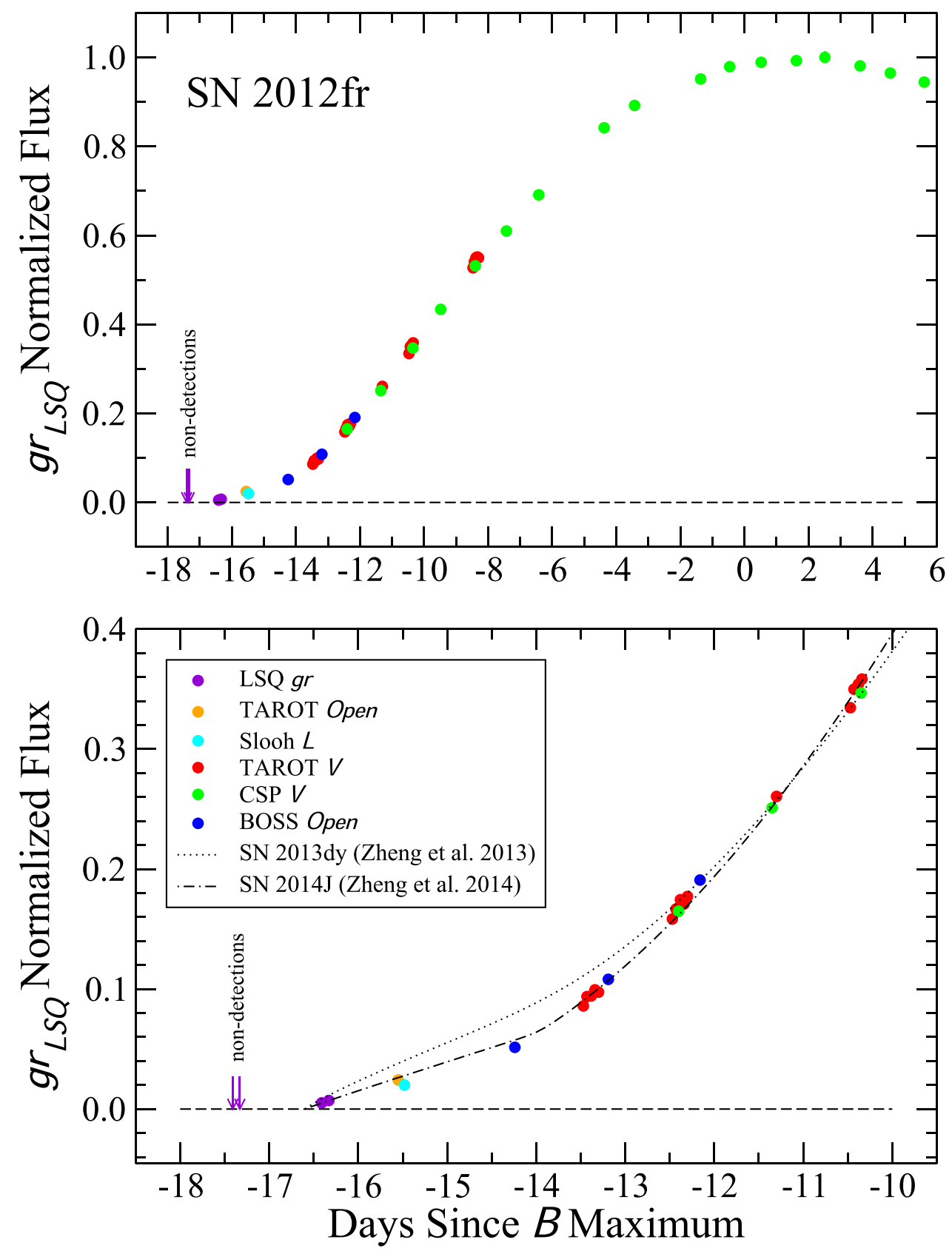

Figure 13. (Top) Relative fluxes (normalized to maximum) plotted as a function of the time with respect to $t_{B_{\max }}$ during the rising phase of the gr $\mathrm{LSQ}_{\mathrm{LS}}$-band light curve of SN 2012fr and extending to a few days past maximum. (Bottom) Enlargement of the same observations during the seven days following explosion. The error bars are not visible because they are the same size or smaller than the symbols that are used to plot the data. The broken power-law fits of the early light curves of SNe 2013dy and 2014J (Zheng et al. 2013, 2014) are plotted for comparison.

the fits were made to unfiltered photometry and, therefore, some caution should be taken in comparing these results with our $\mathrm{gr}_{\mathrm{LSQ}}$ light curve of SN 2012fr. Nevertheless, the resemblance is remarkable and supports the view that this behavior of a nearly linear rise initially, followed by a steeper increase in luminosity, may be common among SNe Ia.

Piro \& Morozova (2016) have investigated how the distribution of ${ }^{56} \mathrm{Ni}$ in the ejecta of a SN Ia affects the earliest phases of the light curve using SNEC (SuperNova Explosion Code; Morozova et al. 2015), which is an open source Lagrangian radiation hydrodynamics code that allowed them to initiate a shock wave within a white dwarf model, explode the white dwarf, and follow the early light curve evolution. These authors found that models with more highly mixed ${ }^{56} \mathrm{Ni}$ rise more quickly than do models with centrally concentrated ${ }^{56} \mathrm{Ni}$. Using a grid of 800 models generated with SNEC, we have attempted to match the early light curve of SN $2012 \mathrm{fr}$. A comparison of one of our better fitting light curves along with a range of other light curves with varying ${ }^{56} \mathrm{Ni}$ mixing is shown in Figure 14 (with the best-fit light curve in green). This demonstrates that the steepening of SN 2012fr's light curve over the first couple of days can naturally be accounted for by a moderately mixed ${ }^{56} \mathrm{Ni}$ distribution. This corresponds to a ${ }^{56} \mathrm{Ni}$ mass fraction of 0.05 at roughly $0.05 M_{\odot}$ below the surface of the exploding white dwarf. If ${ }^{56} \mathrm{Ni}$ were not mixed out to this shallow region, then the theoretical light curves would tend to 


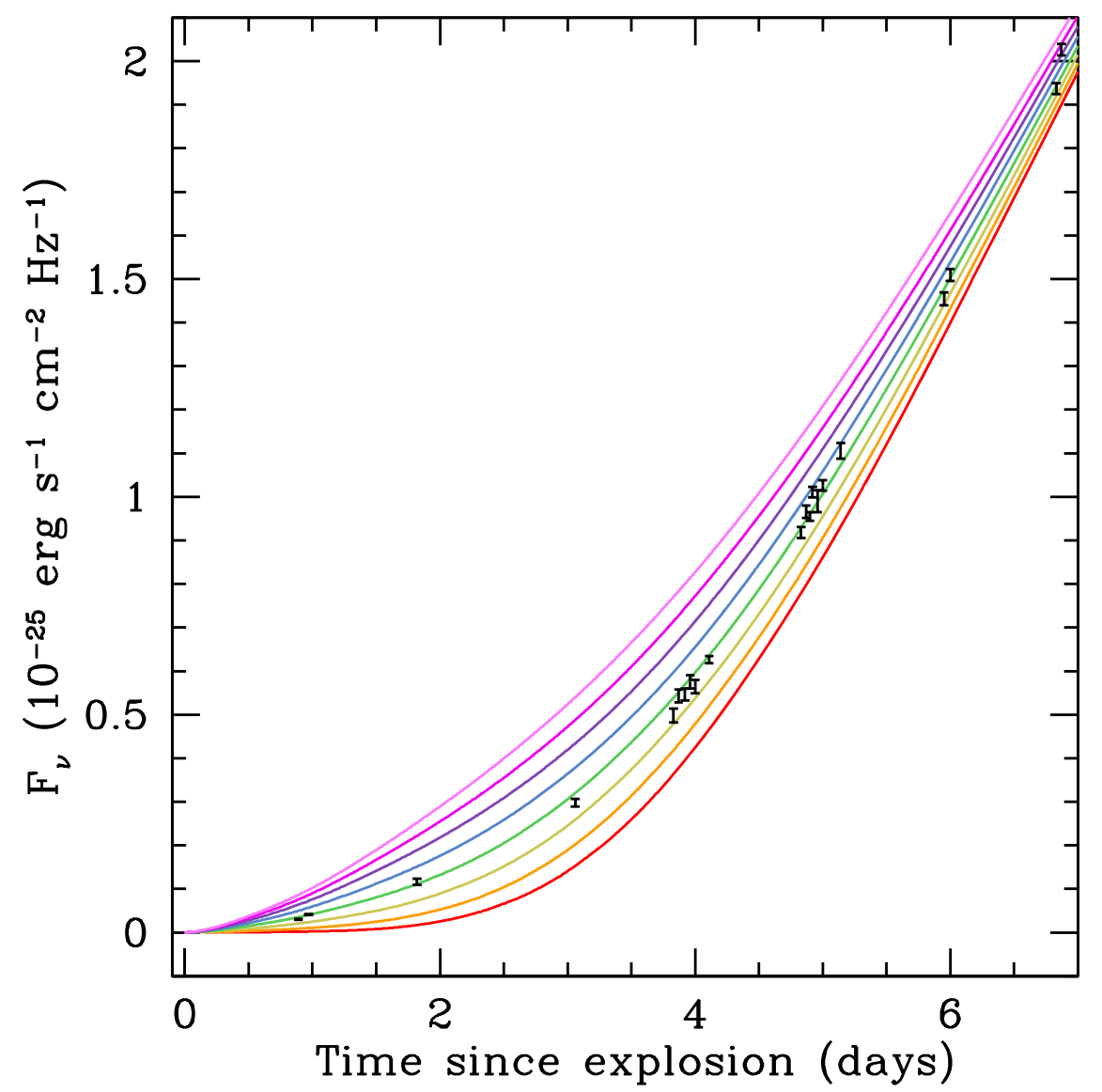

Figure 14. Observed flux in the LSQ gr-band as a function of time during the first seven days following explosion for a bare white dwarf model. The line colors indicate the level of ${ }^{56} \mathrm{Ni}$ mixing, which correspond to the profiles shown in Figure 15.

rise too slowly in comparison to SN 2012fr. Whereas, if the ${ }^{56} \mathrm{Ni}$ mixing were more strong, then we would not expect to see the change in slope in the early light curve.

This fit suggests that the first data point for this event was $\sim 21 \mathrm{hr}$ after explosion. Constraining the explosion time like this is important if we wish to be able to put limits on the interaction with a companion, as argued in Shappee et al. (2016). The corresponding ${ }^{56} \mathrm{Ni}$ mass fraction distributions for the models from Figure 14 are shown in Figure 15.

The observations only constrain the distribution of ${ }^{56} \mathrm{Ni}$ for material interior to the vertical dashed line. Constraints on shallower material would require even earlier observations. Whether or not the ${ }^{56} \mathrm{Ni}$ distribution we find for SN 2012fr is unique is unclear because degeneracies may arise between various physical parameters when only photometric data is considered (Noebauer et al. 2017). This point will be explored in more detail in a forthcoming work (A. L. Piro et al. 2017, in preparation). In the future, it will be useful to also have spectral information at these early epochs to uniquely determine the physical cause of the early light curve shape.

\subsection{Spectroscopic Peculiarities}

As more SNe Ia have been observed, several subclasses within the general phenomenon have been identified. Benetti et al. (2005) divided SNe Ia into three groups based on the light curve decline rate, $\Delta m_{15}(B)$, and the post-maximum evolution of the velocity of the minimum of the $\mathrm{Si}$ II $\lambda 6355$ absorption. The FAINT group, as represented by the prototypical SN 1991bg (Filippenko et al. 1992; Leibundgut et al. 1993;
Turatto et al. 1996), consists of fast-declining events $\left(\Delta m_{15}(B)>1.5 \mathrm{mag}\right)$ with low Si II velocities at maximum, which decrease rapidly with time. SNe Ia is then further split with normal decline rates $\left(\Delta m_{15}(B)<1.5 \mathrm{mag}\right)$ into two further categories: the HVG group, which display a high temporal velocity gradient $\left(\dot{v}>70 \mathrm{~km} \mathrm{~s}^{-1}\right.$ day $\left.^{-1}\right)$ in the days following $t_{B_{\max }}$; and the more common LVG events, which show a lower velocity gradient. The HVG events typically also have high Si II velocities at maximum. This led Wang et al. (2009) to propose a parallel subtype classification that was solely based on a measurement of the $\mathrm{Si}$ II $\lambda 6355$ velocity within a week of maximum. Wang et al. termed those having velocities $\gtrsim 11,800 \mathrm{~km} \mathrm{~s}^{-1}$ at the epoch of $t_{B_{\max }}$ high-velocity (HV) SNe Ia. Lower-velocity events, not including the 1991bglike and 1991T-like spectral types (Branch et al. 1993), were termed "normal" by Wang et al. As shown in Figure 2 of Foley et al. (2011) and Figure 6 of Silverman et al. (2012), 80\%$90 \%$ of HV events also pertain to the HVG group. Conversely, most LVG objects have normal Si II velocities in the Wang et al. classification system (Silverman et al. 2012). ${ }^{30}$

Branch et al. (2006, 2009) developed an independent classification system for SNe Ia that is based on a plot of the pseudo-equivalent widths of the Si II $\lambda 5972$ and $\lambda 6355$ absorption features. Four groups were established: Core Normal (CN), Cool (CL), Broad Line (BL), and Shallow Silicon (SS). Figure 16 displays these groups in a branch

\footnotetext{
$\overline{30}$ Note that the LVG subtype as defined by Benetti et al. includes 1991T-like objects.
} 


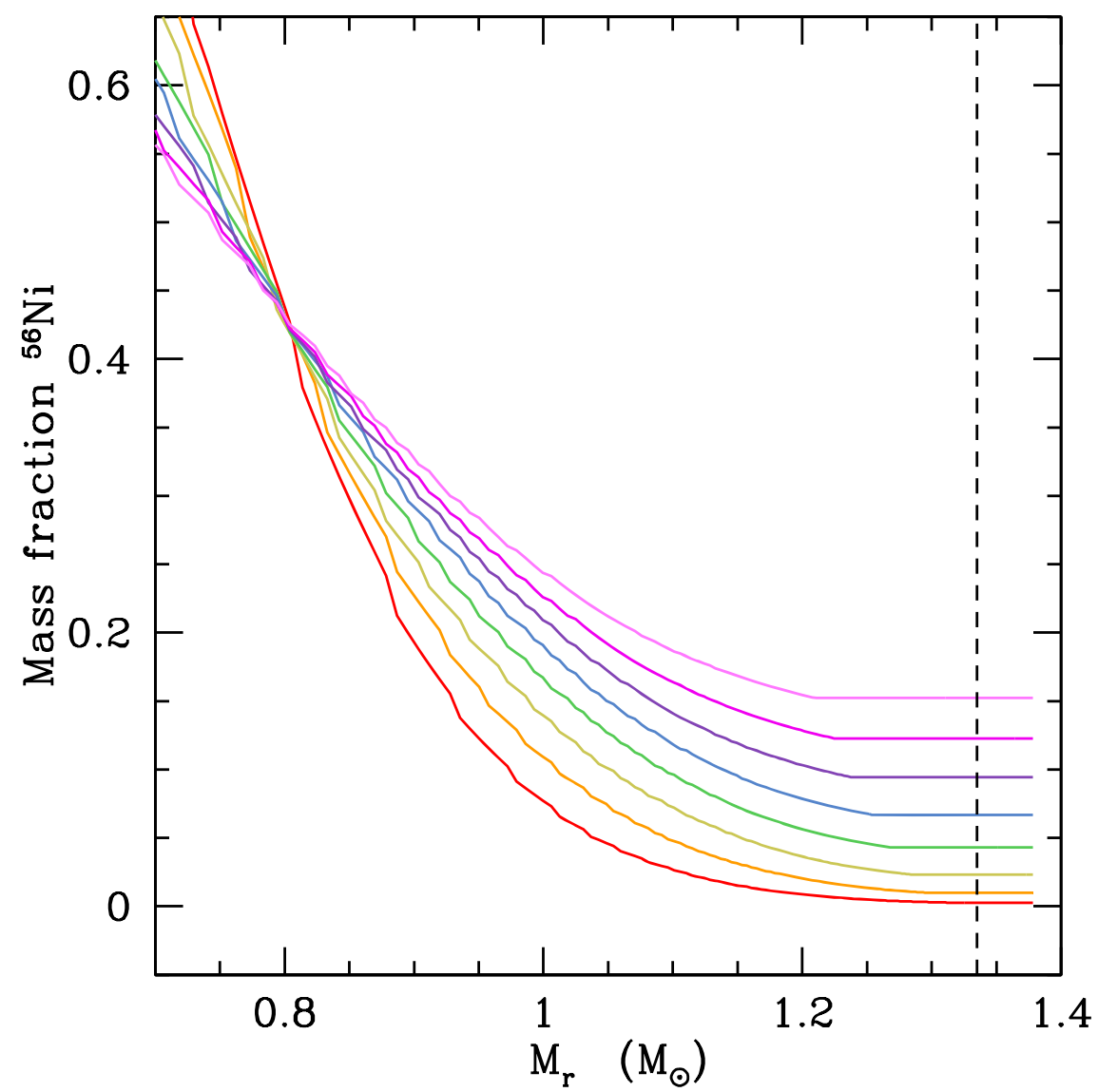

Figure 15. Profiles of the mass fraction ${ }^{56} \mathrm{Ni}$ as a function of the mass coordinate in the white dwarf for the various levels of mixing corresponding to the light curves shown in Figure 14. These are constrained by the observations only out to the vertical dashed line.

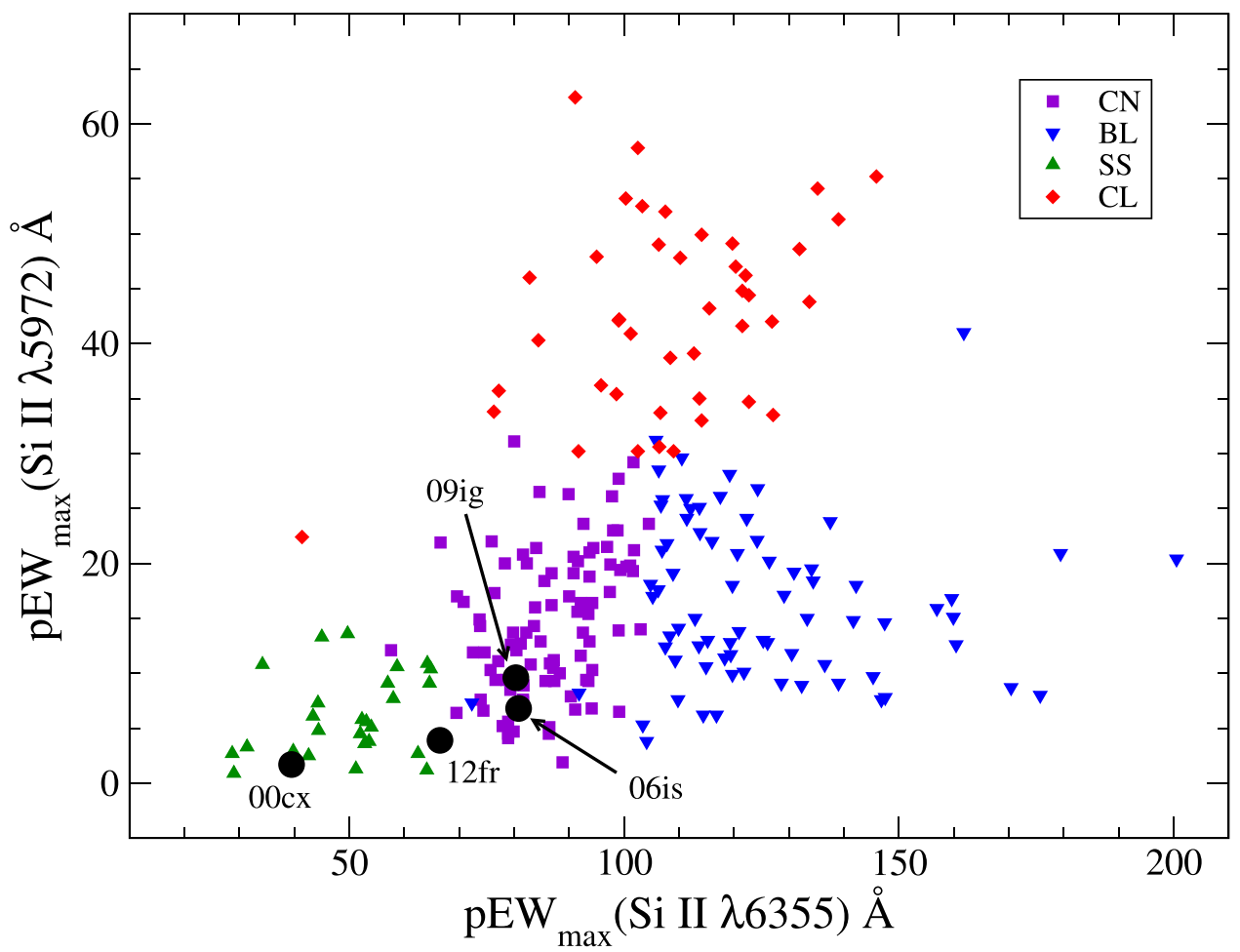

Figure 16. Plot of the pseudo-equivalent width of the Si II $\lambda 5972$ absorption vs. that of the Si II $\lambda 6355$ absorption within five days from maximum light, illustrating the four subtypes of SNe Ia defined by Branch et al. (2006). Core Normal (CN; violet squares), Shallow Silicon (SS; green upward-looking triangles), Broad Line (BL; blue downward-looking triangles), and Cool (CL; red diamonds) subtypes taken from the data of Blondin et al. (2012) are plotted. The positions of the shallow velocity gradient SNe 2000cx, 2006is, 2009ig, and 2012fr are indicated by black circles. 


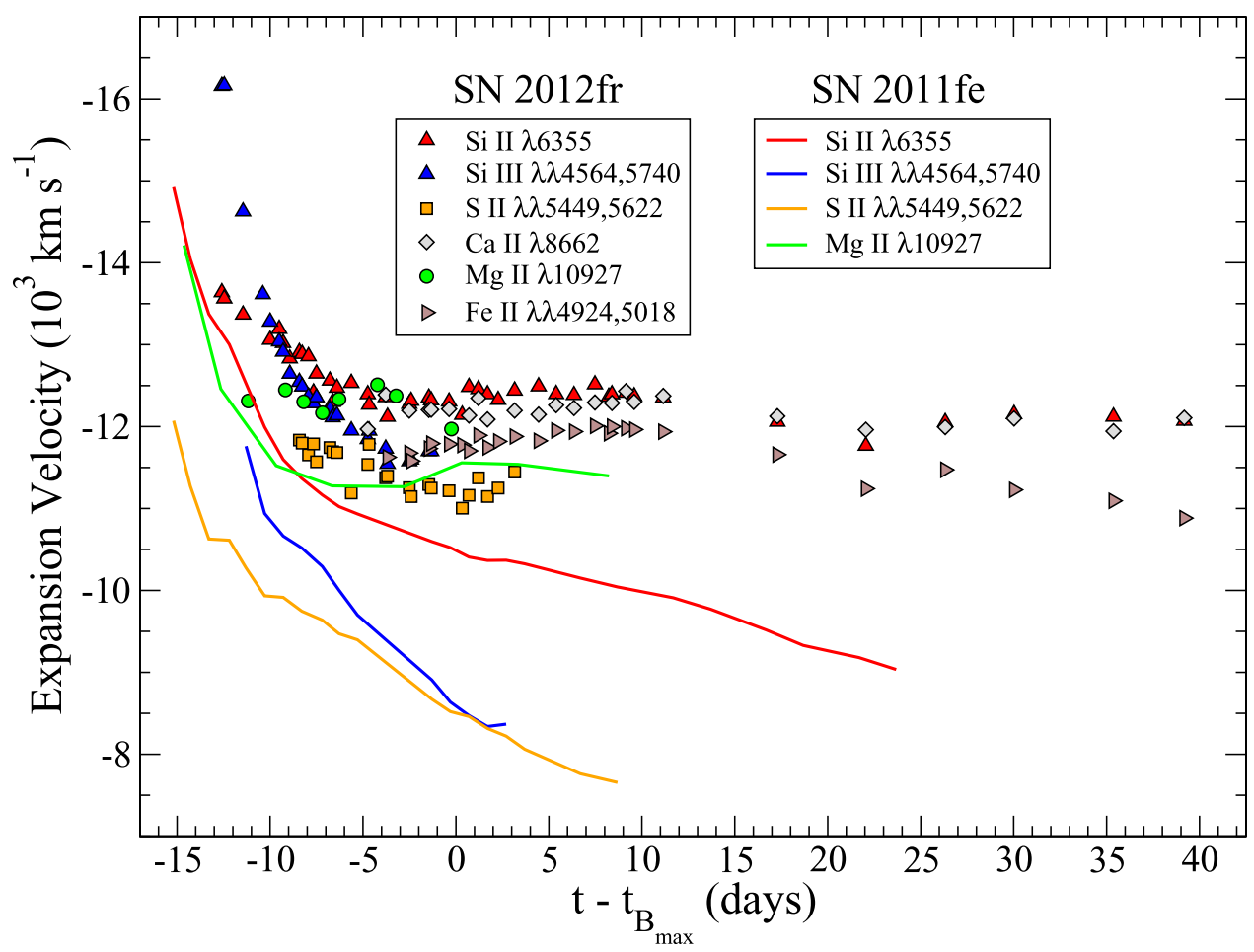

Figure 17. Comparison of the velocity stratification of different ions for SNe $2011 \mathrm{fe}$ and $2012 \mathrm{fr}$. The measurements correspond to the absorption minima of each line.

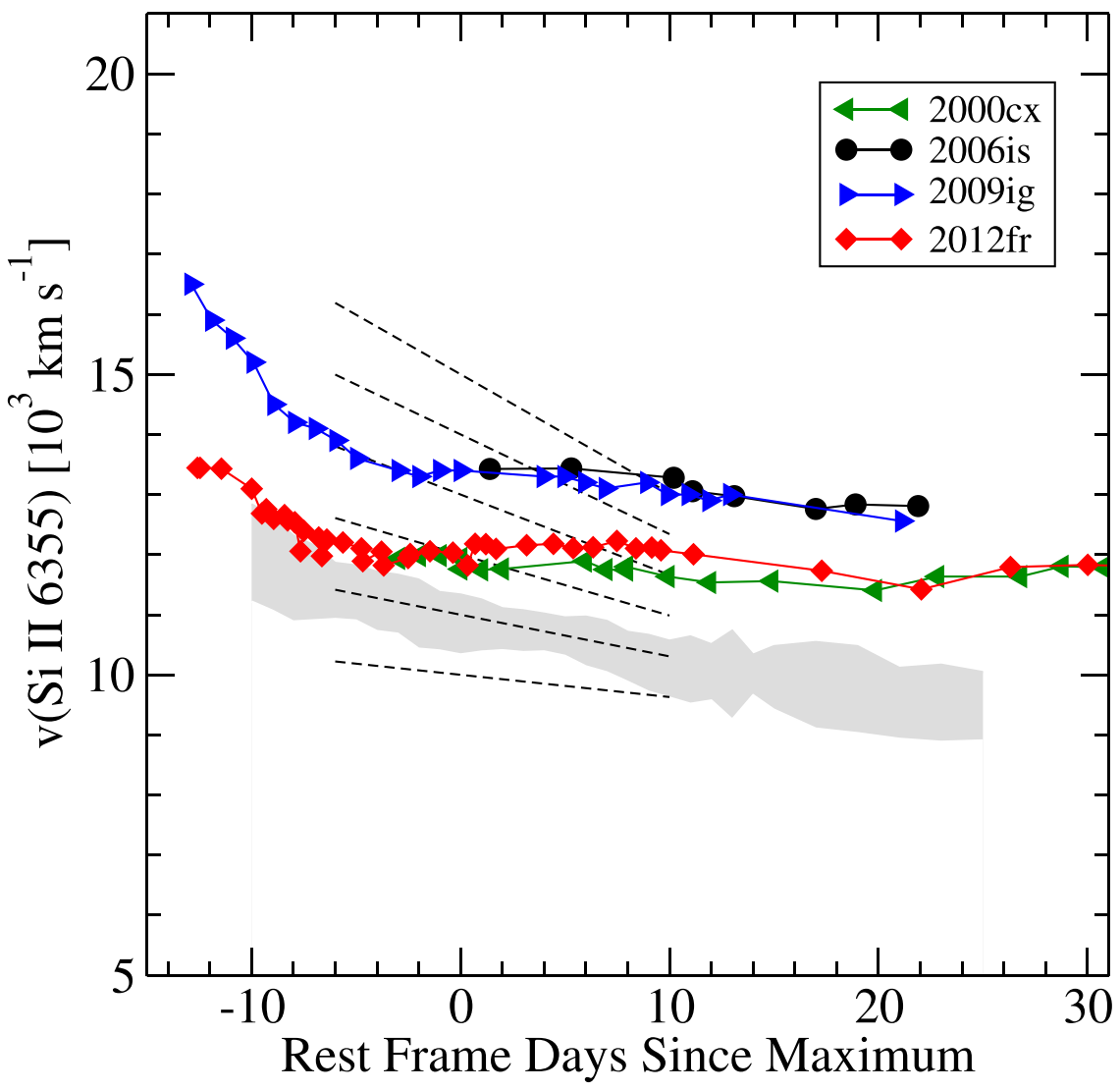

Figure 18. Evolution of the photospheric Si II $\lambda 6355$ velocity for the shallow velocity gradient SNe 2000cx, 2006is, 2009ig, and 2012fr. The shaded area shows the average and $1 \sigma$ dispersion of Si II velocities of normal SNe Ia reproduced from Folatelli et al. (2013), while the dashed lines represent a subset of the family of functions that describe the velocity evolution of normal and high-velocity gradient SNe Ia (Foley et al. 2011). The measurements for SNe 2000cx, 2009ig, and 2012fr are taken from Li et al. (2001), Marion et al. (2013), and Childress et al. (2013), while those for SN 2006is were made using the spectra of Folatelli et al. (2013). 


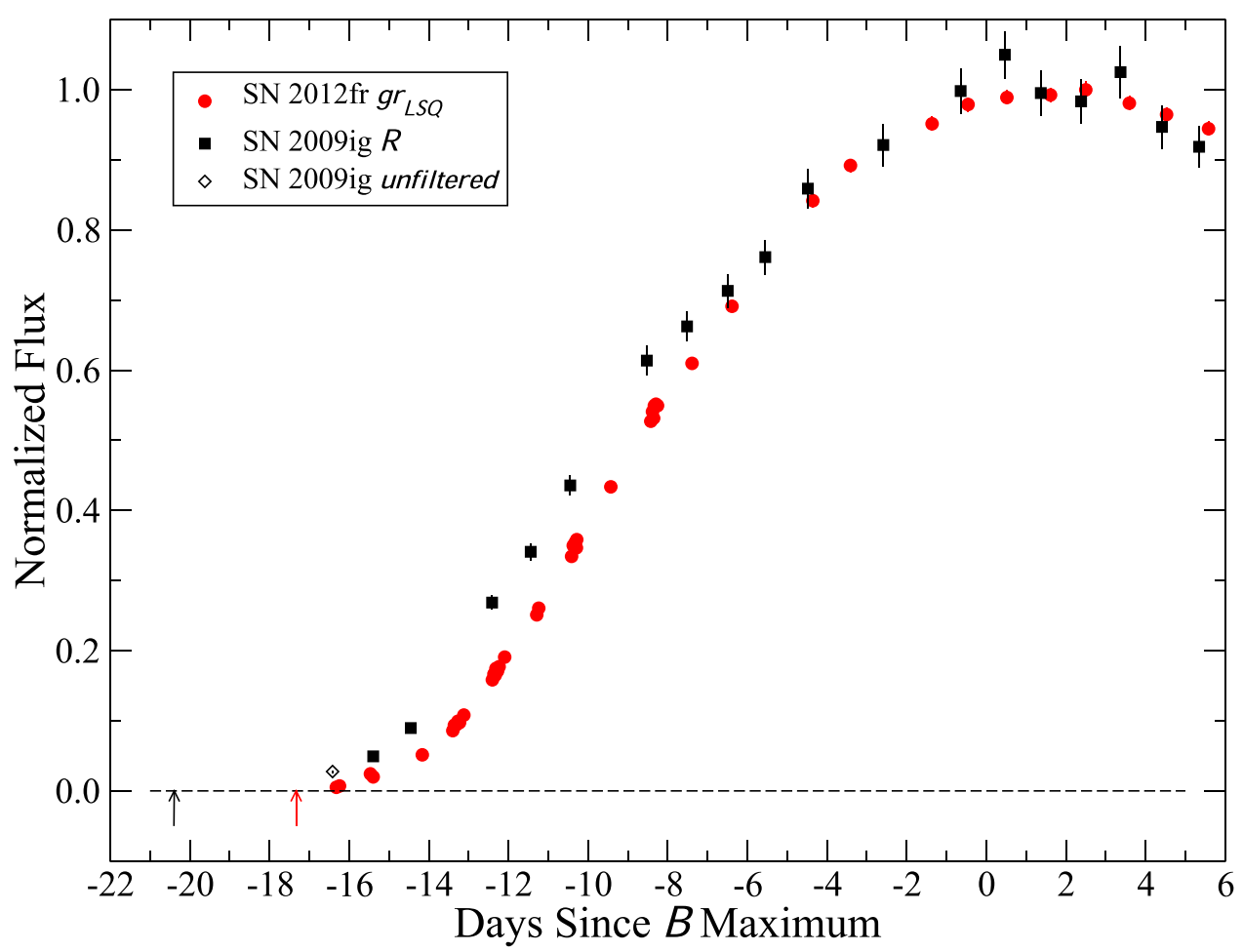

Figure 19. Relative fluxes (normalized to maximum) plotted as a function of the time with respect to $t_{B \max }$ for the $R$-band light curve of SN 2009 ig and the gr $r_{L S Q}$ light curve of SN 2012fr. The photometry for SN 2009ig is taken from (Foley et al. 2012). The unfiltered first observation was approximately S-corrected assuming a color $(B-V)=0.6 \pm 0.1$ and a sensitivity function similar to those of the Tarot and BOSS unfiltered photometry. The black arrow shows the last non-detection before discovery for SN 2009ig and the red arrow shows the same for SN 2012fr. The abscissa is corrected for time dilation.

diagram, using the data and classification criteria of Blondin et al. (2012). There is a rough correspondence between the BL and HV (or HVG) groups, with Blondin et al. (2012) and Folatelli et al. (2013) independently finding that two-thirds of $\mathrm{SNe}$ Ia that are classified as BL also belong to the HV subtype. The CL-type correlates well with the Benetti et al. FAINT and Wang et al. 1991bg groups, and the LVG (or Wang et al. normal) $\mathrm{SNe}$ generally encompass the $\mathrm{CN}$ and SS classes. Finally, the SS group includes not only 1991T-like SNe but also the peculiar 2002cx-like events (Li et al. 2003).

So, how does SN 2012fr fit into these classification schemes? Childress et al. (2013) found that SN 2012fr lies on the border separating the Branch et al. CN and SS subclasses (see Figure 16). The shallow SN 2012fr Si II velocity gradient displayed by SN 2012fr also places it clearly in the Benetti et al. LVG group. Nevertheless, the high Si II velocity at maximum of $\sim 12,000 \mathrm{~km} \mathrm{~s}^{-1}$ qualifies SN $2012 \mathrm{fr}$ as an $\mathrm{HV}$ event in the Wang et al. system. This is an unusual combination of classifications because $5 \%$ or less of SNe Ia in the $\mathrm{CN}+\mathrm{SS}$ groups belong to the HV subtype and $<10 \%$ of LVG SNe Ia are also classified as HV events (Blondin et al. 2012; Folatelli et al. 2013).

Figure 17 shows our measurements of the evolution of the expansion velocities of the Si II $\lambda 6355$, Si III $\lambda \lambda 4564,5740$, S II $\lambda \lambda 5449,5622$, Ca II $\lambda 8662$, and Fe II $\lambda \lambda 4924,5018$, absorption minima from the Childress et al. (2013) spectra of SN 2012fr. The expansion velocities of the Mg II $\lambda 10927$ line measured from unpublished CSP-II NIR spectra of SN 2012fr are also included. For comparison, we plot the expansion velocities of the same features as measured from the spectra of the prototypical SN 2011fe published by Pereira et al. (2013) and Hsiao et al. (2013). The small amount of velocity stratification of the intermediate mass elements (IMEs) in SN 2012fr from -5 days onward is remarkable and is in stark contrast to that observed for SN 2011fe. Childress et al. (2013) concluded that there is either a shell-like density enhancement in the ejecta at a velocity of $\sim 12,000 \mathrm{~km} \mathrm{~s}^{-1}$ or a sharp cutoff in the radial distribution of the IMEs in the ejecta. As discussed by Quimby et al. (2007) in the context of the slow-declining SN 2005hj-which is a Wang et al. (2009) normal event that showed a nearly flat Si II velocity gradient-a strong density enhancement is not predicted by standard delayed-detonation and deflagration models but is instead suggestive of rapidly expanding material interacting with overlying material. Examples of scenarios that produce a well-defined shell of IMEs include pulsating delayed-detonations (Khokhlov et al. 1993) or tamped detonations in a double-degenerate merger (Fryer et al. 2010). This possibility will be explored in more detail in a future paper (C. Cain et al. 2018, in preparation).

In the case of SN 2012fr, Childress et al. (2013) argued that it is more likely that the small velocity evolution of the Si II and Ca II lines reflects the fact that these ions are physically confined to a narrow region in velocity space in the ejecta. This conclusion was based, in part, on the observation that the expansion velocity of the Fe II lines in SN 2012fr began to slowly decrease $\sim 10$ days after maximum while the Si II and Ca II lines remained at a nearly constant velocity of $12,000 \mathrm{~km} \mathrm{~s}^{-1}$ (see Figure 17). One interpretation of Figure 17 is that the inner edge of the IMEs in the ejecta of SN $2012 \mathrm{fr}$ was located at a velocity of $\sim 11,000 \mathrm{~km} \mathrm{~s}^{-1}$, which 


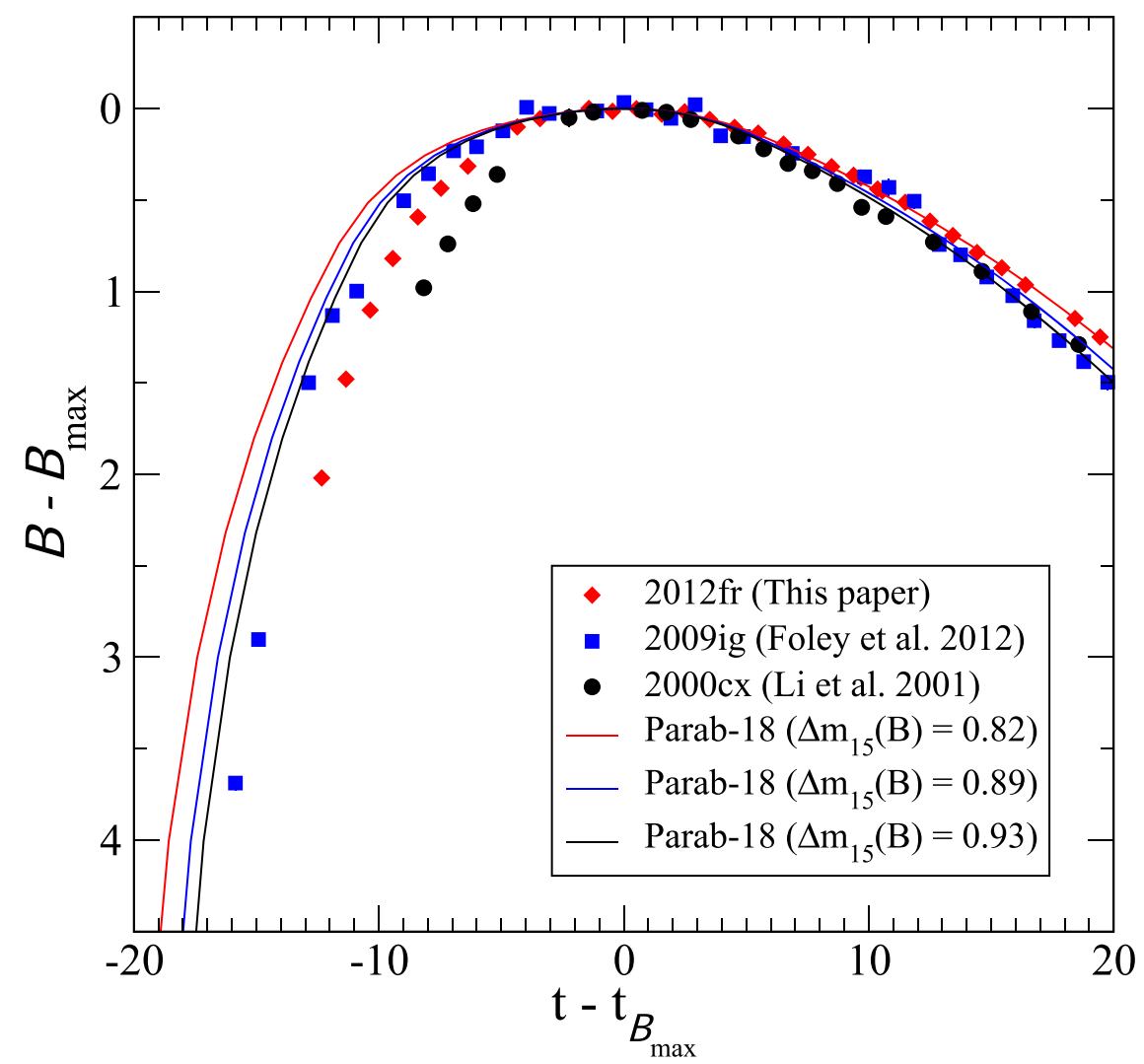

Figure 20. Comparison of $B$-band light curves for SN 2000cx $\left(\Delta m_{15}(B)=0.93 \mathrm{mag}\right)$, SN 2009ig $\left(\Delta m_{15}(B)=0.89 \mathrm{mag}\right)$, and SN $2012 \mathrm{fr}\left(\Delta m_{15}(B)=0.82 \mathrm{mag}\right)$. The abscissa is corrected for time dilation. Goldhaber et al.'s (2001) $B$-band template is shown for comparison, stretched to the decline rates of the three SNe.

is in contrast to a typical SN Ia as represented by $2011 \mathrm{fe}$ for which an abundance tomography analysis indicates that the inner edge of the IMEs extended down to $\sim 5000 \mathrm{~km} \mathrm{~s}^{-1}$ (Mazzali et al. 2015). This suggests a ${ }^{56} \mathrm{Ni}$ distribution that extended out to higher velocities than normal. In other words, at the phase range where the photosphere of a typical SN Ia is still in the silicon-rich layer, the photosphere of SN 2012fr may already have receded into the ${ }^{56} \mathrm{Ni}$-rich layer due to its extended distribution. Höflich et al. (2002) presented onedimensional, delayed-detonation models of a Chandrasekhar mass white dwarf that reproduce the luminosity-decline rate relation, from the sub-luminous to the most luminous SNe Ia, by varying the density at which the deflagration transitions to a detonation, $\rho_{\text {tr }}$, from values of $8-27 \times$ $10^{6} \mathrm{gm} \mathrm{cm}^{-3}$. The same model with a slightly higher transition density of $\rho_{\mathrm{tr}}=30 \times 10^{6} \mathrm{gm} \mathrm{cm}^{-3}$ produces a minimum velocity of $\mathrm{Si} / \mathrm{S}$ of $12,983 \mathrm{~km} \mathrm{~s}^{-1},{ }^{56} \mathrm{Ni}$ mass $=0.67 M_{\odot}$, and $M_{V}=-19.35 \mathrm{mag}$ (from private communication with Peter Höflich).

Maund et al. (2013), Childress et al. (2013), and Zhang et al. (2014) have pointed out that SN 2012fr shares certain properties with luminous events such as SN 1991T (Filippenko et al. 1992; Phillips et al. 1992; Ruiz-Lapuente et al. 1992), SN 1999aa ( $\mathrm{Li}$ et al. 2001; Garavini et al. 2004), and the previously mentioned SN 2005hj: slow-declining light curves, weak Si II $\lambda 6355$ absorption, and shallow photospheric velocity gradients. From this coincidence and the strong presence of HVFs during the premaximum phases, Zhang et al. (2014) proposed that SN 2012fr may represent a subset of the
1991T-like SNe Ia viewed at an angle where the ejecta has a clumpy or shell-like structure. However, SN 2012fr differs from 1991T-like events in three important ways, as follows: the high photospheric velocity of the Si II $\lambda 6355$ absorption at maximum light, the strong Si II absorption observed at early epochs, and the lack of strong features due to Fe III at maximum.

\subsection{A Distinct Subclass?}

Figure 18 illustrates the unusual nature of the Si II $\lambda 6355$ photospheric velocity evolution of SN $2012 \mathrm{fr}$ that began around 15 days before $t_{B_{\max }}$. The $1 \sigma$ dispersion about the average of the velocities for normal SNe Ia in the Wang et al. system (Folatelli et al. 2013) is shown for comparison, while the dashed lines correspond to a subset of the family of functions derived by Foley et al. (2011) to describe the velocity evolution of LVG and HVG SNe Ia. The extraordinarily shallow velocity evolution of SN 2012fr is unmatched, except for a handful of other HV events. The evolution of the Si II velocity for the two best observed of these-SN 2006is (Folatelli et al. 2013) and SN 2009ig (Marion et al. 2013)is shown for comparison in Figure 18. The positions of these two SNe in the branch diagram are also indicated in Figure 16.

SN 2009ig was extensively observed by Foley et al. (2012) and Marion et al. (2013). This slow-declining SN $\left(\Delta m_{15}(B)=0.89 \mathrm{mag}\right)$ was caught very early and it displayed strong HVF absorption in $\mathrm{Ca}$ II and $\mathrm{Si}$ II in the first spectra obtained $\sim 14$ days before $t_{B_{\max }}$. The earliest spectra were dominated by the HVFs. The first detection of photospheric 


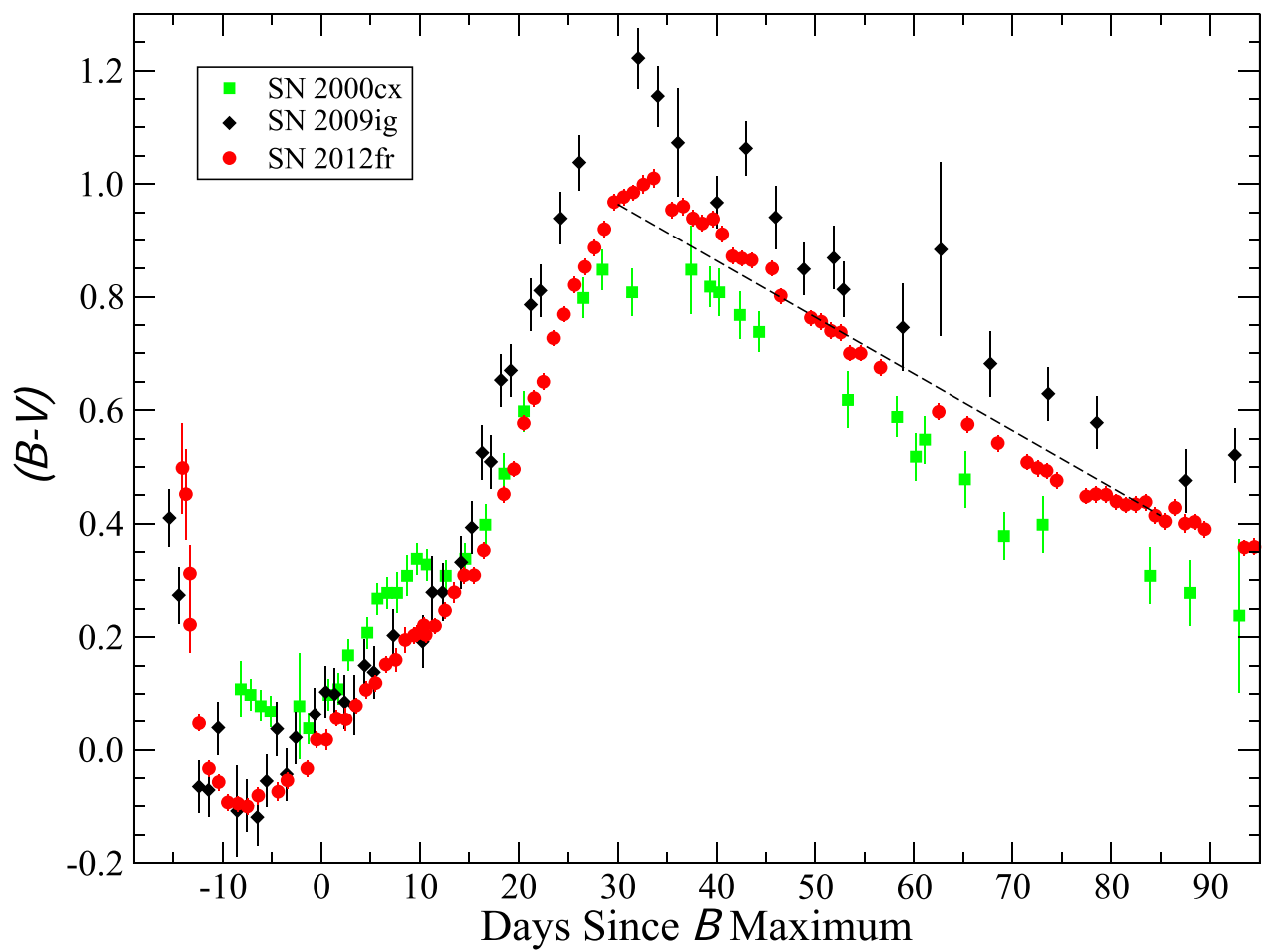

Figure 21. Comparison of the ( $B-V$ ) color evolution of SN 2000cx (green squares), SN 2009ig (black diamonds), and SN 2012fr (red circles). The abscissa is corrected for time dilation. The data for SN 2000cx are from Li et al. (2001), for SN 2009ig are from Foley et al. (2012), and for SN 2012fr are from this paper. No correction has been made for host-galaxy reddening for any of the SNes. The dashed line is the average Lira relation from Burns et al. (2014).

features began 12 days before $t_{B_{\max }}$. Both the HVF and photospheric absorption remained visible until 6 days before $t_{B_{\max }}$, when the HVF absorption was no longer detectable. The optical spectral evolution of the SN 2009ig was remarkably similar to that of SN 2012fr (Childress et al. 2013), with the only significant differences being: (1) the higher velocity, broader HVF absorption in the earliest (day -14) spectrum of SN 2012fr; (2) the longer persistence of the HVF Ca II and Si II absorption in SN 2012fr; and (3) the narrower Ca II, Fe II, S II, and Si II photospheric absorption in SN 2012fr from maximum onward (seen most clearly in the Ca II triplet). The photometric evolution of both SNe was also striking similar, with the most significant difference being in the $I$ band, where the secondary maximum of SN $2012 \mathrm{fr}$ occurs several days later than that of SN 2009ig. Like SN 2012fr, SN 2009ig was discovered $<1$ day after explosion (Foley et al. 2012). The $R$-band light curve of SN 2009ig is compared with the $\mathrm{gr}_{\mathrm{LSQ}}$ light curve of SN 2012fr in Figure 19, from which it is clear that the rise time to $t_{B_{\max }}$ of SN 2009ig was somewhat longer than that of SN 2012fr. The unfiltered observation corresponds to the discovery image of SN 2009ig. Unfortunately, the last nondetection was four days before discovery, making it difficult to determine with certainty that the early rise of SN 2009ig showed the same broken power-law morphology that was observed for SN 2012fr. However, we note that the shape of $R$-band light curve of SN 2009ig closely mimics that of the $\mathrm{gr}_{\mathrm{LSQ}}$ light curve of $\mathrm{SN} 2012 \mathrm{fr}$ for much of the rise to maximum, which suggests a similar morphology at the earliest epochs following explosion.

SN 2006is, has been discussed by Folatelli et al. (2013). Photometrically, it was also quite a slow decliner, with
$\Delta m_{15}(B)=0.80 \mathrm{mag}$ (Stritzinger et al. 2011). Unfortunately, spectroscopic observations did not begin until maximum, and so it is unknown whether this SN also displayed strong HVF absorption at early epochs. Nevertheless, as shown in Figure 14 of Folatelli et al. (2013), the maximum-light spectra of SNe 2006is and 2009ig were remarkably similar.

From this above discussion, we conclude that SNe 2006is, 2009ig, and 2012fr were similar events sharing the following characteristics:

1. All three were slow decliners $\left(\Delta m_{15}(B)=0.8-0.9\right.$ mag $)$.

2. All three occupied a similar region of the branch diagram near the edge of the $\mathrm{CN}$ and SS distributions (see Figure 16).

3. All three displayed unusually shallow Si II velocity gradients that are consistent with $\mathrm{LVG}$ events in the Benetti et al. (2005) classification scheme but at velocities $\gtrsim 12,000 \mathrm{~km} \mathrm{~s}^{-1}$, which place them in the Wang et al. (2009) HV class.

Moreover, SNe 2009ig and 2012fr displayed remarkably strong HVFs that persisted to maximum light. Unfortunately, SN 2006is was not discovered early enough to determine if it also shared this characteristic.

Very few other SNe Ia have displayed this particular combination of properties. The most notable exception is the peculiar SN 2000cx that was extensively observed by Li et al. (2001) and Candia et al. (2003). In addition to the high, nearly constant evolution of the Si II velocities (see Figure 18), SN 2000cx displayed remarkably strong Ca II HVFs at velocities of $>20,000 \mathrm{~km} \mathrm{~s}^{-1}$ that persisted to maximum light (Branch et al. 2004; Thomas et al. 2004). Interestingly, the evolution of the expansion velocities of the IMEs as deduced 
from the Si II $\lambda 6355$ and S II $\lambda \lambda 5449,5622$ lines (Li et al. 2001) closely resembles the evolution that was observed for SN 2012fr. Optical and NIR photometry of SN 2000cx also revealed certain anomalies in its photometric behavior, as follows: (1) an asymmetric $B$-band light curve, with a relatively fast rise from -10 days to maximum but then a slow postmaximum decline $\left(\Delta m_{15}(B)=0.93 \mathrm{mag}\right)$; (2) a weaker and earlier-occurring $I$-band secondary maximum than would be expected for such a slow decline rate; and $(3)$ a $(B-V)$ color evolution displaying several peculiarities, including a brief plateau phase of nearly constant color that began $\sim 1$ week after maximum and a strikingly bluer color than predicted by the Lira relation from $\sim 35$ to 90 days after maximum.

It is tempting to postulate that SNe 2006is, 2009ig, and 2012 fr were closely related to SN 2000cx and to its apparent twin, SN 2013bh (Silverman et al. 2013). The difference is that SN 2000cx was a fairly extreme SS event in the Branch et al. (2006, 2009) classification scheme (see Figure 16). Interestingly, SN 2012fr and, to a lesser extent, SN 2009ig also resembled SN 2000cx in displaying a $B$-band light curve that rose rather quickly to maximum but then declined more slowly than average after maximum. This is illustrated in Figure 20. Here, the normalized $B$ light curves are plotted with respect to $t_{B_{\max }}$, with time dilation taken into account. The Goldhaber et al. (2001) $B$ band Parab-18 template is also plotted, although it has been stretched to match the observed decline rates of each SN. The unusually rapid rise to maximum of SN 2000cx stands out clearly in this figure and is mimicked to a large extent by SN 2012fr. SN 2009ig also initially appeared to rise somewhat more quickly than the Goldhaber et al. template, although this difference would not have been so obvious if this event had not been discovered so early. Li et al. (2001) also called attention to a peculiarity in the $(B-V)$ evolution of SN 2000cx, which showed a phase of nearly constant color at $(B-V) \sim 0.3$ mag between 6 and 15 days past $t_{B_{\max }}$. As seen in Figure 21 , the $(B-V)$ evolution of SN $2012 \mathrm{fr}$ showed a similar, nearly constant color of $(B-V) \sim 0.2$ mag for a few days centered around day +10 . Unfortunately, the photometry of SN 2009ig is insufficiently precise to discern whether it also displayed such a peculiarity. However, Figure 21 shows that a change in the slope of the $(B-V)$ evolution of this $\mathrm{SN}$ occurred around day +10 , as it did for SN $2012 \mathrm{fr}$.

\section{Conclusions}

We have presented densely sampled, high quality, six-band optical photometry of the Type Ia SN 2012fr in the Fornax Cluster member NGC 1365. The data span epochs from 13 days before to 140 days after the epoch of $t_{B_{\max }}$, with typical errors below $2 \%$. We also present similarly high quality NIR and UV photometric data sets. Based on these observations, we conclude the following:

1. SN 2012fr was a slow declining $\left(\Delta m_{15}(B)=\right.$ $0.82 \pm 0.03 \mathrm{mag})$, luminous event. From the observed colors at maximum, the evolution of the $(B-V)$ color at later epochs, and the lack of detectable host-galaxy interstellar $\mathrm{Na}$ I D absorption in high-dispersion spectra, we estimate that SN 2012fr suffered little or no hostgalaxy reddening, adopting a conservative value of $E(B-V)_{\text {host }}=0.03 \pm 0.03 \mathrm{mag}$.

2. Analysis of the optical and NIR light curves shows that the luminosity of SN 2012fr was completely normal for its decline rate.
3. The images obtained by the LSQ survey tightly constrain the epoch of the explosion to $16.87 \pm 0.46$ days before $t_{B_{\max }}$ or $16.5 \pm 0.5$ days before the bolometric maximum. The luminosity of the SN increased nearly linearly at first, transitioning to a faster rising phase $\sim 2.5$ days after explosion. This behavior is well-fitted by an explosion model with moderate mixing of ${ }^{56} \mathrm{Ni}$ in the ejecta.

4. The densely sampled bolometric light curve derived from our UV, optical, and NIR photometry indicates that SN 2012fr peaked at a luminosity of $L_{\mathrm{Bol}}=1.35 \pm 0.14 \times$ $10^{43} \mathrm{erg} \mathrm{s}^{-1}$. By combining this value with the measured rise time, we estimate a ${ }^{56} \mathrm{Ni}$ mass of $0.60 \pm 0.15 M_{\odot}$ using Arnett's rule. This amount is consistent with an independent measurement of the ${ }^{56} \mathrm{Ni}$ mass from nebular spectra.

5. Despite its normal luminosity, SN 2012fr displayed spectroscopic properties that set it apart from most other SNe Ia. The classification as a Branch et al. (2006) SS/ $\mathrm{CN}$ event, with HV Si II absorption in the Wang et al. (2009) system but with a shallow velocity gradient (LVG using the Benetti et al. 2005 criteria) is uncommon and reminiscent of the peculiar SN 2000cx. Like SN 2000cx, SN 2012fr also displayed a fast rise to maximum and a much slower post-maximum decline. Finally, the velocity of the inner edge of the IMEs in SN 2012fr appears to have been $\sim 11,000 \mathrm{~km} \mathrm{~s}^{-1}$, or approximately double that of a typical SN Ia such as SN 2011fe. We call attention to two other SNe Ia, 2006is and 2009ig, that showed photometric and spectroscopic properties similar to those of SN 2012fr and we suggest that all three, along with SN 2000cx, may form a distinct subclass of SNe Ia.

6. The Cepheid-based distance to its host-galaxy is already available, which adds SN 2012fr to the still relatively small number of nearby $\mathrm{SNe}$ Ia that are suitable for measuring the value of the Hubble constant, $H_{\mathrm{o}}$.

We acknowledge Wei Kang Zheng for his help in providing discovery images of SN 2009ig and for his useful discussion. The work of the CSP has been supported by the National Science Foundation under grants AST0306969, AST0607438, AST1008343, AST1613426, AST1613455, and AST1613472. M.S. and C.C. acknowledge the generous support from the Danish Agency for Science and Technology and Innovation through a Sapere Aude Level 2 grant. E.Y.H. is supported by Florida Space Grant Consortium. M.S. is also supported by a research grant (13261) from VILLUM FONDEN.

\section{Appendix A \\ Throughput Functions and Zero Points for the LSQ, Tarot, Slooh, and BOSS Filters}

\section{A.1. La Silla-QUEST}

The relative throughput of the LSQ gr filter is plotted in panel (a) of Figure 22. This was constructed by multiplying the filter and CCD quantum efficiency curves given by Baltay et al. (2007, 2013) with a reflectivity curve for aluminum and an atmospheric transmission spectrum appropriate for the La Silla Observatory. The transmission of the corrector of the ESO Schmidt telescope was assumed to be flat over the spectral region covered by the filter. LSQ images were processed by the LSQ pipeline before being given to us. 


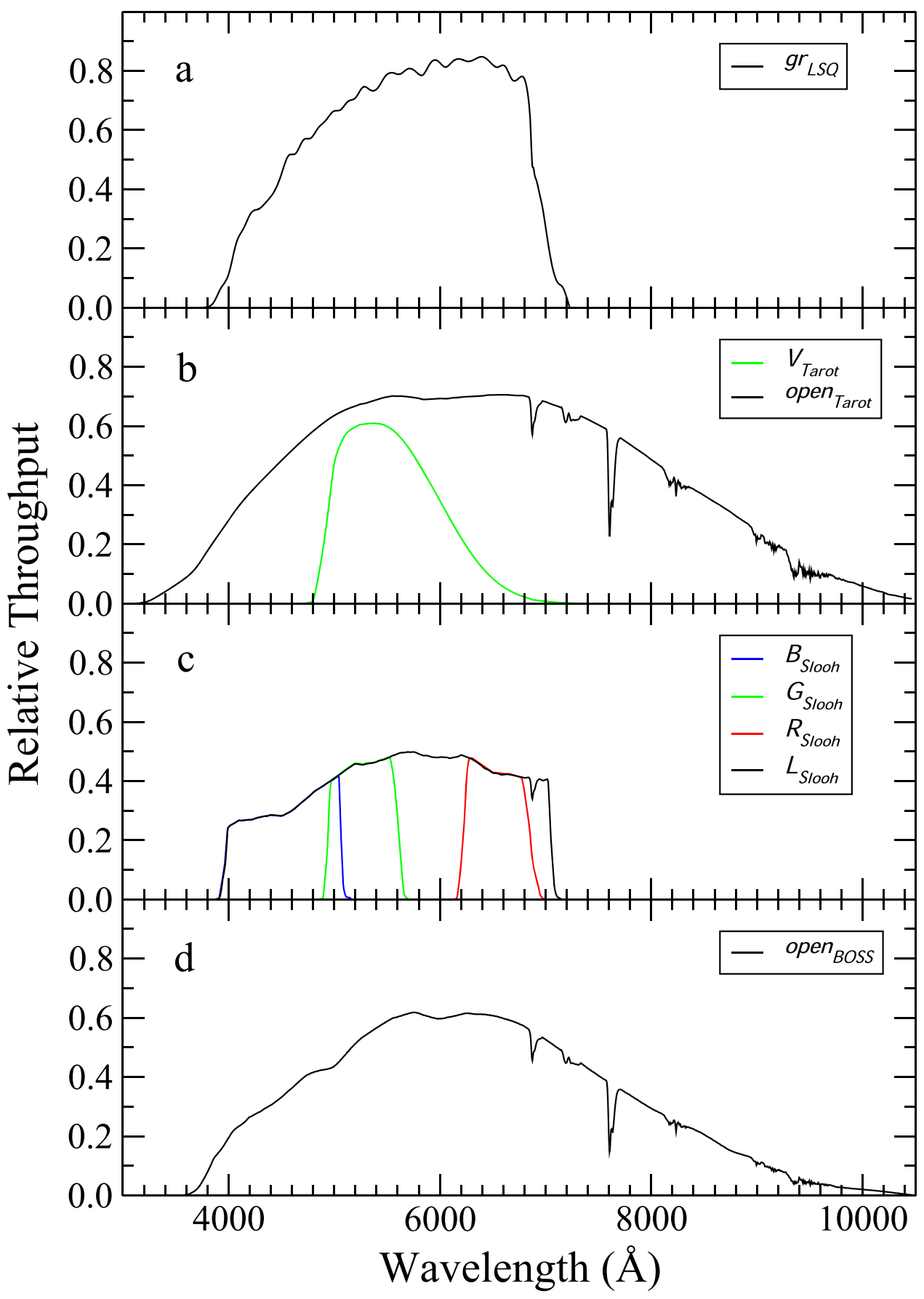

Figure 22. Relative throughput functions of the LSQ, Tarot, Slooh, and BOSS filters.

We measured the instrumental PSF photometry of SN 2012fr and field stars in the LSQ images using DAOPhot routines (Stetson 1987). The final magnitudes for the local sequence stars in the natural system of the LSQ gr filter were calculated using the following procedure:

1. Figure 23 shows color-color diagrams of $\left(V_{\mathrm{CSP}}-g r_{\mathrm{LSQ}}\right)$ and $\left(r_{\mathrm{CSP}}-g r_{\mathrm{LSQ}}\right)$ colors versus $(g-r)_{\mathrm{CSP}}$ derived from synthetic photometry of main-sequence stars, ${ }^{31}$ selected from the Pickles (1998) stellar library spectra. The zero

\footnotetext{
31 According to Finlator et al. (2000), 99\% of the field stars observed in the Sloan Digital Sky Survey are on the main sequence.
}

point of the synthetic photometry using the LSQ gr filter throughput function was set by requiring $\operatorname{gr}_{\mathrm{LSQ}}=0.0$ for Vega ( $\alpha$ Lyr). ${ }^{32}$ Likewise, synthetic magnitudes in the CSP $V, g$, and $r$ filters were calculated using the throughput functions and zero points given by Krisciunas et al. (2017).

2. The photometry of the local sequence stars in the field of SN 2012fr are plotted in Figure 23 as red circles. The zero point for the instrumental magnitudes of the local sequence stars was found by shifting the photometric

\footnotetext{
32 Vega spectrum from CALSPEC: ftp://ftp.stsci.edu/cdbs/calspec/ascii/ alpha_lyr_stis_005.ascii.
} 


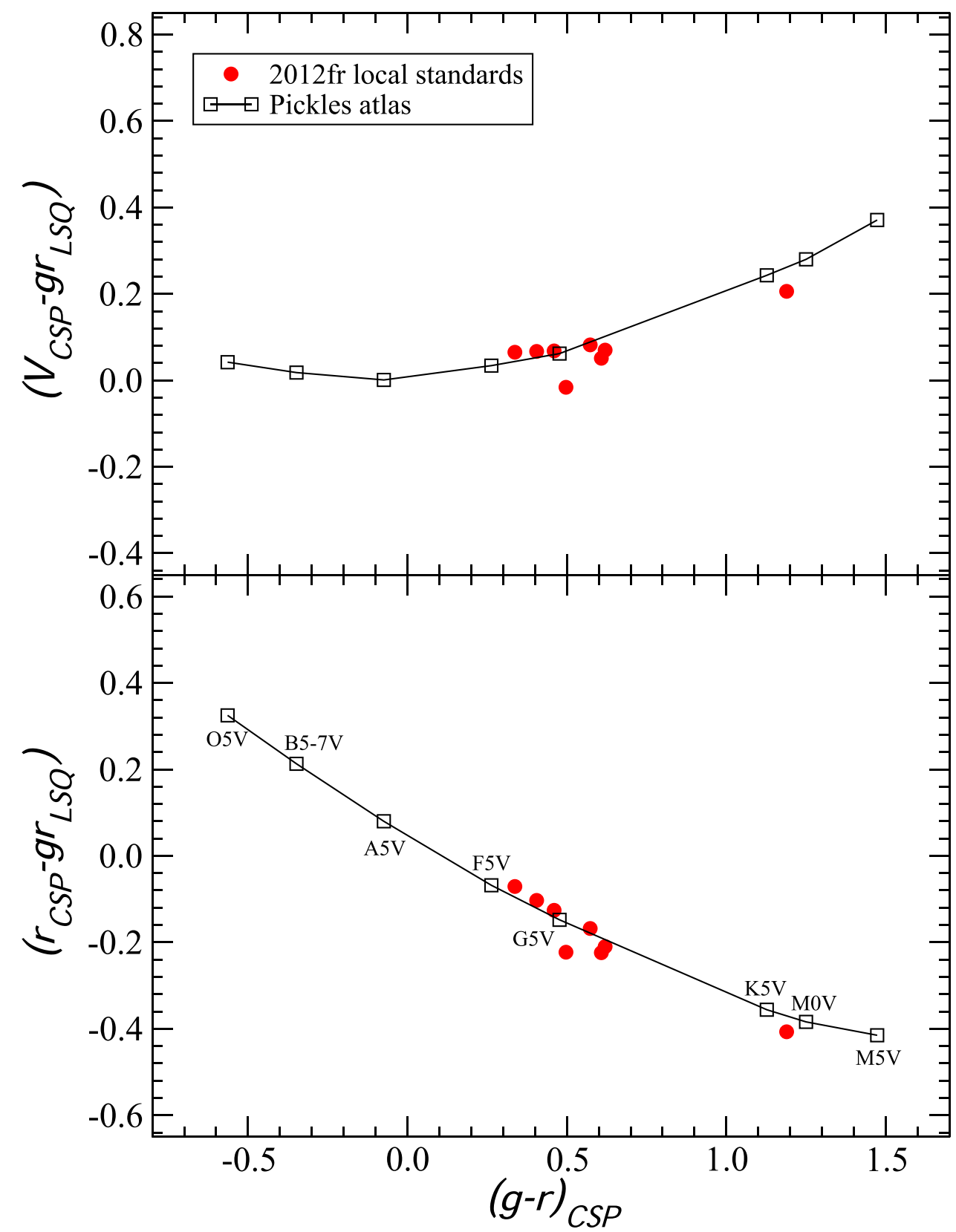

Figure 23. Color-color plots to convert CSP $V$ and $r$ magnitudes to the natural system magnitudes for the LSQ gr filter. The black curves show synthetic photometry carried out using the Pickles (1998) stellar atlas. The red points correspond to observations of the local sequence stars in the field of SN $2012 \mathrm{fr}$.

measurements along the $y$-axis to match the sequence defined by the Pickles stars.

The resulting magnitudes for the local sequence stars in the natural system of the LSQ gr filter are given in Table 9. Our measurement of the magnitude of SN 2012fr on 2012 October 26 UT in the natural system of the LSQ gr filter is given in Table 10.

SN 2012fr was not detected in a pair of $g_{L S Q}$ images acquired on 2012 October 25.34 UT (see Figure 4). We performed aperture photometry at the location of SN 2012fr on these images using the IRAF apphot package and we calibrated the results using the local sequence stars. This procedure implies 3- $\sigma$ limits of $\mathrm{gr}_{\mathrm{LSQ}}>20.38 \mathrm{mag}$ and $\mathrm{gr}_{\mathrm{LSQ}}>20.34$, respectively. This limit was verified by placing artificial sources with increasing magnitudes at the location of SN 2012fr in these images and then carrying out photometry on the resulting images. The artificial source was recovered when it was $m=20.34 \mathrm{mag}$, which is in excellent agreement with the previously given measured upper limits.

\section{A.2. TAROT}

The throughput of the TAROT open system was calculated from the detailed information given in Klotz et al. (2008). Panel (b) of Figure 22 shows the resulting sensitivity function, which includes atmospheric transmission typical of La Silla. Fully reduced TAROT images were provided to us by Alain Klotz.

We computed PSF photometry on the images with DAOPhot. Magnitudes for the local sequence stars in the natural system of the TAROT open filter were derived in the same manner as described previously for the LSQ gr filter. Figure 24 shows $\left(V_{\mathrm{CSP}}-\mathrm{open}_{\mathrm{TAROT}}\right)$ and $\left(r_{\mathrm{CSP}}-\mathrm{open}_{\mathrm{TAROT}}\right)$ colors for the 
Note. The values in parentheses are $1 \sigma$ measurement uncertainties and they are given in millimag.

Table 9

LSQ, TAROT, BOSS, and Slooh Natural System Photometry of SN 2012fr Local Sequence Stars

\begin{tabular}{|c|c|c|c|c|c|c|c|c|c|c|}
\hline Star ID & R.A. & Decl. & $\begin{array}{l}\mathrm{gr}_{\mathrm{LSQ}} \\
(\mathrm{mag})\end{array}$ & $\begin{array}{l}\text { open }_{\text {TAROT }} \\
\text { (mag) }\end{array}$ & $\begin{array}{c}V_{\text {TAROT }} \\
(\mathrm{mag})\end{array}$ & $\begin{array}{l}\text { open }_{\text {Boss }} \\
\text { (mag) }\end{array}$ & $\begin{array}{l}R_{\text {Slooh }} \\
\text { (mag) }\end{array}$ & $\begin{array}{l}G_{\text {Slooh }} \\
\text { (mag) }\end{array}$ & $\begin{array}{l}B_{\text {Slooh }} \\
(\mathrm{mag})\end{array}$ & $\begin{array}{l}L_{\text {Slooh }} \\
\text { (mag) }\end{array}$ \\
\hline 1 & 03:33:55.48 & $-36: 07: 19.0$ & $\ldots$ & $12.748(007)$ & 13.301(002) & $12.844(034)$ & $12.484(014)$ & $14.403(028)$ & 13.591(016) & $13.242(006)$ \\
\hline 2 & 03:33:33.67 & $-36: 07: 14.1$ & $\ldots$ & $13.293(007)$ & $\ldots$ & 13.323(014) & $13.134(023)$ & $14.127(023)$ & $13.659(020)$ & $13.511(008)$ \\
\hline 3 & $03: 33: 49.55$ & $-36: 07: 42.8$ & 14.513(005) & $14.376(011)$ & $14.541(003)$ & $14.386(026)$ & $14.130(040)$ & $14.975(042)$ & $14.539(033)$ & $14.534(010)$ \\
\hline 4 & 03:33:55.66 & $-36: 08: 38.2$ & 14.994(005) & $14.824(015)$ & $15.006(005)$ & $14.789(035)$ & $14.399(048)$ & $15.721(077)$ & $15.222(074)$ & $15.001(015)$ \\
\hline 5 & 03:33:40.67 & $-36: 10: 52.9$ & 15.041(006) & $14.822(015)$ & $15.004(015)$ & $14.863(025)$ & $14.644(052)$ & $15.507(060)$ & $15.131(052)$ & $15.074(017)$ \\
\hline 6 & 03:33:57.36 & $-36: 09: 24.0$ & $16.654(012)$ & $16.505(053)$ & $16.608(028)$ & $16.421(067)$ & $16.620(288)$ & $17.028(228)$ & $16.909(365)$ & $16.600(052)$ \\
\hline 7 & 03:33:54.96 & $-36: 09: 09.6$ & 16.641(011) & 16.501(058) & $16.652(031)$ & $16.407(059)$ & $15.988(163)$ & $17.751(516)$ & $16.883(342)$ & $16.685(054)$ \\
\hline 8 & $03: 33: 46.83$ & $-36: 03: 04.1$ & $\ldots$ & $\ldots$ & $\ldots$ & $13.848(018)$ & $13.676(026)$ & $14.380(137)$ & $14.051(027)$ & $\ldots$ \\
\hline 9 & 03:33:30.90 & $-36: 02: 08.1$ & $\ldots$ & $15.169(019)$ & $15.805(008)$ & $15.329(039)$ & $14.731(050)$ & $16.886(202)$ & $16.311(179)$ & $15.697(024)$ \\
\hline 10 & 03:33:39.87 & $-36: 01: 27.2$ & $\ldots$ & $16.244(042)$ & $16.564(015)$ & $16.331(041)$ & $15.903(179)$ & $17.800(565)$ & $16.677(278)$ & $16.422(045)$ \\
\hline 11 & 03:33:10.58 & $-36: 03: 41.9$ & $\ldots$ & $\cdots$ & $\ldots$ & 13.334(064) & $13.072(031)$ & $13.806(103)$ & $13.487(017)$ & \\
\hline 12 & 03:33:16.73 & $-36: 05: 08.9$ & $15.198(005)$ & 14.651(014) & $15.238(005)$ & $14.788(045)$ & $14.274(051)$ & 16.331(117) & $15.437(078)$ & $15.144(016)$ \\
\hline 13 & $03: 33: 15.45$ & $-36: 03: 47.4$ & $16.640(011)$ & $16.428(048)$ & 16.641(016) & $16.477(095)$ & $15.831(139)$ & $17.407(319)$ & $16.374(183)$ & $16.524(054)$ \\
\hline 14 & 03:33:58.35 & $-36: 02: 18.5$ & $\ldots$ & $16.447(056)$ & $16.518(014)$ & 16.401(067) & $15.854(138)$ & $16.936(242)$ & $16.509(195)$ & $16.478(049)$ \\
\hline 15 & 03:34:01.65 & $-36: 08: 45.9$ & $\ldots$ & $15.095(015)$ & $15.306(005)$ & $15.100(046)$ & $15.012(098)$ & $15.741(073)$ & $15.404(075)$ & $15.286(017)$ \\
\hline 16 & 03:33:28.37 & $-36: 14: 38.0$ & $\cdots$ & $15.573(022)$ & $15.980(010)$ & $15.670(037)$ & $15.280(084)$ & $16.740(166)$ & $16.030(132)$ & $\cdots$ \\
\hline 17 & 03:33:15.26 & $-36: 14: 39.3$ & $\cdots$ & $\cdots$ & $\cdots$ & $15.313(070)$ & $14.890(052)$ & $16.319(113)$ & $15.967(124)$ & $\cdots$ \\
\hline 18 & 03:33:02.16 & $-36: 13: 28.2$ & $\cdots$ & $14.588(013)$ & 14.892(004) & 14.661(110) & $14.498(047)$ & $15.590(055)$ & $15.172(062)$ & $14.929(012)$ \\
\hline 19 & $03: 33: 12.50$ & $-36: 11: 10.3$ & 16.137(010) & $15.857(031)$ & $16.077(010)$ & $15.934(068)$ & $15.662(112)$ & $16.641(152)$ & $16.563(222)$ & $16.080(033)$ \\
\hline 20 & 03:33:52.46 & $-36: 14: 47.6$ & $\cdots$ & $\cdots$ & $\cdots$ & $15.993(046)$ & $15.674(114)$ & $16.374(120)$ & $16.358(188)$ & $\cdots$ \\
\hline 21 & 03:33:04.96 & $-36: 02: 30.6$ & $\cdots$ & $16.184(039)$ & $16.898(022)$ & $16.367(133)$ & $15.798(141)$ & $18.654(331)$ & $17.229(430)$ & 16.801(066) \\
\hline 22 & 03:33:14.32 & $-36: 12: 13.6$ & $\cdots$ & $16.394(048)$ & $17.017(033)$ & $16.505(052)$ & $15.892(174)$ & $18.372(820)$ & $17.872(669)$ & $16.976(073)$ \\
\hline 23 & 03:33:12.02 & $-36: 11: 49.2$ & $\ldots$ & $15.905(035)$ & $16.774(018)$ & $16.128(056)$ & $15.612(098)$ & $17.460(446)$ & $16.777(238)$ & $16.623(051)$ \\
\hline
\end{tabular}


Table 10

LSQ, TAROT, BOSS, and Slooh Natural System Photometry of SN 2012fr

\begin{tabular}{|c|c|c|c|c|c|c|c|c|c|}
\hline $\begin{array}{l}\text { JD } \\
\text { (days) }\end{array}$ & $\begin{array}{l}\text { Phase } \\
\text { (days) }\end{array}$ & $\begin{array}{l}\mathrm{gr}_{\mathrm{LSQ}} \\
(\mathrm{mag})\end{array}$ & $\begin{array}{l}\text { open }_{\text {TAROT }} \\
\text { (mag) }\end{array}$ & $\begin{array}{l}V_{\text {TAROT }} \\
\text { (mag) }\end{array}$ & $\begin{array}{l}\text { open }_{\text {Boss }} \\
\text { (mag) }\end{array}$ & $\begin{array}{l}R_{\text {Slooh }} \\
\text { (mag) }\end{array}$ & $\begin{array}{l}G_{\text {Slooh }} \\
\text { (mag) }\end{array}$ & $\begin{array}{l}B_{\text {Slooh }} \\
(\mathrm{mag})\end{array}$ & $\begin{array}{l}L_{\text {Slooh }} \\
(\mathrm{mag})\end{array}$ \\
\hline 2456226.69 & -16.41 & $17.705(029)$ & $\ldots$ & $\ldots$ & $\ldots$ & $\ldots$ & $\ldots$ & $\ldots$ & $\ldots$ \\
\hline 2456226.77 & -16.33 & $17.364(021)$ & $\cdots$ & $\cdots$ & $\cdots$ & $\cdots$ & $\cdots$ & $\cdots$ & $\cdots$ \\
\hline 2456227.55 & -15.55 & $\cdots$ & $15.940(061)$ & $\cdots$ & $\cdots$ & $\cdots$ & $\cdots$ & $\cdots$ & $\cdots$ \\
\hline 2456227.62 & -15.48 & $\ldots$ & $\ldots$ & $\ldots$ & $\ldots$ & $15.663(139)$ & $16.112(149)$ & $16.426(274)$ & $16.238(0.064)$ \\
\hline 2456228.86 & -14.24 & $\cdots$ & $\cdots$ & $\cdots$ & $15.214(029)$ & $\cdots$ & $\cdots$ & $\cdots$ & $\cdots$ \\
\hline 2456229.63 & -13.47 & $\cdots$ & $\cdots$ & $14.667(027)$ & $\cdots$ & $\cdots$ & $\cdots$ & $\cdots$ & $\cdots$ \\
\hline 2456229.67 & -13.43 & $\cdots$ & $\cdots$ & $14.573(021)$ & $\cdots$ & $\cdots$ & $\cdots$ & $\cdots$ & $\cdots$ \\
\hline 2456229.72 & -13.38 & $\cdots$ & $\cdots$ & $14.576(018)$ & $\cdots$ & $\cdots$ & $\cdots$ & $\cdots$ & $\cdots$ \\
\hline 2456229.76 & -13.34 & $\cdots$ & $\cdots$ & $14.520(020)$ & $\cdots$ & $\cdots$ & $\cdots$ & $\cdots$ & $\cdots$ \\
\hline 2456229.80 & -13.30 & $\cdots$ & $\cdots$ & $14.541(022)$ & $\cdots$ & $\cdots$ & $\cdots$ & $\cdots$ & $\cdots$ \\
\hline 2456229.91 & -13.20 & $\cdots$ & $\cdots$ & $\cdots$ & $14.408(011)$ & $\cdots$ & $\cdots$ & $\cdots$ & $\cdots$ \\
\hline 2456230.63 & -12.47 & $\cdots$ & $\cdots$ & $14.059(014)$ & $\cdots$ & $\cdots$ & $\cdots$ & $\cdots$ & $\cdots$ \\
\hline 2456230.67 & -12.43 & $\cdots$ & $\cdots$ & $14.004(015)$ & $\cdots$ & $\cdots$ & $\cdots$ & $\cdots$ & $\cdots$ \\
\hline 2456230.72 & -12.38 & $\cdots$ & $\cdots$ & $13.953(012)$ & $\cdots$ & $\cdots$ & $\cdots$ & $\cdots$ & $\cdots$ \\
\hline 2456230.76 & -12.34 & $\cdots$ & $\cdots$ & $13.976(026)$ & $\cdots$ & $\cdots$ & $\cdots$ & $\cdots$ & $\cdots$ \\
\hline 2456230.80 & -12.30 & $\cdots$ & $\cdots$ & 13.937(012) & $\cdots$ & $\cdots$ & $\cdots$ & $\cdots$ & $\cdots$ \\
\hline 2456230.94 & -12.16 & $\cdots$ & $\cdots$ & $\cdots$ & $13.791(015)$ & $\cdots$ & $\cdots$ & $\cdots$ & $\cdots$ \\
\hline 2456231.80 & -11.30 & $\cdots$ & $\cdots$ & $13.513(008)$ & $\cdots$ & $\cdots$ & $\cdots$ & $\cdots$ & $\cdots$ \\
\hline 2456232.63 & -10.47 & $\cdots$ & $\cdots$ & $13.238(005)$ & $\cdots$ & $\cdots$ & $\cdots$ & $\cdots$ & $\cdots$ \\
\hline 2456232.67 & -10.43 & $\cdots$ & $\cdots$ & $13.188(005)$ & $\cdots$ & $\cdots$ & $\cdots$ & $\cdots$ & $\cdots$ \\
\hline 2456232.72 & -10.38 & $\cdots$ & $\cdots$ & $13.175(006)$ & $\cdots$ & $\cdots$ & $\cdots$ & $\cdots$ & $\cdots$ \\
\hline 2456232.76 & -10.34 & $\cdots$ & $\cdots$ & $13.162(005)$ & $\cdots$ & $\cdots$ & $\cdots$ & $\cdots$ & $\cdots$ \\
\hline 2456234.63 & -8.47 & $\cdots$ & $\cdots$ & $12.733(003)$ & $\cdots$ & $\cdots$ & $\cdots$ & $\cdots$ & $\cdots$ \\
\hline 2456234.67 & -8.43 & $\cdots$ & $\cdots$ & $12.705(003)$ & $\cdots$ & $\cdots$ & $\cdots$ & $\cdots$ & $\cdots$ \\
\hline 2456234.72 & -8.40 & $\cdots$ & $\cdots$ & $12.688(003)$ & $\cdots$ & $\cdots$ & $\cdots$ & $\cdots$ & $\cdots$ \\
\hline 2456234.76 & -8.34 & $\cdots$ & $\cdots$ & $12.684(003)$ & $\cdots$ & $\cdots$ & $\cdots$ & $\cdots$ & $\cdots$ \\
\hline 2456234.80 & -8.30 & $\cdots$ & $\cdots$ & $12.688(003)$ & $\cdots$ & $\cdots$ & $\cdots$ & $\cdots$ & $\cdots$ \\
\hline
\end{tabular}

Note. The phase was computed relative to $t_{B_{\max }}=$ JD 2456243.1. The values in parentheses are $1 \sigma$ measurement uncertainties and they are given in millimag.

Pickles main-sequence stars plotted versus $(g-r)_{\mathrm{CSP}}$, with the zero point for the open 0.0 for Vega. The photometry of the local sequence stars in the field of SN 2012fr is plotted as red points after adjusting the zero points of the open TAROT $_{\text {instrumental magnitudes to }}$ provide the best fit to the Pickles stars. The final magnitudes for the local sequence stars in the natural system of the open $_{\text {TAROT }}$ filter are foundin Table 9, and the photometry of the $\mathrm{SN}$ is given in Table 10.

\section{A.3. Slooh}

Panel (c) of Figure 22 shows the throughput functions calculated for the Slooh $L R G B$ filters using information provided by E. Conseil (private communication). We include the atmospheric transmission appropriate for the Teide Observatory. The processed Slooh images were provided by E. Conseil. These displayed a strong gradient in the sky background that was subtracted prior to computing instrumental PSF magnitudes.

Although SN 2012fr is clearly detected in the Slooh images, it has asymmetric, low signal-to-noise ratio PSFs that are unsuitable for measuring magnitudes with our standard photometry tools. Therefore, to analyze these data, it was necessary to develop a specialized tool that produces a threedimensional model of the PSF using an isolated bright star in the images. The model is background subtracted and subsampled, and it is then fitted to the $\mathrm{SN}$ and local sequence stars in the image using an MCMC procedure where the amplitude and the center coordinates are fitted simultaneously.
An example of the PSF signal subtraction for the $B$-band image of the supernova and one of the local standards is shown in Figure 25.

The $B_{\text {Slooh }}$ and $G_{\text {Slooh }}$ filters are moderately well-matched to the CSP $B$ and $V$ filters, as shown in Figures 26(a) and (b). Here, $\left(B_{\text {Slooh }}-B_{\mathrm{CSP}}\right)$ and $\left(G_{\text {Slooh }}-V_{\mathrm{CSP}}\right)$ are plotted versus $(B-V)_{\mathrm{CSP}}$ for the Pickles stars with the zero points of the $B_{\text {Slooh }}$ and $G_{\text {Slooh }}$ magnitudes set, assuming $B_{\text {Slooh }}=$ $V_{\text {Slooh }}=0.0$ for Vega. The observed colors for the sequence stars, plotted as red points, have been adjusted to fit the synthetic colors by varying the zero points of the instrumental magnitudes. The final magnitudes of the local sequence stars in the natural systems of the $B_{\text {Slooh }}$ and $G_{\text {Slooh }}$ filters derived from this procedure are given in Table 9. The photometry of the SN in these filters is listed in Table 10 .

We also attempted to match the $R_{\text {Slooh }}$ filter to the $r_{\mathrm{CSP}}$ bandpass. The result is shown in the color-color diagram plotted in Figure $26(\mathrm{c})$, where $\left(R_{\text {Slooh }}-r_{\mathrm{CSP}}\right)$ is plotted versus $(g-r)_{\mathrm{CSP}}$ for the Pickles stars and assuming $R_{\text {Slooh }}=0.0$ for Vega. The observed trend that was measured from the photometry of the local sequence stars, as plotted by the red points, is seen to be consistent with the expectation from the synthetic photometry of the Pickles stars after adjusting for the zero point difference. The magnitudes of the local sequence stars in the natural system of the Slooh $R$ filter are given in Table 9, and photometry of the $\mathrm{SN}$ in this filter is given in Table 10. 


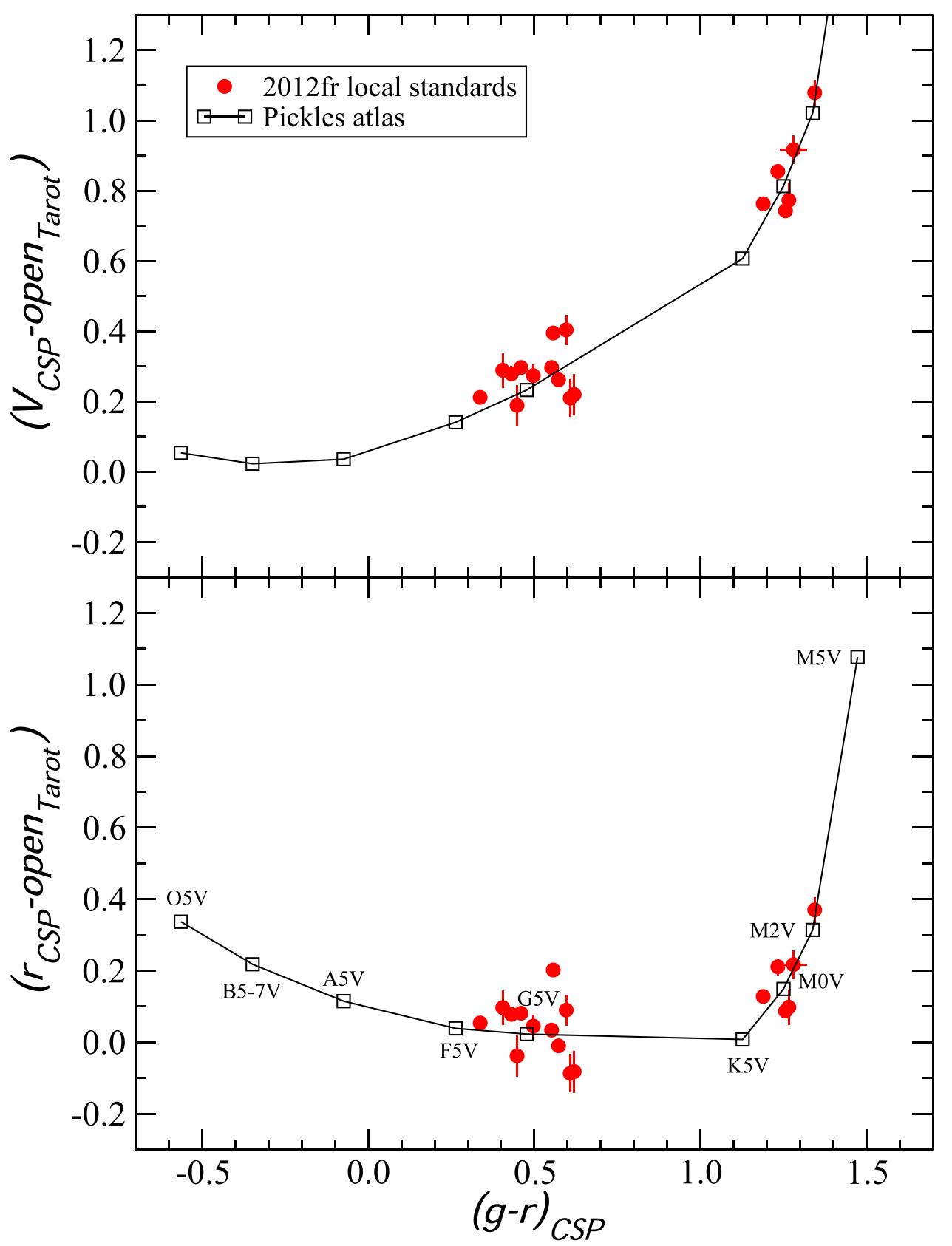

Figure 24. Color-color plots for converting CSP $V$ and $r$ magnitudes to the natural system magnitudes for the TAROT open filter. The black curves show synthetic photometry carried out using the Pickles (1998) stellar atlas. The red points correspond to observations of the local sequence stars in the field of SN $2012 \mathrm{fr}$.

The $L_{\text {Slooh }}$ (luminance) filter throughput function closely resembles that of the LSQ gr filter. This is confirmed in the color-color diagram shown in Figure 26(d). Here, $\left(L_{\text {Slooh }}-g r_{\text {LSQ }}\right)$ is plotted versus $(g-r)_{\mathrm{CSP}}$ from synthetic photometry of the Pickles main-sequence stars, where the zero point for the $L_{\text {Slooh }}$ filter has been calculated assuming $L_{\text {Slooh }}=0.0$ for Vega. The slope is nearly flat over the whole color range of the Pickles stars, which is consistent with a color term that is essentially zero. The measured colors of the local sequence stars are plotted as red points after adjusting the zero point of the $L_{\text {Slooh }}$ instrumental magnitudes to provide the best fit to the Pickles stars. Therefore, we assume that the $L_{\text {Slooh }}$ filter is in the same natural system as the $\operatorname{gr}_{\mathrm{LSQ}}$ filter. The photometry of the $\mathrm{SN}$ in the $L_{\text {Slooh }}$ filter is given in Table 10.

\section{A.4. BOSS}

The throughput of the BOSS open system was constructed from information provided by Stuart Parker, who observed SN 2012fr using a Celestron C14 $f / 10$ telescope with a $f / 6.3$ focal reducer attached before a Kodak KAF-3200ME CCD with coverglass. The transmission curves for the StarBright coatings on the telescope optics were found on the Celestron website, but they only covered the wavelength range of 4000-7500 A. In response to our inquiry, Celestron advised us that the "transmission falloff in the IR and UV bands was pretty severe" (private communication). The transmission of the CCD coverglass is given in a specification sheet that was supplied by the manufacturer, but they only covered the wavelength range from 3500 to $8500 \AA$. No information on the transmission of the focal reducer could be found. Hence, constructing the 

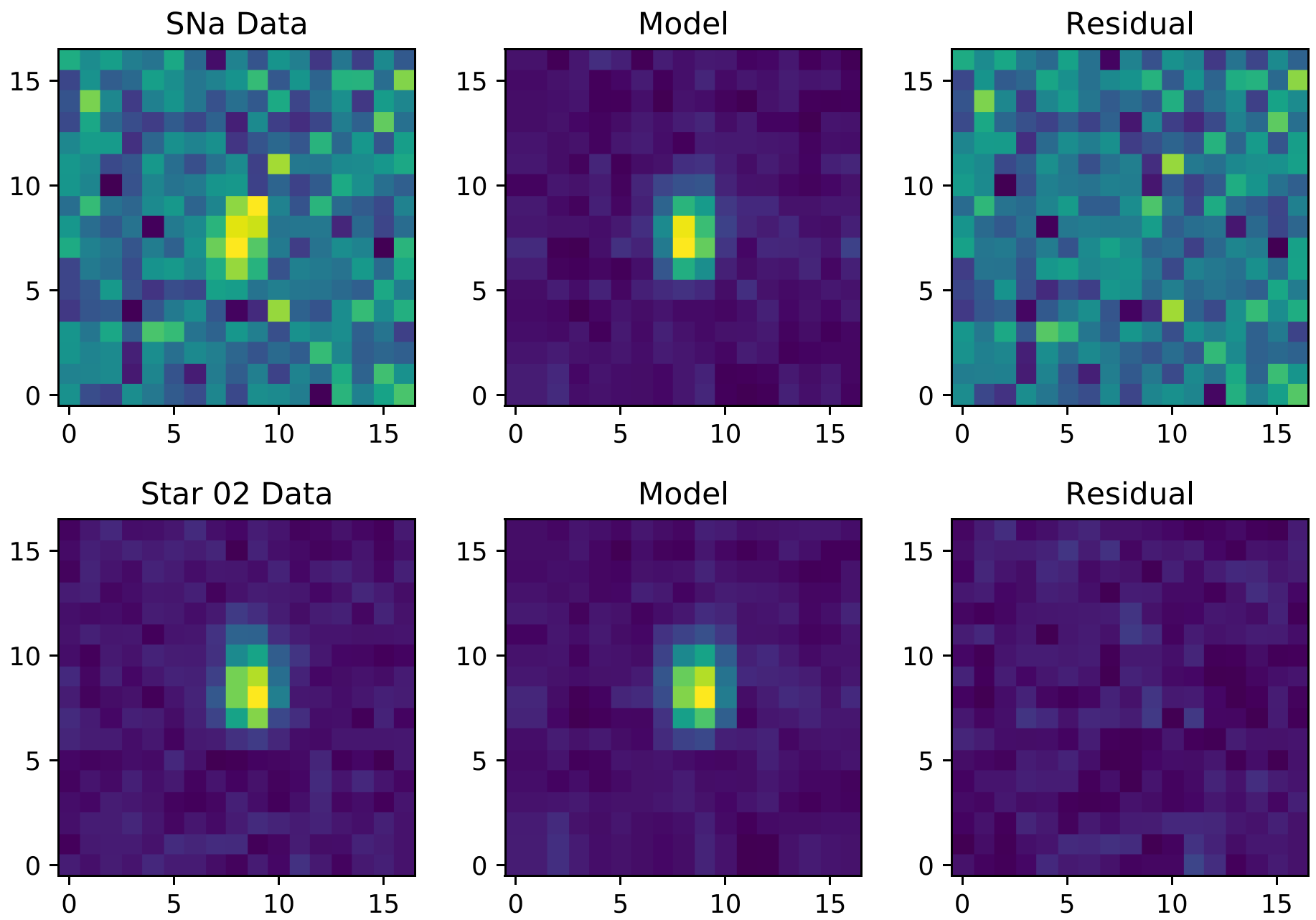

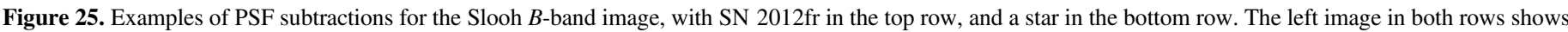
the original data, the center image shows the scaled PSF model, and the right image shows the subtractions of the scaled PSF model from the original data.

BOSS open filter throughput required some guesswork. Panel (d) of Figure 22 shows our final approximation, including the transmission of the atmosphere.

The BOSS images were processed by Stuart Parker using CCDsoft routines. PSF photometry was then carried out with DAOPhot. Final magnitudes for the local sequence stars in the natural system of the BOSS open filter were derived in the same manner as described previously for the LSQ gr filter. Figure 27 shows the $\left(V-\right.$ open $\left._{\text {BOSS }}\right)$ and $\left(r-\right.$ open $\left._{\text {BOSS }}\right)$ colors for the Pickles main-sequence stars plotted versus $(g-r)_{\mathrm{CSP}}$, with the zero point for the synthetic magnitudes chosen to give open $_{\mathrm{BOSS}}=0.0$ for Vega. The local sequence stars in the field of SN 2012fr are plotted as solid red circles in Figure 27 after

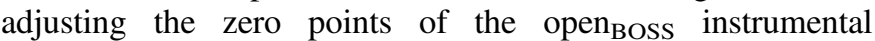
magnitudes to provide the best fit to the Pickles stars. Because of the uncertainties involved in constructing the throughput curve of the BOSS open system, these observations of the SN 2012fr local sequence are augmented by photometry (plotted with open red circles) of stars in the field of SN 2014do and a relatively low galactic latitude transient that was observed by both Parker and the CSP-II. In general, the observations are well-matched by the colors derived from the synthetic photometry. This provides confidence that the
Table 11

Milky Way and Host-galaxy Extinction Corrections and AB Magnitude Offsets

\begin{tabular}{lcccc}
\hline \hline Filter & $\begin{array}{c}\text { Effective } \\
\text { Wavelength }(\AA)\end{array}$ & $\begin{array}{c}\text { Milky Way } \\
A_{\lambda}(\mathrm{mag})\end{array}$ & $\begin{array}{c}\text { Host-galaxy } \\
A_{\lambda}(\mathrm{mag})\end{array}$ & $\begin{array}{c}\text { AB Magnitude } \\
\text { Offset }\end{array}$ \\
\hline$u v m 2$ & 2360 & 0.140 & 0.266 & +1.69 \\
$u$ & 3550 & 0.087 & 0.147 & -0.06 \\
$B$ & 4300 & 0.074 & 0.126 & -0.13 \\
$g$ & 4700 & 0.068 & 0.115 & -0.02 \\
$V$ & 5400 & 0.056 & 0.095 & -0.02 \\
$r$ & 6200 & 0.047 & 0.080 & -0.01 \\
$i$ & 7500 & 0.035 & 0.064 & +0.00 \\
$Y$ & 10000 & 0.020 & 0.038 & +0.64 \\
$J$ & 12500 & 0.015 & 0.026 & +0.90 \\
$H$ & 16600 & 0.009 & 0.017 & +1.34 \\
\hline
\end{tabular}

Note. For the $u v m 2$-filter, the $\mathrm{AB}$ shift was taken from https://swift.gsfc.nasa. gov/analysis/uvot_digest/zeropts.html. For the CSP-I $u$ BgVriYJH filters, the AB offsets are given in Krisciunas et al. (2017) in Table 16.

throughput function shown in panel (d) of Figure 22 is a reasonable representation of the BOSS open filter. The final magnitudes for the local sequence stars in the natural system of the BOSS open filter are given in Table 9 and the photometry 

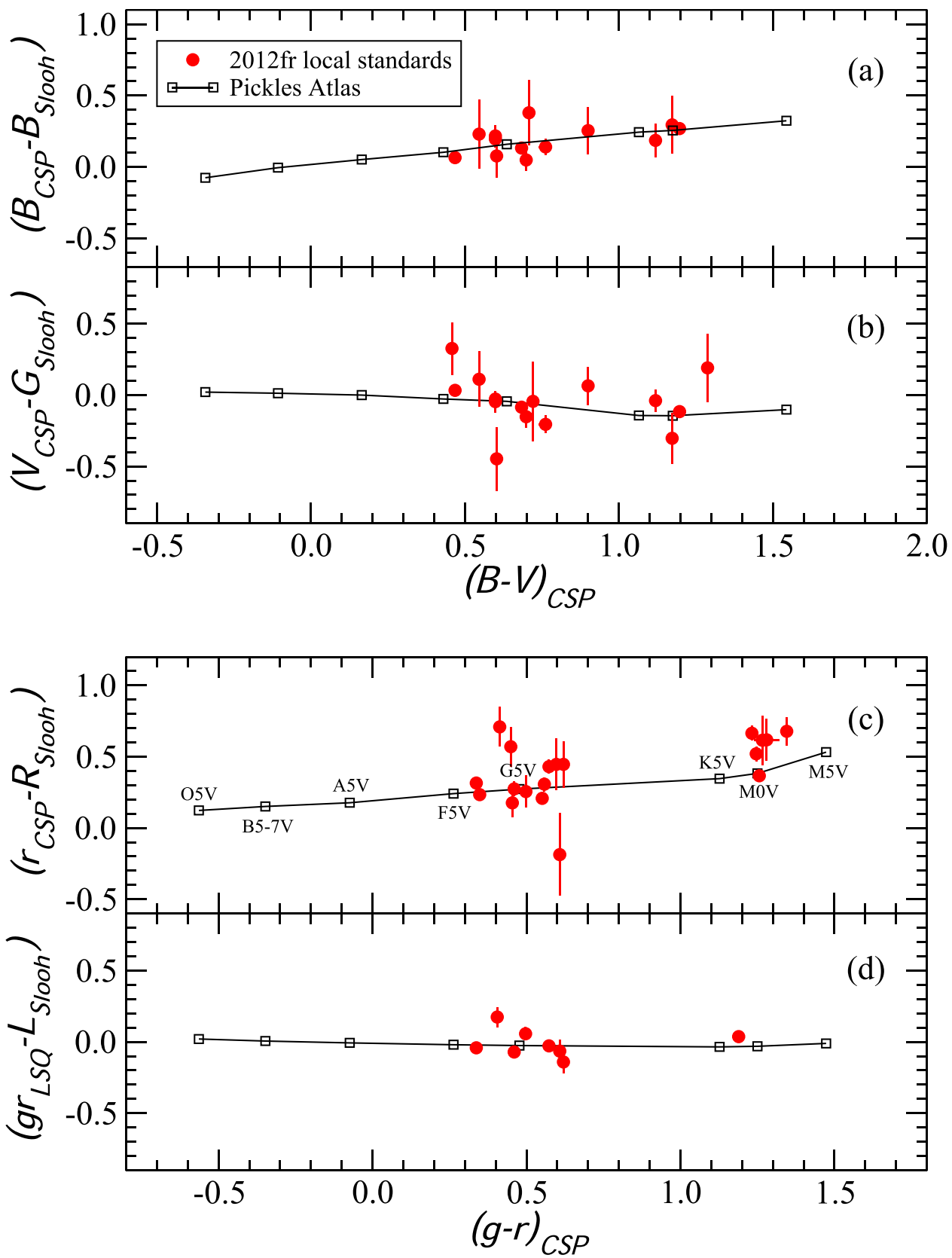

Figure 26. Color-color plots for converting (a) $B_{\mathrm{CSP}}$ magnitudes to natural system magnitudes in the $B_{\text {Slooh }}$ filter, (b) $V_{\mathrm{CSP}}$ magnitudes to natural system magnitudes in the $G_{\text {Sloo }}$ filter, (c) $r_{\mathrm{CSP}}$ magnitudes to natural system magnitudes in the $R_{\text {Slooh }}$ filter, and (d) $\mathrm{gr}_{\mathrm{LSQ}}$ magnitudes to natural system magnitudes in the $L_{\mathrm{Slooh}}$ filter. The black curves show synthetic photometry carried out using the Pickles (1998) stellar atlas. The red points correspond to observations of the local sequence stars in the field of SN 2012fr.

of the $\mathrm{SN}$ in the natural system of the BOSS open filter is given in Table 10.

\section{Appendix B \\ Bolometric Light Curve Construction}

As discussed in detail by Brown et al. (2016), producing a bolometric light curve from photometry of an object such as a SN Ia with a spectral energy distribution (SED) that differs significantly from those of stars is, at best, an approximate procedure. Consequently, we employed two different methods, which are detailed in this appendix.

\section{B.1. Photometric Trapezoidal Integration}

This method estimates the flux density from the observed photometry by simple trapezoidal integration of the fluxes derived from each filter. In addition, estimates must be made for the UV $\left(\lambda<\lambda_{u}\right)$ and infrared $\left(\lambda>\lambda_{H}\right)$ contributions-the former from the Swift $u v m 2$ photometry, and the latter from the Rayleigh-Jeans law. The following steps are involved:

1. The NIR $Y J H$ magnitudes are fitted to match optical phase coverage.

2. The optical magnitudes are interpolated for nights where observations were not available. 


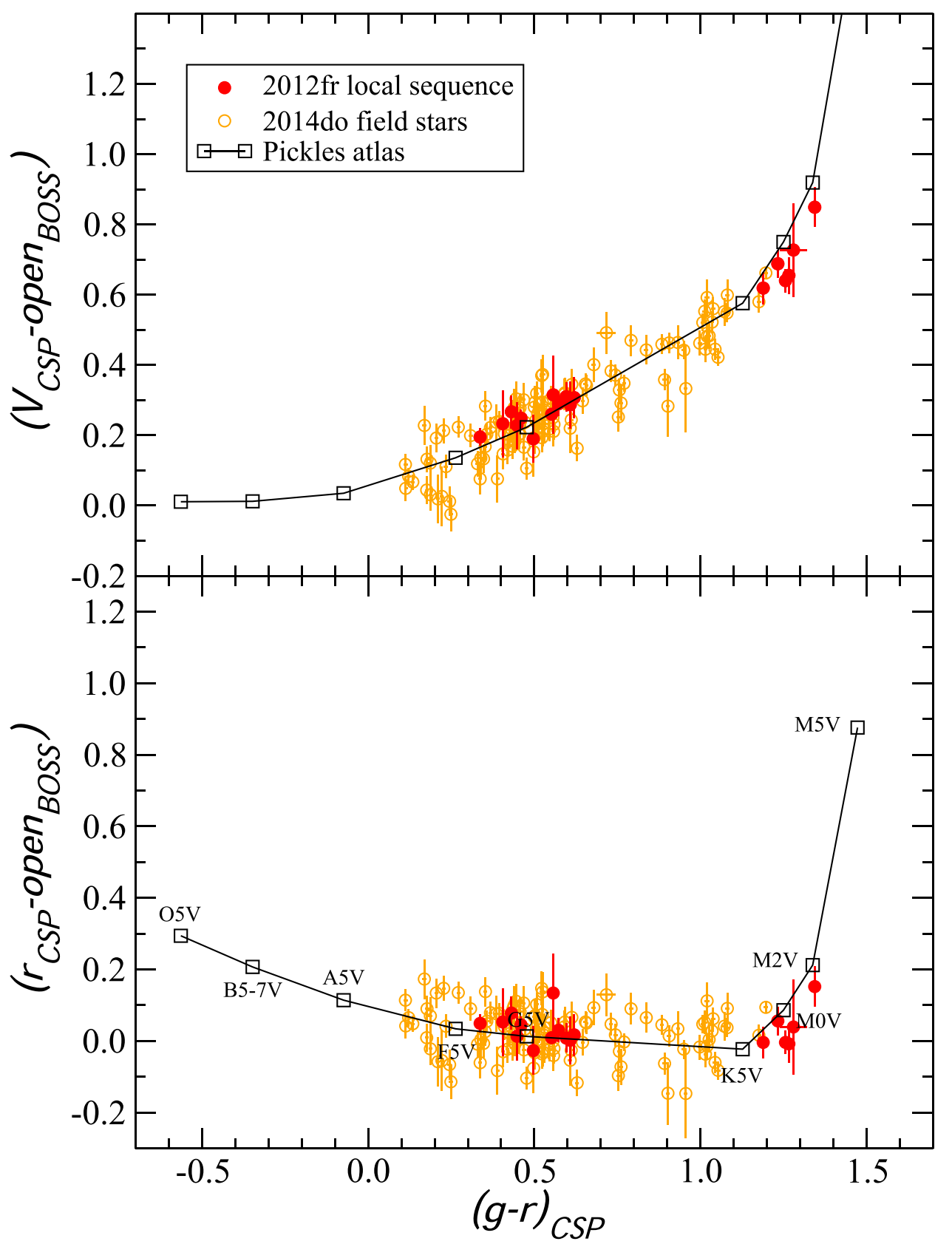

Figure 27. Color-color plots for converting CSP $V$ and $r$ magnitudes to the natural system magnitudes for the BOSS open filter. The black curves show synthetic photometry carried out using the Pickles (1998) stellar atlas. The red points correspond to observations of the local sequence stars in the field of SN $2012 \mathrm{fr}$.

3. Corrections are applied for Milky Way and host-galaxy dust reddening.

4. The resulting magnitudes in the natural system are transformed to $\mathrm{AB}$ magnitudes.

5. The AB magnitudes are then converted to monochromatic flux densities.

6. The bolometric flux at each phase is derived by integration of the flux densities via the trapezoidal rule.

7. Approximations are applied for the missing flux in the UV $\left(\lambda<\lambda_{u}\right)$ and infrared $\left(\lambda>\lambda_{H}\right)$ for each phase.

8 . The resulting bolometric flux versus time is converted to luminosity assuming the Cepheid-based distance modulus for the host-galaxy, NGC 1365.
Note that the K-corrections can be neglected because the redshift of the host-galaxy, NGC 1365, is very small $\left(z_{\text {helio }}=0.005457\right)$.

We used the smooth curve Gaussian process fitting to match the photometry in the NIR $Y J H$ bands to the phases of the optical photometry. The magnitudes were interpolated for the few nights when optical observations were not obtained. Next, the dust extinction from the Milky Way in the direction of SN 2012fr was corrected by subtracting the absorption values given in the third column of Table 11. For most of the filters, these were taken directly from NED. For the $Y$-band, these were calculated using Fitzpatrick's (1999) galactic-reddening law. Finally, the correction by host-galaxy reddening (see 


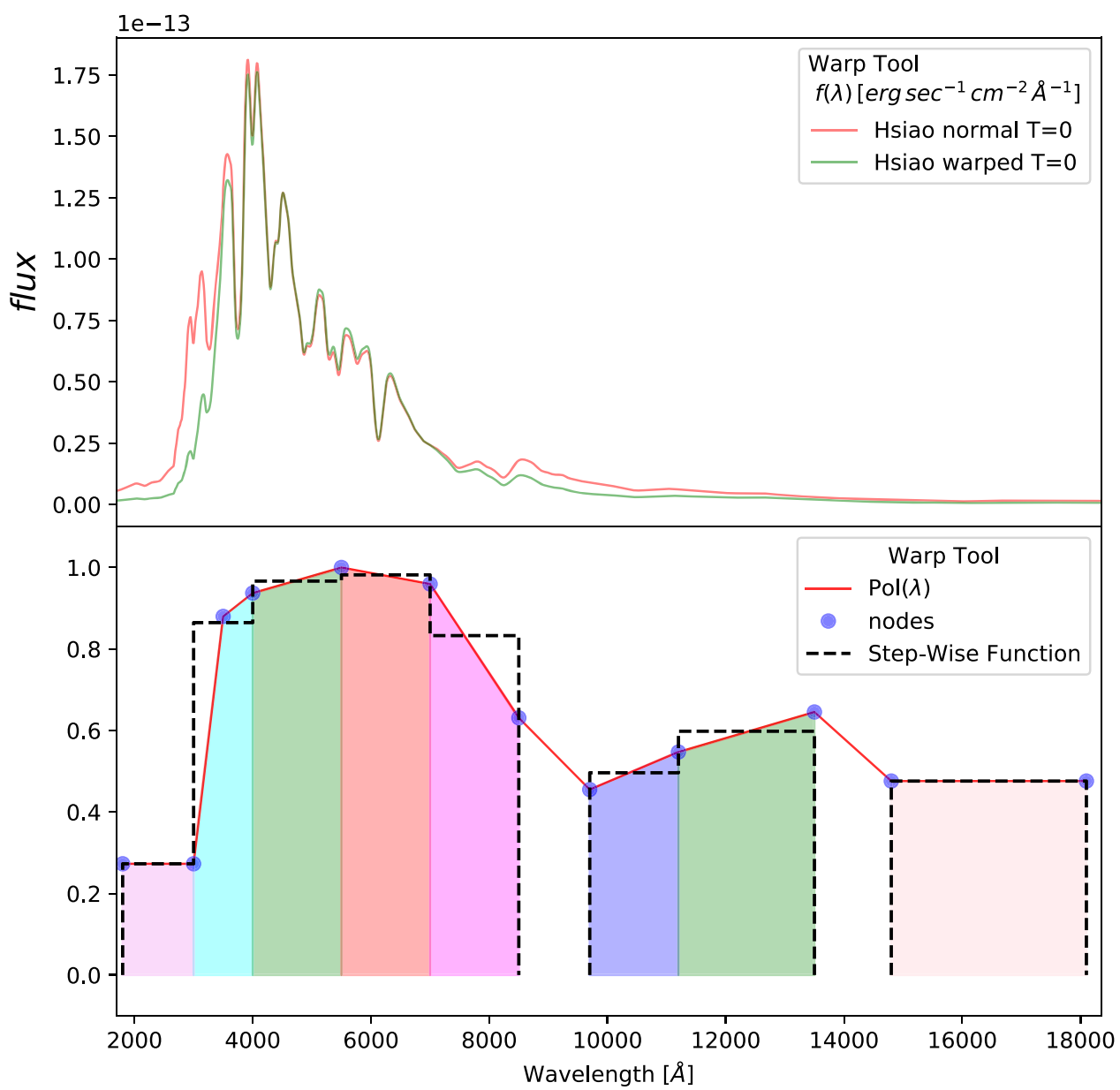

Figure 28. An example of the two-step process that was devised to match the Hsiao et al. (2007) spectral template to the observed CSP photometry for the same epoch. The dashed line in the lower panel shows the first iteration consisting of a step-wise function, $P(\lambda)$, as calculated from Equation (10). In the second iteration, a continuous piece-wise function is derived from the step-wise function by forcing the value of the nodes of the $g$-band bin to have the slope determined by the measured step-wise values of $u$ and $r$. The nodes for the remaining filters are then calculated from their step-wise values and the shaded regions correspond to the eight bins. The Hsiao et al. template spectrum is then multiplied by the final piece-wise function, as shown in the upper panel.

Section 3.2) was performed by subtracting the values given in the final column of Table 11 .

The $u v m 2, u, B, g, V, r, i, Y, J$, and $H$ photometry were then converted to $\mathrm{AB}$ magnitudes using the offsets given in the final column of Table 11. Once these are applied, the flux in each band is approximated by:

$$
f_{\nu}(X)=10^{-0.4\left(m_{\mathrm{AB}}+48.6\right)} \mathrm{erg} \mathrm{s}^{-1} \mathrm{~cm}^{-2} \mathrm{~Hz}^{-1} .
$$

The $u$-to- $H$ flux was then integrated using the trapezoidal rule. The flux beyond the $H$ band was estimated by assuming that it follows the Rayleigh-Jeans law. This leads to the following expression for the integrated flux at wavelengths longward of $\lambda_{H}$ :

$$
f\left(\lambda>\lambda_{H}\right)=f_{H} \lambda_{H} \frac{1}{5} .
$$

We estimate the UV contribution between 1800 and $3000 \AA$ assuming the the SED is flat there and we then estimate the contribution between $3000 \AA$ and $\lambda_{u}(3500 \AA$ ) by imposing the condition that the flux falls linearly to the flux estimated for uvm2-band from $\lambda_{u}$. The flux below $1800 \AA$ is assumed to be zero. Hence,

$$
\begin{aligned}
f\left(\lambda<\lambda_{u}\right)= & f_{u v m 2}(3000-1800 \AA) \\
& +\frac{1}{2}\left(f_{u}+f_{u v m 2}\right)(3500-3000 \AA) .
\end{aligned}
$$

This assumption fits well with the fall off of the flux at wavelengths below the $u$-band that are typically observed in UV spectra of SNe Ia (e.g., see Foley et al. 2016).

Finally, to calculate the bolometric luminosity in absolute terms, we assumed the Freedman et al. (2001) distance modulus of $\mu=31.27 \pm 0.05$ mag as derived from Cepheid variable observations.

\section{B.2. Spectral Template Fitting}

Our second method to estimate the bolometric luminosity takes the spectral template for each phase from Hsiao et al. (2007) and then multiplies it by a function $P(\lambda)$ such that the synthetic photometry measured on the template exactly matches the real photometry. This method is limited to $t-t_{B_{\max }}=+79$ because this is the last epoch for the spectral template. The steps can be summarized as follows: 


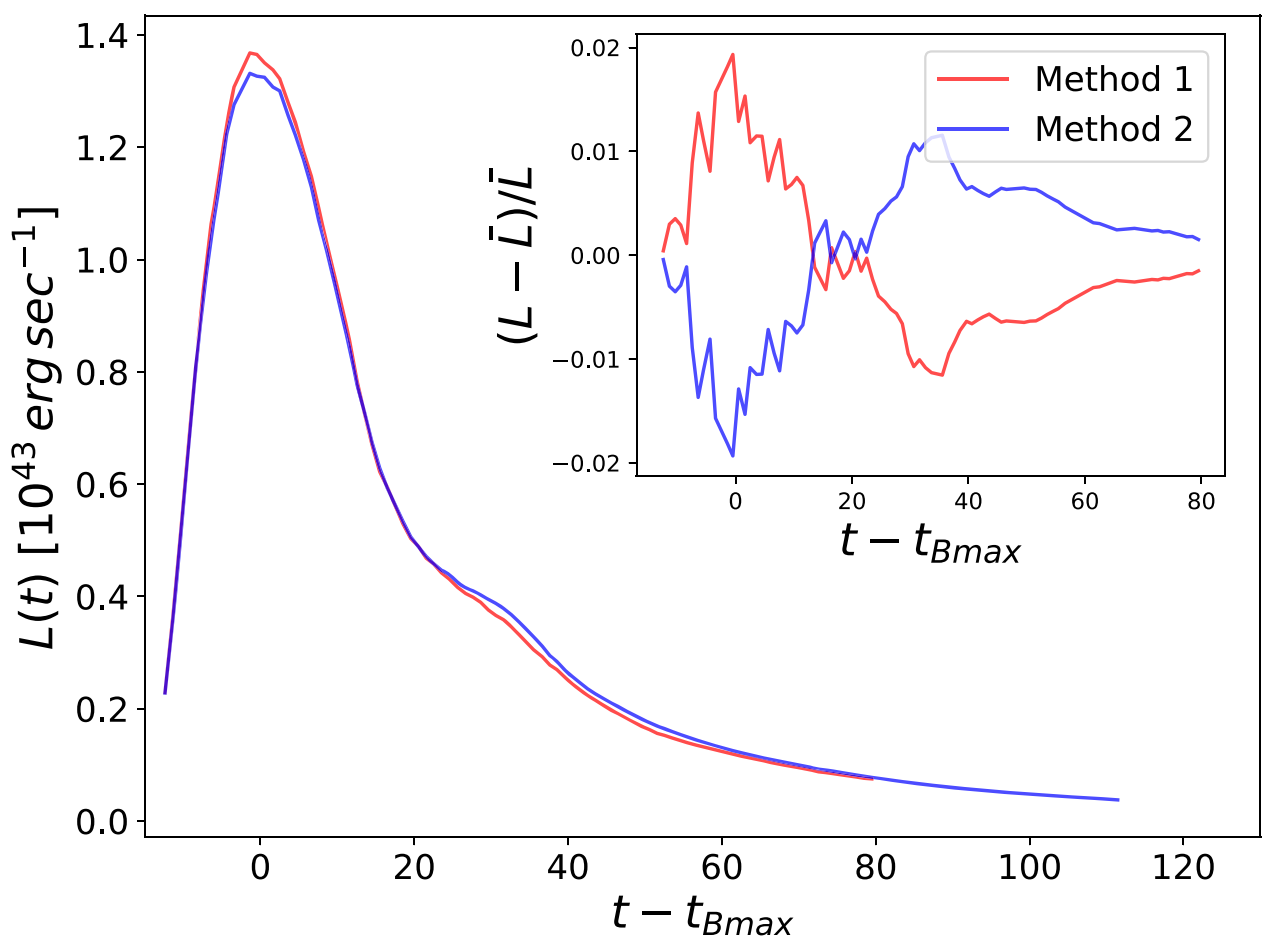

Figure 29. Bolometric light curves calculated with the photometric trapezoidal integration (Method 1) and the spectral template fitting (Method 2) techniques. The inset plot shows luminosity differences of both methods with respect to the average of the two methods.

1. The NIR $Y J H$ magnitudes are interpolated to match the optical phase coverage.

2. The optical magnitudes are interpolated for nights where observations were not available.

3. Corrections are applied for Milky Way and host-galaxy dust reddening.

4. For each photometry epoch, the Hsiao et al. spectrum corresponding to the phase that is selected.

5. The template spectrum for each epoch is then matched in flux to the photometry via an iterative procedure:

(a) First iteration. The spectrum is divided into wavelength bins corresponding to the non-overlapping filters $u v m 2, u, g, r, i, Y, J$, and $H$ which, in practice, cover almost all the UV-to-NIR wavelength domain, except for two gaps: one between $i$ and $Y$, and other between $J$ and $H$. Then, a step-wise function $P(\lambda)$ is fitted such that:

$m_{X}=-2.5 \log _{10} \int S_{X} F_{\lambda} \lambda P_{\lambda}(\lambda) d \lambda+z p_{X}$

where $X$ is the filter, $m_{X}$ is the reddening-corrected magnitude of the SN for that filter, $S_{X}$ is the transmission function of the filter, $F_{\lambda}$ is the Hsiao et al. spectrum, and $z p_{X}$ is the zero point that was previously adjusted to match our system.

(b) Second iteration. The step-wise function that was calculated in Step 5 is now converted to a piece-wise function (or polygonal line) with nodes at the blue and red limits of each filter, except for the $u$-band where we added an extra node at the effective wavelength to account for the rapid change of flux that occurs across this filter. The values of the nodes for the $g$-band bin are then imposed to ensure that the slope is determined by the step-wise values of $u$ and $r$ that were measured in the previous step. The nodes of $Y$ and $J$ also adopt the slope of the two corresponding step-wise values measured in the previous step. This smooths the final function $P(\lambda)$. All of the other nodes are then determined and may be calculated via Equation (10). Figure 28 shows an example of this two-step procedure.

6. The modified Hsiao et al. spectra, $W(\lambda)=P(\lambda) F_{\lambda}$, are then integrated between the effective wavelengths $\lambda_{u}$ and $\lambda_{H}$ to calculate the bolometric flux at each phase.

7. For the infrared flux at wavelengths longward of $\lambda_{H}$, a Rayleigh-Jeans law is again assumed:

$$
f\left(\lambda>\lambda_{H}\right)=f_{H} \lambda_{H} \frac{1}{5}
$$

8. Finally, the flux is converted into luminosity by assuming the Cepheid-based distance modulus to NGC 1365.

The upper panel of Figure 29 displays the final bolometric light curves calculated using the photometric trapezoidal integration and spectral template fitting methods. In the lower panel of the figure, the ratio of each light curve to the average of the two is plotted versus phase with respect to the epoch of 
Table 12

Bolometric Luminosity of SN 2012fr

\begin{tabular}{|c|c|c|c|c|c|c|c|c|c|}
\hline $\begin{array}{l}\text { JD } \\
\text { (days) }\end{array}$ & $\begin{array}{l}\begin{array}{l}\text { Phase } \\
\text { (days) }\end{array}\end{array}$ & $\begin{array}{c}L_{1}(\text { Total }) \\
\left(10^{43} \mathrm{erg} \mathrm{s}^{-1}\right)\end{array}$ & $\begin{array}{c}L_{\mathrm{UVOIR}} \\
(\%)\end{array}$ & $\begin{array}{l}L_{\text {uvm } 2} \\
(\%)\end{array}$ & $\begin{array}{c}L_{\left(\lambda>\lambda_{H}\right)} \\
(\%)\end{array}$ & $\begin{array}{c}L_{2} \text { (Total) } \\
\left(10^{43} \mathrm{erg} \mathrm{s}^{-1}\right)\end{array}$ & $\begin{array}{c}L_{\mathrm{UVOIR}} \\
(\%)\end{array}$ & $\begin{array}{l}L_{\text {uvm } 2} \\
(\%)\end{array}$ & $\begin{array}{c}L_{\left(\lambda>\lambda_{H}\right)} \\
(\%)\end{array}$ \\
\hline 2456230.70 & -12.40 & $0.228(021)$ & 98.15 & 0.30 & 1.85 & $0.229(022)$ & 97.51 & 0.59 & 1.91 \\
\hline 2456231.75 & -11.35 & $0.362(035)$ & 98.54 & 0.30 & 1.46 & $0.368(037)$ & 97.95 & 0.57 & 1.48 \\
\hline 2456232.75 & -10.35 & $0.512(050)$ & 98.77 & 0.32 & 1.23 & $0.519(053)$ & 98.15 & 0.59 & 1.26 \\
\hline 2456233.70 & -09.40 & $0.655(066)$ & 98.90 & 0.35 & 1.10 & $0.661(068)$ & 98.21 & 0.65 & 1.14 \\
\hline 2456234.70 & -08.40 & $0.809(082)$ & 99.00 & 0.39 & 1.00 & $0.812(085)$ & 98.24 & 0.73 & 1.03 \\
\hline 2456235.67 & -07.43 & $0.927(095)$ & 99.05 & 0.44 & 0.95 & $0.945(100)$ & 98.22 & 0.82 & 0.96 \\
\hline 2456236.69 & -06.41 & $1.036(107)$ & 99.09 & 0.52 & 0.91 & $1.063(114)$ & 98.17 & 0.92 & 0.91 \\
\hline 2456237.60 & -05.50 & $1.117(115)$ & 99.12 & 0.58 & 0.88 & $1.139(122)$ & 98.11 & 1.00 & 0.88 \\
\hline 2456238.72 & -04.38 & $1.224(127)$ & 99.18 & 0.66 & 0.82 & $1.240(134)$ & 98.03 & 1.13 & 0.84 \\
\hline 2456239.67 & -03.43 & $1.276(133)$ & 99.21 & 0.73 & 0.79 & $1.307(142)$ & 97.91 & 1.29 & 0.81 \\
\hline 2456241.73 & -01.37 & $1.332(140)$ & 99.26 & 0.89 & 0.74 & $1.368(151)$ & 97.51 & 1.73 & 0.76 \\
\hline 2456242.64 & -00.46 & $1.327(139)$ & 99.27 & 0.96 & 0.73 & $1.365(151)$ & 97.32 & 1.94 & 0.74 \\
\hline 2456243.63 & +00.53 & $1.325(139)$ & 99.30 & 1.02 & 0.70 & $1.350(149)$ & 97.20 & 2.08 & 0.72 \\
\hline 2456244.73 & +01.63 & $1.307(137)$ & 99.32 & 1.07 & 0.68 & $1.338(148)$ & 97.13 & 2.18 & 0.69 \\
\hline 2456245.61 & +02.51 & $1.300(137)$ & 99.34 & 1.09 & 0.66 & $1.322(147)$ & 97.09 & 2.24 & 0.66 \\
\hline 2456246.71 & +03.61 & $1.256(132)$ & 99.34 & 1.12 & 0.66 & $1.279(142)$ & 97.05 & 2.31 & 0.64 \\
\hline 2456247.65 & +04.55 & $1.222(129)$ & 99.35 & 1.13 & 0.65 & $1.245(138)$ & 97.05 & 2.33 & 0.62 \\
\hline 2456248.71 & +05.61 & $1.178(124)$ & 99.35 & 1.12 & 0.65 & $1.192(133)$ & 97.01 & 2.38 & 0.61 \\
\hline 2456249.73 & +06.63 & $1.129(119)$ & 99.35 & 1.11 & 0.65 & $1.148(128)$ & 96.97 & 2.42 & 0.61 \\
\hline 2456250.67 & +07.57 & $1.070(112)$ & 99.33 & 1.11 & 0.67 & $1.093(122)$ & 96.89 & 2.48 & 0.63 \\
\hline 2456251.71 & +08.61 & $1.016(106)$ & 99.32 & 1.09 & 0.68 & $1.029(115)$ & 96.79 & 2.54 & 0.67 \\
\hline 2456252.71 & +09.61 & $0.961(100)$ & 99.29 & 1.06 & 0.71 & $0.974(108)$ & 96.83 & 2.50 & 0.67 \\
\hline 2456253.64 & +10.54 & $0.903(094)$ & 99.26 & 1.04 & 0.74 & $0.918(102)$ & 96.97 & 2.39 & 0.64 \\
\hline 2456254.66 & +11.56 & $0.845(088)$ & 99.22 & 1.02 & 0.78 & $0.858(095)$ & 97.05 & 2.29 & 0.66 \\
\hline 2456255.67 & +12.57 & $0.777(081)$ & 99.15 & 1.01 & 0.85 & $0.783(086)$ & 97.00 & 2.27 & 0.73 \\
\hline 2456256.68 & +13.58 & $0.726(075)$ & 99.10 & 0.98 & 0.90 & $0.723(079)$ & 96.98 & 2.21 & 0.82 \\
\hline 2456257.60 & +14.50 & $0.675(069)$ & 99.02 & 0.96 & 0.98 & $0.671(073)$ & 96.94 & 2.18 & 0.88 \\
\hline 2456258.61 & +15.51 & $0.629(064)$ & 98.94 & 0.93 & 1.06 & $0.622(067)$ & 97.09 & 1.98 & 0.93 \\
\hline 2456259.59 & +16.49 & $0.593(060)$ & 98.87 & 0.89 & 1.13 & $0.595(063)$ & 97.14 & 1.88 & 0.98 \\
\hline 2456261.62 & +18.52 & $0.534(053)$ & 98.70 & 0.81 & 1.30 & $0.530(055)$ & 97.32 & 1.54 & 1.15 \\
\hline 2456262.65 & +19.55 & $0.506(049)$ & 98.60 & 0.78 & 1.40 & $0.503(051)$ & 97.34 & 1.42 & 1.24 \\
\hline 2456263.65 & +20.55 & $0.488(047)$ & 98.51 & 0.74 & 1.49 & $0.489(049)$ & 97.37 & 1.32 & 1.30 \\
\hline 2456264.66 & +21.56 & $0.471(044)$ & 98.43 & 0.70 & 1.57 & $0.468(046)$ & 97.36 & 1.25 & 1.39 \\
\hline 2456265.62 & +22.52 & $0.459(043)$ & 98.35 & 0.66 & 1.65 & $0.458(045)$ & 97.38 & 1.16 & 1.46 \\
\hline 2456266.63 & +23.53 & $0.447(041)$ & 98.27 & 0.63 & 1.73 & $0.442(042)$ & 97.38 & 1.05 & 1.57 \\
\hline 2456267.65 & +24.55 & $0.439(039)$ & 98.20 & 0.59 & 1.80 & $0.431(041)$ & 97.39 & 0.94 & 1.67 \\
\hline 2456268.74 & +25.64 & $0.425(038)$ & 98.11 & 0.57 & 1.89 & $0.416(038)$ & 97.36 & 0.85 & 1.79 \\
\hline 2456269.78 & +26.68 & $0.416(036)$ & 98.03 & 0.54 & 1.97 & $0.405(037)$ & 97.32 & 0.80 & 1.89 \\
\hline 2456270.74 & +27.64 & $0.410(035)$ & 97.99 & 0.52 & 2.01 & $0.399(036)$ & 97.27 & 0.81 & 1.92 \\
\hline 2456271.74 & +28.64 & $0.403(034)$ & 97.93 & 0.50 & 2.07 & $0.390(034)$ & 97.19 & 0.84 & 1.97 \\
\hline 2456272.74 & +29.64 & $0.395(033)$ & 97.88 & 0.48 & 2.12 & $0.376(033)$ & 97.09 & 0.88 & 2.03 \\
\hline 2456273.71 & +30.61 & $0.388(032)$ & 97.84 & 0.47 & 2.16 & $0.366(031)$ & 97.00 & 0.91 & 2.08 \\
\hline 2456274.69 & +31.59 & $0.379(030)$ & 97.80 & 0.46 & 2.20 & $0.359(030)$ & 96.93 & 0.91 & 2.16 \\
\hline 2456275.72 & +32.62 & $0.368(029)$ & 97.76 & 0.45 & 2.24 & $0.346(029)$ & 96.81 & 0.92 & 2.27 \\
\hline 2456276.75 & +33.65 & $0.354(028)$ & 97.71 & 0.45 & 2.29 & $0.332(027)$ & 96.67 & 0.94 & 2.39 \\
\hline 2456278.61 & +35.51 & $0.328(025)$ & 97.62 & 0.45 & 2.38 & $0.305(025)$ & 96.41 & 0.99 & 2.61 \\
\hline 2456279.74 & +36.64 & $0.312(024)$ & 97.56 & 0.46 & 2.44 & $0.293(024)$ & 96.33 & 0.99 & 2.68 \\
\hline 2456280.73 & +37.63 & $0.295(022)$ & 97.50 & 0.47 & 2.50 & $0.278(022)$ & 96.20 & 1.01 & 2.79 \\
\hline 2456281.64 & +38.54 & $0.284(022)$ & 97.48 & 0.48 & 2.52 & $0.270(022)$ & 96.15 & 1.01 & 2.84 \\
\hline 2456282.76 & +39.66 & $0.268(020)$ & 97.43 & 0.50 & 2.57 & $0.255(020)$ & 96.06 & 1.02 & 2.92 \\
\hline 2456283.68 & +40.58 & $0.257(020)$ & 97.42 & 0.51 & 2.58 & $0.244(020)$ & 95.99 & 1.01 & 3.00 \\
\hline 2456284.68 & +41.58 & $0.246(019)$ & 97.41 & 0.52 & 2.59 & $0.234(019)$ & 95.95 & 1.00 & 3.05 \\
\hline 2456285.65 & +42.55 & $0.235(018)$ & 97.41 & 0.53 & 2.59 & $0.224(018)$ & 95.90 & 0.99 & 3.11 \\
\hline 2456286.65 & +43.55 & $0.227(017)$ & 97.43 & 0.54 & 2.57 & $0.215(017)$ & 95.88 & 0.98 & 3.15 \\
\hline 2456287.75 & +44.65 & $0.218(017)$ & 97.45 & 0.55 & 2.55 & $0.206(017)$ & 95.84 & 0.98 & 3.18 \\
\hline 2456288.73 & +45.63 & $0.210(016)$ & 97.48 & 0.55 & 2.52 & $0.197(016)$ & 95.79 & 1.02 & 3.19 \\
\hline 2456289.60 & +46.50 & $0.204(016)$ & 97.51 & 0.56 & 2.49 & $0.192(016)$ & 95.82 & 1.03 & 3.15 \\
\hline 2456292.65 & +49.55 & $0.182(014)$ & 97.57 & 0.59 & 2.43 & $0.169(014)$ & 95.62 & 1.21 & 3.17 \\
\hline 2456293.66 & +50.56 & $0.176(014)$ & 97.60 & 0.60 & 2.40 & $0.163(014)$ & 95.60 & 1.25 & 3.15 \\
\hline 2456294.68 & +51.58 & $0.169(014)$ & 97.61 & 0.61 & 2.39 & $0.156(013)$ & 95.52 & 1.31 & 3.16 \\
\hline 2456295.63 & +52.53 & $0.165(013)$ & 97.65 & 0.61 & 2.35 & $0.153(013)$ & 95.53 & 1.35 & 3.13 \\
\hline 2456296.60 & +53.50 & $0.160(013)$ & 97.67 & 0.61 & 2.33 & $0.148(013)$ & 95.54 & 1.37 & 3.09 \\
\hline 2456297.67 & +54.57 & $0.154(013)$ & 97.69 & 0.62 & 2.31 & $0.144(012)$ & 95.55 & 1.39 & 3.06 \\
\hline 2456298.53 & +55.43 & $0.150(012)$ & 97.70 & 0.62 & 2.30 & $0.140(012)$ & 95.57 & 1.39 & 3.04 \\
\hline
\end{tabular}


Table 12

(Continued)

\begin{tabular}{|c|c|c|c|c|c|c|c|c|c|}
\hline $\begin{array}{l}\text { JD } \\
\text { (days) }\end{array}$ & $\begin{array}{l}\text { Phase } \\
\text { (days) }\end{array}$ & $\begin{array}{c}L_{1}(\text { Total }) \\
\left(10^{43} \mathrm{erg} \mathrm{s}^{-1}\right)\end{array}$ & $\begin{array}{l}L_{\mathrm{UVOIR}} \\
(\%)\end{array}$ & $\begin{array}{l}L_{\text {uvm } 2} \\
(\%)\end{array}$ & $\begin{array}{l}L_{\left(\lambda>\lambda_{H}\right)} \\
\quad(\%)\end{array}$ & $\begin{array}{c}L_{2} \text { (Total) } \\
\left(10^{43} \mathrm{erg} \mathrm{s}^{-1}\right)\end{array}$ & $\begin{array}{l}L_{\mathrm{UVOIR}} \\
(\%)\end{array}$ & $\begin{array}{l}L_{\text {uvm } 2} \\
(\%)\end{array}$ & $\begin{array}{l}L_{\left(\lambda>\lambda_{H}\right)} \\
(\%)\end{array}$ \\
\hline 2456299.69 & +56.59 & $0.145(012)$ & 97.72 & 0.62 & 2.28 & $0.136(012)$ & 95.57 & 1.42 & 3.01 \\
\hline 2456304.54 & +61.44 & $0.126(011)$ & 97.77 & 0.63 & 2.23 & $0.119(011)$ & 95.62 & 1.49 & 2.90 \\
\hline 2456308.55 & +65.45 & $0.112(010)$ & 97.80 & 0.63 & 2.20 & $0.107(010)$ & 95.76 & 1.49 & 2.75 \\
\hline 2456311.61 & +68.51 & $0.104(009)$ & 97.85 & 0.63 & 2.15 & $0.099(009)$ & 95.92 & 1.50 & 2.58 \\
\hline 2456314.59 & +71.49 & $0.096(009)$ & 97.91 & 0.63 & 2.09 & $0.091(008)$ & 96.07 & 1.50 & 2.42 \\
\hline 2456317.58 & +74.48 & $0.089(008)$ & 97.99 & 0.63 & 2.01 & $0.084(008)$ & 96.34 & 1.52 & 2.14 \\
\hline 2456318.59 & +75.49 & $0.087(008)$ & 98.02 & 0.63 & 1.98 & $0.082(008)$ & 96.38 & 1.52 & 2.10 \\
\hline 2456320.57 & +77.47 & $0.082(007)$ & 98.08 & 0.63 & 1.92 & $0.079(007)$ & 96.47 & 1.53 & 2.00 \\
\hline 2456321.56 & +78.46 & $0.080(007)$ & 98.11 & 0.64 & 1.89 & $0.076(007)$ & 96.50 & 1.54 & 1.96 \\
\hline 2456322.59 & +79.49 & $0.078(007)$ & 98.16 & 0.63 & 1.84 & $0.075(007)$ & 96.58 & 1.53 & 1.89 \\
\hline 2456323.62 & +80.52 & $0.076(007)$ & 98.20 & 0.63 & 1.80 & $\ldots$ & $\ldots$ & $\ldots$ & $\ldots$ \\
\hline 2456328.57 & +85.47 & $0.066(006)$ & 98.36 & 0.66 & 1.64 & $\ldots$ & $\ldots$ & $\ldots$ & $\ldots$ \\
\hline 2456329.56 & +86.46 & $0.065(006)$ & 98.41 & 0.66 & 1.59 & $\ldots$ & $\ldots$ & $\ldots$ & $\ldots$ \\
\hline 2456330.55 & +87.45 & $0.063(006)$ & 98.43 & 0.67 & 1.57 & $\ldots$ & $\ldots$ & $\ldots$ & $\ldots$ \\
\hline 2456331.53 & +88.43 & $0.062(006)$ & 98.47 & 0.67 & 1.53 & $\ldots$ & $\ldots$ & $\ldots$ & $\ldots$ \\
\hline 2456332.52 & +89.42 & $0.061(006)$ & 98.51 & 0.67 & 1.49 & $\ldots$ & $\ldots$ & $\ldots$ & $\ldots$ \\
\hline 2456336.56 & +93.46 & $0.056(005)$ & 98.62 & 0.69 & 1.38 & $\ldots$ & $\ldots$ & $\ldots$ & $\ldots$ \\
\hline 2456337.57 & +94.47 & $0.054(005)$ & 98.62 & 0.71 & 1.38 & $\ldots$ & $\ldots$ & $\ldots$ & $\ldots$ \\
\hline 2456340.57 & +97.47 & $0.051(005)$ & 98.69 & 0.71 & 1.31 & $\ldots$ & $\ldots$ & $\ldots$ & $\ldots$ \\
\hline 2456342.60 & +99.50 & $0.049(005)$ & 98.72 & 0.73 & 1.28 & $\ldots$ & $\ldots$ & $\ldots$ & $\ldots$ \\
\hline 2456343.58 & +100.48 & $0.048(005)$ & 98.72 & 0.74 & 1.28 & $\ldots$ & $\ldots$ & $\ldots$ & $\ldots$ \\
\hline 2456345.57 & +102.47 & $0.046(004)$ & 98.74 & 0.75 & 1.26 & $\ldots$ & $\ldots$ & $\ldots$ & $\ldots$ \\
\hline 2456346.58 & +103.48 & $0.045(004)$ & 98.75 & 0.76 & 1.25 & $\ldots$ & $\ldots$ & $\ldots$ & $\ldots$ \\
\hline 2456349.58 & +106.48 & $0.042(004)$ & 98.76 & 0.79 & 1.24 & $\ldots$ & $\ldots$ & $\ldots$ & $\ldots$ \\
\hline
\end{tabular}

$L_{(1,2)}$ : Bolometric luminosity in the wavelength range $\lambda>1800 \AA$.

$L_{\text {UVOIR }}$ : Luminosity in the wavelength range $3000<\lambda<16600 \AA$.

$L_{u v m 2}$ : Luminosity in the wavelength range $1800<\lambda<3000 \AA$.

$L_{\lambda>\lambda_{H}}$ : Luminosity in the wavelength range $\lambda>16600 \AA$.

Note. The phase is relative to $t_{B_{\max }}=\mathrm{JD} 2456243.1$. $L_{\mathrm{UVOIR}}$ is the luminosity measured from the flux of $2012 \mathrm{fr}$ through $u B g$ VriYJH-bands photometry.

$t_{B_{\max }}$. It can be seen that the two methods produce light curves that are consistent at the $\pm 5 \%$ level. The spectral template method yields a higher luminosity at phases before $t-t_{B_{\max }} \sim 30$ and a lower luminosity at later epochs. The final bolometric light curves for the two methods are given in Table 12.

\section{ORCID iDs}

Carlos Contreras (ib https://orcid.org/0000-0001-6293-9062 M. M. Phillips (iD https://orcid.org/0000-0003-2734-0796 Christopher R. Burns (i) https://orcid.org/0000-00034625-6629

Anthony L. Piro (1) https://orcid.org/0000-0001-6806-0673 Maximilian D. Stritzinger (iD https://orcid.org/0000-00025571-1833

Peter J. Brown (1D https://orcid.org/0000-0001-6272-5507

Peter E. Nugent (ib https://orcid.org/0000-0002-3389-0586 Damien Turpin (i) https://orcid.org/0000-0003-1835-1522
Eric Y. Hsiao (ib https://orcid.org/0000-0003-1039-2928

Nidia Morrell (iD https://orcid.org/0000-0003-2535-3091

Kevin Krisciunas (ib https://orcid.org/0000-0002-6650-694X

Brad E. Tucker (i) https://orcid.org/0000-0002-4283-5159

E. Baron (10) https://orcid.org/0000-0001-5393-1608

P. Hoeflich (iD https://orcid.org/0000-0002-4338-6586

Brian Schmidt (iD https://orcid.org/0000-0002-8538-9195

\section{References}

Aldering, G., Knop, R., \& Nugent, P. 2000, AJ, 119, 2110 Arnett, W. D. 1982, ApJ, 253, 785

Baltay, C., Rabinowitz, D., Andrews, P., Bauer, A., et al. 2007, PASP, 119,1278

Baltay, C., Rabinowitz, D., Hadjiyska, E., et al. 2013, PASP, 125, 683 Benetti, S., Cappellaro, E., Mazzali, P. A., et al. 2005, ApJ, 623, 1011 Betoule, M., Kessler, R., Guy, J., et al. 2014, A\&A, 568, A22 Blondin, S., Matheson, T., Kirshner, R. P., et al. 2012, AJ, 143, 126 Bloom, J., Kasen, D., Shen, K., et al. 2012, ApJL, 744, L17 Bohlin, R. C., Gordon, K. D., \& Tremblay, P.-E. 2014, PASP, 126, 711 
Branch, D. 1992, ApJ, 392, 35

Branch, D., Dang, L. C., \& Baron, E. 2009, PASP, 121, 238

Branch, D., Dang, L. C., Nicholas Hall, K., et al. 2006, PASP, 118, 560

Branch, D., Fisher, A., \& Nugent, P. 1993, AJ, 106, 2383

Branch, D., Thomas, R. C., Baron, E., et al. 2004, ApJ, 606, 413

Breeveld, A. A., Landsman, W., Holland, S. T., et al. 2011, in AIP Conf. Ser. 1358, Gamma Ray Bursts 2010, ed. J. E. McEnery, J. L. Racusin, \& N. Gehrels (Melville, NY: AIP), 373

Brown, P. J., Breeveld, A., Roming, P. W. A., et al. 2016, AJ, 152, 102 Brown, P. J., Dawson, K. S., de Pasquale, M., et al. 2012, ApJ, 753, 22 Brown, P. J., Holland, S. T., Immler, S., et al. 2009, ApJ, 137, 4517 Burns, C. R., Stritzinger, M., Phillips, M. M., et al. 2011, AJ, 141, 19 Burns, C. R., Stritzinger, M., Phillips, M. M., et al. 2014, ApJ, 789, 32 Candia, P., Krisciunas, K., Suntzeff, N. B., et al. 2003, PASP, 115, 277 Castelli, F., \& Kurucz, R. L. 2003, in IAU Smp. 210, Modelling of Stellar Atmospheres, ed. N. Piskunov, W. W. Weiss, \& D. F. Gray (San Francisco, CA: ASP), A20

Childress, M. J., Hillier, D. J., Seitenzahl, I., et al. 2015, MNRAS, 454, 3816

Childress, M. J., Scalzo, R. A., Sim, S. A., et al. 2013, ApJ, 770, 29

Childress, M. J., Zhou, G., Tucker, B., et al. 2012, CBET, 3275

Conley, A., Howell, D. A., Howes, A., et al. 2006, AJ, 132, 1707

Contreras, C., Hamuy, M., Phillips, M. M., et al. 2010, AJ, 139, 519

Dan, M., Rosswog, S., Guillochon, J., \& Ramirez-Ruiz, E. 2012, MNRAS, 422, 2417

Filippenko, A. V., Richmond, M. W., Branch, D., et al. 1992, AJ, 104, 1543

Fink, M., Röpke, F. K., Hillebrandt, W., et al. 2010, A\&A, 514, A53

Finlator, K., Ivezić, Ž., Fan, X., et al. 2000, AJ, 120, 2615

Firth, R. E., Sullivan, M., Gal-Yam, A., et al. 2015, MNRAS, 446, 3895

Fitzpatrick, E. L. 1999, PASP, 111, 63

Folatelli, G., Morrell, N., Phillips, M., et al. 2013, ApJ, 773, 53

Folatelli, G., Phillips, M., Burns, C. R., et al. 2010, AJ, 139, 120

Foley, R. J., Challis, P., Filippenko, A. V., et al. 2012, ApJ, 744, 38

Foley, R. J., Pan, Y.-C., Brown, P., et al. 2016, MNRAS, 461, 1308

Foley, R. J., Sanders, N. E., \& Kirshner, R. P. 2011, ApJ, 742, 89

Freedman, W., Madore, B., Gibson, B. K., et al. 2001, AJ, 553, 47

Fryer, C. L., Ruiter, A. J., Belczynski, K., et al. 2010, ApJ, 725, 296

Fukugita, M., Ichikawa, T., Gunn, J. E., Shimasaku, K., \& Schneider, D. P. 1996, AJ, 111, 1748

Ganeshalingam, M., Li, W., \& Filippenko, A. 2011, MNRAS, 416, 2607

Garavini, G., Folatelli, G., Goobar, A., et al. 2004, AJ, 128, 387

Goldhaber, G., Groom, D. E., Kim, A., et al. 2001, ApJ, 558, 359

González-Gaitán, S., Conley, A., Bianco, F. B., et al. 2012, ApJ, 745, 44

Goobar, A., Kromer, M., Siverd, R., et al. 2015, ApJ, 799, 106

Graham, M. L., Kumar, S., Hosseinzadeh, G., et al. 2017, MNRAS, 472, 3437

Guillochon, J., Dan, M., Ramirez-Ruiz, E., \& Rosswog, S. 2010, ApJL, 709, L64

Hamuy, M., Folatelli, G., Morrell, N. I., et al. 2006, PASP, 118, 2

Hayden, B. T., Garnavich, P. M., Kessler, R., et al. 2010, ApJ, 712, 350

Hoeflich, P., Hsiao, E. Y., Ashall, C., et al. 2017, ApJ, 846, 58

Höflich, P., Gerardy, C. L., Fesen, R. A., \& Sakai, S. 2002, ApJ, 568, 791

Höflich, P., \& Khokhlov, A. 1996, ApJ, 457, 500

Höflich, P., Khokhlov, A., Wheeler, J. C., et al. 1996, ApJL, 472, L81

Höflich, P., Krisciunas, K., Khokhlov, A. M., et al. 2010, ApJ, 710, 444

Hosseinzadeh, G., Sand, D. J., Valenti, S., et al. 2017, ApJL, 845, L11

Howell, D. A. 2011, NatCo, 2, 350

Hoyle, F., \& Fowler, W. A. 1960, ApJ, 132, 565

Hsiao, E. Y., Conley, A., Howell, D. A., et al. 2007, ApJ, 663, 1187

Hsiao, E. Y., Marion, G. H., Phillips, M. M., et al. 2013, ApJ, 766, 72

Jang, I. S., Hatt, D., Beaton, R. L., et al. 2018, ApJ, 852, 60

Jha, S., Riess, A., \& Kirshner, R. 2007, ApJ, 659, 122

Kasen, D. 2010, ApJ, 708, 1025

Kattner, S., Leonard, D., Burns, C. R., et al. 2012, PASP, 124, 114

Khokhlov, A., Mueller, E., \& Höflich, P. 1993, A\&A, 270, 223

Khokhlov, A. M. 1991, A\&A, 245, 114

Klotz, A. 2012, CBET, 3275

Klotz, A., Boër, M., Eysseric, J., et al. 2008, PASP, 120, 1298

Klotz, A., \& Conseil, E. 2012, ATel, 4523

Krisciunas, K., Contreras, C., Burns, C. R., et al. 2017, AJ, 154, 278

Kushnir, D., Katz, B., Dong, S., et al. 2013, ApJL, 778, L37

Landolt, A. U. 1992, AJ, 104, 340

Leibundgut, B., Kirshner, R. P., Phillips, M. M., et al. 1993, AJ, 105, 301

Li, W., Filippenko, A. V., Chornock, R., et al. 2003, PASP, 115, 453
Li, W., Filippenko, A. V., Gates, E., et al. 2001, PASP, 113, 1178

Li, W., Filippenko, A. V., Treffers, R. R., et al. 2001, ApJ, 546, 734

Lira, P. 1995, Masters Thesis, Univ. Chile

Maoz, D., Sharon, K., \& Gal-Yam, A. 2010, ApJ, 722, 1879

Marion, G. H., Vinko, J., Wheeler, J. C., et al. 2013, ApJ, 777, 40

Maund, J. R., Spyromilio, J., Höeflich, P. A., et al. 2013, MNRAS, 433, L20

Mazzali, P. A., Benetti, S., Altavilla, G., et al. 2005, ApJL, 623, L37

Mazzali, P. A., Sullivan, M., Filippenko, A. V., et al. 2015, MNRAS, 450, 2631

McWilliam, A. 1997, ARA\&A, 35, 503

Miller, A. A., Cao, Y., Piro, A. L., et al. 2018, ApJ, 852, 100

Morozova, V., Piro, A. L., Renzo, M., et al. 2015, ApJ, 814, 63

Munari, U., \& Zwitter, T. 1997, A\&A, 318, 269

Nadyozhin, D. K. 1994, ApJS, 92, 527

Noebauer, U. M., Kromer, M., Taubenberger, S., et al. 2017, MNRAS, 472, 2787

Nugent, P. E., Sullivan, M., Cenko, S. B., et al. 2011, Natur, 480, 344

Olling, R. P., Mushotzky, R., Shaya, E. J., et al. 2015, Natur, 521, 332

Pakmor, R., Kromer, M., Taubenberger, S., et al. 2012, ApJL, 747, L10

Parker, S. 2012, CBET, 3275

Pereira, R., Thomas, R. C., Aldering, G., et al. 2013, A\&A, 554, 27

Persson, S. E., Murphy, D. C., Krzeminski, W., et al. 1998, AJ, 116, 2475

Persson, S. E., Murphy, D. C., Smee, S., et al. 2013, PASP, 125, 654

Phillips, M. M. 1993, ApJL, 413, L105

Phillips, M. M., Lira, P., Suntzeff, N. B., et al. 1999, AJ, 118, 1766

Phillips, M. M., Simon, J. D., Morrell, N., et al. 2013, ApJ, 779, 38

Phillips, M. M., Wells, L. A., Suntzeff, N. B., et al. 1992, AJ, 103, 1632

Pickles, A. J. 1998, PASP, 110, 863

Piro, A. L., Chang, P., \& Weinberg, N. N. 2010, ApJ, 708, 598

Piro, A. L., \& Morozova, V. S. 2016, ApJ, 826, 96

Piro, A. L., \& Nakar, E. 2013, ApJ, 769, 67

Piro, A. L., \& Nakar, E. 2014, ApJ, 784, 85

Poole, T. S., Breeveld, A. A., Page, M. J., et al. 2008, MNRAS, 383, 627

Quimby, R., Höflich, P., \& Wheeler, J. C. 2007, ApJ, 666, 1083

Raskin, C., Timmes, F. X., Scannapieco, E., et al. 2009, MNRAS, 399, L156

Riess, A. G., Filippenko, A. V., Li, W., \& Schmidt, B. 1999, AJ, 118, 2668

Riess, A. G., Macri, L. M., Hoffmann, S. L., et al. 2016, ApJ, 826, 56

Rosswog, S., Kasen, D., Guillochon, J., \& Ramirez-Ruiz, E. 2009, ApJL, 705, L128

Ruiz-Lapuente, P., Cappellaro, E., Turatto, M., et al. 1992, ApJL, 387, L33

Scalzo, R., Aldering, G., Antilogus, P., et al. 2014, MNRAS, 440, 1498

Schlafly, E. F., \& Finkbeiner, D. P. 2011, ApJ, 737, 103

Schlegel, D. J., Finkbeiner, D. P., \& Davis, M. 1998, ApJ, 500, 525

Shappee, B. J., Piro, A. L., Holoien, T. W.-S., et al. 2016, ApJ, 826, 144

Shen, K. J., Bildsten, L., Kasen, D., \& Quataert, E. 2012, ApJ, 748, 35

Shen, K. J., Kasen, D., Miles, B. J., \& Townsley, D. M. 2018, ApJ, 854, 52

Silverman, J. M., Kong, J. J., \& Filippenko, A. V. 2012, MNRAS, 425, 1819

Silverman, J. M., Vinko, J., Kasliwal, M. M., et al. 2013, MNRAS, 436, 1225

Sim, S. A., Röpke, F. K., Hillebrandt, W., et al. 2010, ApJL, 714, L52

Smith, J. A., Tucker, D. L., Kent, S., et al. 2002, AJ, 123, 2121

Stetson, P. B. 1987, PASP, 99, 191

Stritzinger, M., Hamuy, M., Suntzeff, N. B., et al. 2002, AJ, 124, 2100

Stritzinger, M., \& Leibundgut, B. 2005, A\&A, 431, 423

Stritzinger, M., Suntzeff, N. B., Hamuy, M., et al. 2005, PASP, 117, 810

Stritzinger, M. D., Phillips, M. M., Boldt, L. N., et al. 2011, AJ, 142, 156

Suntzeff, N. B. 2000, in AIP Conf. Ser. 522, Cosmic Explosions, ed. S. S. Holt \& W. W. Zhang (Melville, NY: AIP), 65

Suntzeff, N. B. 2003, in Proc. ESO/MPA/MPE Workshop, From Twilight to

Highlight: The Physics of Supernovae, ed. W. Hillebrandt \& B. Leibundgut (Berlin: Springer), 183

Thomas, R. C., Branch, D., Baron, E., et al. 2004, ApJ, 601, 1019

Thompson, T. A. 2011, ApJ, 741, 82

Tinsley, B. M. 1979, ApJ, 229, 1046

Totani, T., Morokuma, T., Oda, T., et al. 2008, PASJ, 60, 1327

Tripp, R. 1998, A\&A, 331, 815

Turatto, M., Benetti, S., Cappellaro, E., et al. 1996, MNRAS, 283, 1

Wang, X., Filippenko, A. V., Ganeshalingam, M., et al. 2009, ApJL, 699, L139

Wang, X., Li, W., Filippenko, A. V., et al. 2009, ApJ, 697, 380

Woosley, S. E., \& Weaver, T. A. 1994, ApJ, 423, 371

Yamanaka, M., Maeda, K., Kawabata, M., et al. 2014, ApJL, 782, L35

Zhang, J.-J., Wang, X.-F., Bai, J.-M., et al. 2014, AJ, 148, 1

Zheng, W., Shivvers, I., Filippenko, A. V., et al. 2014, ApJL, 783, L24

Zheng, W., Silverman, J., Filippenko, A. V., et al. 2013, ApJ, 778L, $15 Z$ 